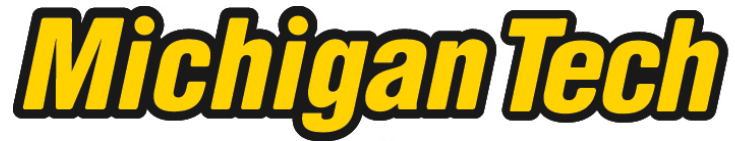 \\ Michigan Technological University Create the Future Digital Commons @ Michigan Tech
}

Dissertations, Master's Theses and Master's Reports - Open

Dissertations, Master's Theses and Master's

Reports

2013

Intergranular Corrosion and Stress Corrosion Cracking of Extruded AA6005A

David James Seguin

Michigan Technological University

Follow this and additional works at: https://digitalcommons.mtu.edu/etds

Part of the Materials Science and Engineering Commons

Copyright 2013 David James Seguin

\section{Recommended Citation}

Seguin, David James, "Intergranular Corrosion and Stress Corrosion Cracking of Extruded AA6005A", Dissertation, Michigan Technological University, 2013.

https://doi.org/10.37099/mtu.dc.etds/652

Follow this and additional works at: https://digitalcommons.mtu.edu/etds

Part of the Materials Science and Engineering Commons 


\title{
INTERGRANULAR CORROSION AND STRESS CORROSION CRACKING OF EXTRUDED AA6005A
}

\author{
By \\ Daniel James Seguin

\begin{abstract}
A DISSERTATION
Submitted in partial fulfillment of the requirements for the degree of DOCTOR OF PHILOSOPHY

In Materials Science and Engineering
\end{abstract}

MICHIGAN TECHNOLOGICAL UNIVERSITY

2013

(C) 2013 Daniel James Seguin 
This dissertation has been approved in partial fulfillment of the requirements for the Degree of DOCTOR OF PHILOSPHY in Materials Science and Engineering.

Department of Materials Science and Engineering

Dissertation Advisor: Calvin L. White.

Committee Member: $\quad$ Stephen Hackney

Committee Member: $\quad$ Mark Plichta

Committee Member: $\quad$ Richard Dickson

Department Chair: Stephen Kampe 


\section{Table of Contents}

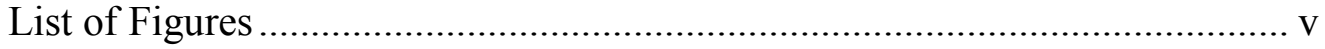

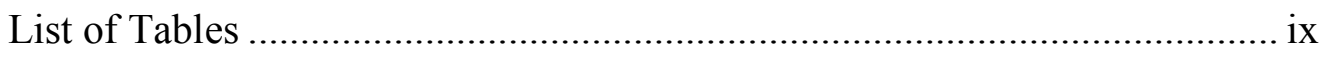

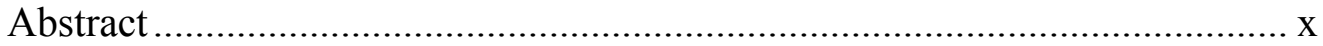

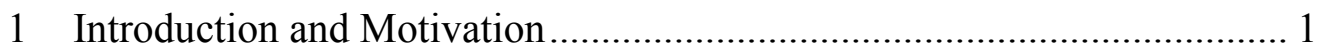

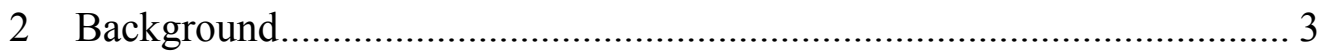

2.1 Aluminum Metallurgy …………………………………………….... 3

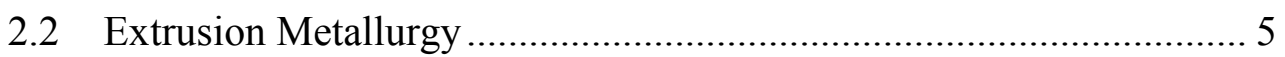

2.3 Corrosion of Aluminum and Aluminum Alloys ................................... 7

2.4 Stress Corrosion Cracking ……………………………………..... 18

3 Hypotheses and Literature Review....................................................... 25

3.1 IGC and SCC Susceptibility of AA6005A: Literature Review ........ 25

3.2 Mechanism of SCC in AA6005A: Literature Review …………….... 27

4 Experimental Methods and Results ………………..................................... 30

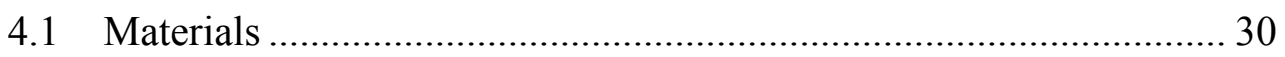

4.2 Electrochemical Behavior of AA6005A Extrusions............................ 31

4.2.1 Experimental Methods - Polarization Experiments ..................... 32

4.2.2 Results and Discussion - Polarization Experiments ................... 35

4.3 IGC of the Elongated Grain Structure in AA6005A …………........... 44

4.3.1 Experimental Methods - IGC of AA6005A ………………….... 44

4.3.2 Results and Discussion - IGC of AA6005A ………………........ 45 
4.3.3 Effects of Diffusion on IGC Velocity ....... 58

4.4 Qualitative measurement of SCC in AA6005A Extrusions ............... 76

4.4.1 Experimental Methods - Qualitative SCC of AA6005A ……...... 76

4.4.2 Results and Discussion - Qualitative SCC of AA6005A ............ 79

4.5 Quantitative measurement of SCC in AA6005A Extrusions ............. 84

4.5.1 Experimental Methods - Quantitative SCC of AA6005A ........... 84

4.5.2 Results - Quantitative SCC of AA6005A …………………........ 91

4.5.3 Discussion of Quantitative SCC Analysis.................................... 99

4.5.4 Effects of Stress Triaxiality and Local Electrochemical Reactions on Stress Corrosion Cracking of AA6005A-T6 …………………………….... 106

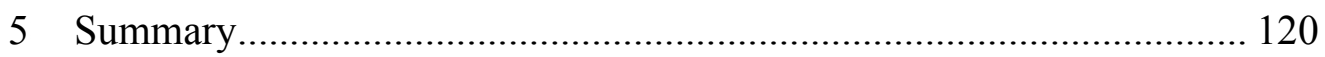

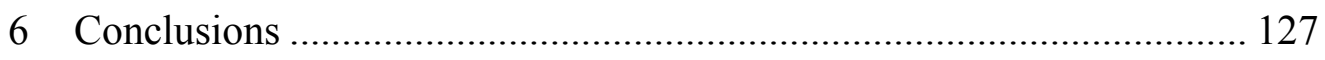

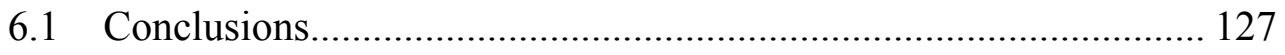

6.1.1 Conclusions based on Primary Hypotheses ................................ 127

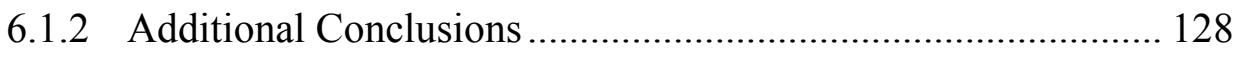

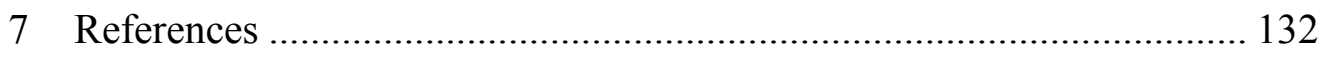

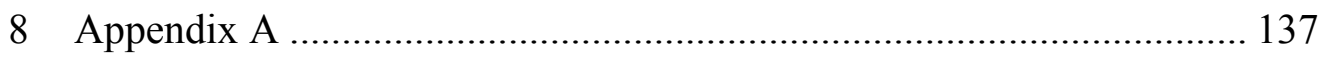

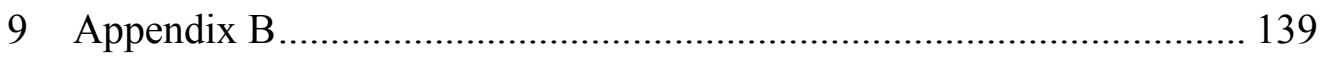




\section{List of Figures}

Figure 1:Typical grain structure of AA6005A extrusions ............................................ 6

Figure 2: Pourbaix diagram for pure aluminum ...................................................... 11

Figure 3: Schematic polarization curve with Tafel analysis...................................... 13

Figure 4: Method for determining anodic Tafel behavior. ........................................... 14

Figure 5: Effect of cathode to anode area ratio........................................................ 16

Figure 6: Schematic polarization curves for an active-passive metal......................... 17

Figure 7: Typical relationship between SC crack growth and stress intensity ........... 20

Figure 8: Schematic of a hydrogen embrittlement of SCC...................................... 22

Figure 9: Schematic of an anodic dissolution mechanism of SCC ............................. 23

Figure 10: Definition of coordinate system used to describe specimens ..................... 31

Figure 11: Sample configuration used in polarization experiments ............................. 33

Figure 12: Experimental setup for conducting polarization measurements................. 34

Figure 13: Effect of $\mathrm{pH}$ on the polarization behavior of AA6005A-T6 …................. 36

Figure 14:Effect of $\mathrm{pH}$ on the corrosion potential of AA6005A-T6 …...................... 37

Figure 15: Representative Tafel analysis for AA6005A-T6 ……………………….... 39

Figure 16: Effect of solution $\mathrm{pH}$ on the corrosion current density of AA6005A-T6 . 40

Figure 17: Effect of heat treatment on the polarization behavior of AA6005A ......... 41

Figure 18: Effect of a hydrogen recombination poison on the polarization behavior of

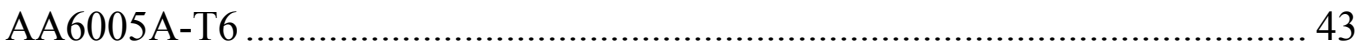


Figure 19: Effect of age treatment on the mode of corrosion attack ........................ 46

Figure 20: Core grain boundary microstructure of AA6005A-T4 .......................... 47

Figure 21: Core grain boundary microstructure of AA6005A-T6 ........................... 48

Figure 22: Core grain boundary microstructure of AA6005A-OA .......................... 49

Figure 23: Effect of $\mathrm{pH}$ on the severity of IGC in AA6005A-T6............................. 51

Figure 24: Typical anisotropic IGC attack observed in the elongated grain structure of AA6005A-T4

Figure 25: Maximum observed depth of IGC attack in the elongated grain structure for AA6005A-T4.

Figure 26: Maximum observed depth of IGC attack in the elongated grain structure for AA6005A-T6. 53

Figure 27: (200) X-Ray pole figure of elongated grain structure in AA6005A ......... 55

Figure 28: Three-dimensional geometric model used to describe the anisotropic corrosion attack in the elongated grain structure of AA6005A extrusions......... 56

Figure 29: Structure corrected corrosion path length for AA6005A-T6 ..................... 60

Figure 30: Structure corrected IGC velocity in AA6005A-T6 ............................... 61

Figure 31: Sample configuration used to qualitatively determine the variation in $\mathrm{pH}$ with corrosion depth 63

Figure 32: Variation in $\mathrm{pH}$ within a simulated corrosion path. 64

Figure 33: Sample configuration used to determine the local $\mathrm{pH}$ at varied IGC path lengths 66 
Figure 34: Effect of corrosion path length and acidity of bulk environment on the $\mathrm{pH}$ of the local environment within a simulated corrosion path 67

Figure 35: Model of IGC path used to determine IGC velocity ............................... 72

Figure 36: Test of diffusion-based model to predict IGC velocity in AA6005A-T6 . 74

Figure 37: Modified sub-sized tensile bar geometry used for BLT......................... 77

Figure 38: Experimental setup for conducting breaking load tests.......................... 78

Figure 39: System for conducting qualitative SCC analysis of AA6005A ............... 79

Figure 40: Effect of heat treatment on the residual strength of AA6005A................ 81

Figure 41: Effect of $\mathrm{pH}$ on SCC susceptibility in AA6005A-T6............................. 82

Figure 42: Effect of a hydrogen recombination poison on SCC susceptibility of AA6005A-T6 83

Figure 43: Sample geometry of CT specimens used for crack growth rate measurements 86

Figure 44: Modulus vs. crack length calibration of the sample configuration used in this work 88

Figure 45: Schematic of setup that was used for SCC-CGR experiments. 89

Figure 46: Results from CGR experiment of AA7475 sheet e .............................. 90

Figure 47: Crack growth rate of AA6005A-T6 extrusions..... 92

Figure 48: Typical SEM micgrograph of AA6005A-T6 fracture surface after conducting SCC testing. 93

Figure 49: SEM micrograph of fracture surface within elongated grain structure of

AA6005A-T6 94

vii 
Figure 50: Morphology of advancing crack in AA6005A-T6 96

Figure 51: Effect of a hydrogen recombination poison $\left(\mathrm{As}_{2} \mathrm{O}_{3}\right)$ on the crack growth rate of AA6005A-T6

Figure 52: Fracture surface of AA6005A-T6 CGR specimen exposed to a solution of $3.5 \% \mathrm{NaCl}+0.3 \% \mathrm{As}_{2} \mathrm{O}_{3}$ at $\mathrm{pH}=1.5$

Figure 53: Structure-corrected corrosion velocity with increasing corrosion depth along direction 1 for stressed and unstressed samples.

Figure 54: Effective corrosion path lengths along direction 2 for stressed SCC-CGR specimens with crack lengths greater than the $1 / 2$ thickness of the sample ..... 103

Figure 55: Calculated velocity profile along the crack front of a CT sample........... 105

Figure 56: Effect of plastic zone size on the stress state in a material.................... 107

Figure 57: Crack morphology at various depths in a compact tensile bar of AA6005A-T6

Figure 58: Schematic representation of the Nabarro-Herring model.

Figure 59: Local environment within a SC crack in a loaded CT specimen 114

Figure 60: Predicted polarization behavior at a stress corrosion crack tip for AA6005A-T6 exposed to $3.5 \% \mathrm{NaCl}$ at $\mathrm{pH}=1.50$.

Figure 61: Predicted polarization behavior at a stress corrosion crack tip for AA6005A-T6 exposed to $3.5 \% \mathrm{NaCl}$ at $\mathrm{pH}=2.0$.

Figure 62: Structure corrected corrosion velocity for loaded CT samples of AA6005A-T6 exposed to $3.5 \% \mathrm{NaCl}$ at $\mathrm{pH}=1.5$ or $\mathrm{pH}=2.0$ 119

viii 


\section{List of Tables}

Table 1: Primary alloying elements in wrought aluminum alloys ............................. 3

Table 2: Precipitation sequence for solutionized $6 \mathrm{xxx}$ alloys ................................. 4

Table 3: Composition specification for AA6005A alloy ....................................... 5

Table 4: Composition of AA6005A extrusions used in experiments ....................... 30

Table 5: Yield and tensile stresses for AA6005A extrusions with varied heat

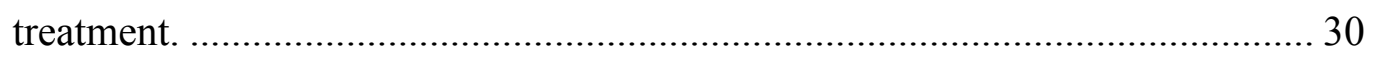

Table 6: Calculated corrosion path lengths along all directions for samples in a T4

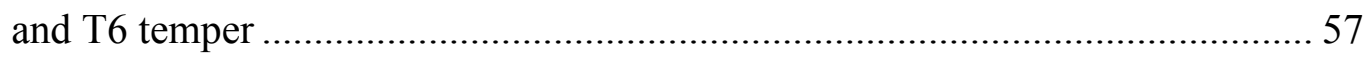

Table 7: Solubility products for several solid species that may control the $\mathrm{pH}$ within

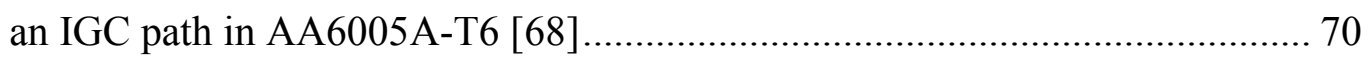

Table 8: Summary of electrochemical analysis for CT specimens exposed to $\mathrm{pH}=1.5$, and $\mathrm{pH}=2.0$ aerated saltwater solutions with and without an externally applied load. 


\section{Abstract}

A research program focused on understanding the intergranular corrosion (IGC) and stress corrosion cracking (SCC) behavior of AA6005A aluminum extrusions is presented in this dissertation. The relationship between IGC and SCC susceptibility and the mechanisms of SCC in AA6005A extrusions were studied by examining two primary hypotheses.

IGC susceptibility of the elongated grain structure in AA6005A exposed to low- $p H$ saltwater was found to depend primarily on the morphology of $\mathrm{Cu}$-containing precipitates adjacent to the grain boundaries in the elongated grain structure. IGC susceptibility was observed when a continuous (or semi-continuous) film of $\mathrm{Cu}$ containing phase was present along the grain boundaries. When this film coarsened to form discrete $\mathrm{Cu}$-rich precipitates, no IGC was observed. The morphology of the $\mathrm{Cu}$ rich phase depended on post-extrusion heat treatment.

The rate of IGC penetration in the elongated grain structure of AA6005A-T4 and AA6005A-T6 extrusions was found to be anisotropic with IGC propagating most rapidly along the extrusion direction, and least rapidly along the through thickness direction. A simple 3-dimensional geometric model of the elongated grain structure was accurately described the observed IGC anisotropy, therefore it was concluded that the anisotropic IGC susceptibility in the elongated grain structure was primarily due to geometric elongation of the grains. 
The velocity of IGC penetration along all directions in AA6005A-T6 decreased with exposure time. Characterization of the local environment within simulated corrosion paths revealed that a $\mathrm{pH}$ gradient existed between the tip of the IGC path and the external environment. Knowledge of the local environment within an IGC path allowed development of a simple model based on Fick's first law that considered diffusion of $A l^{3+}$ away from the tip of the IGC path. The predicted IGC velocity agreed well with the observed IGC velocity, therefore it was determined that diffusion of $\mathrm{Al}^{3+}$ was the primary factor in limiting IGC velocity.

The velocity of crack growth in compact tensile (CT) specimens of AA6005A-T6 extrusion exposed to $3.5 \% \mathrm{NaCl}$ at $p H=1.5$ was nearly constant over a range of applied stress intensities, exposure times, and crack lengths. The crack growth behavior oxf CT specimens of AA6005A-T6 extrusion exposed to a solution of $3.5 \% \mathrm{NaCl}$ at $p H=2.0$ exhibited similar behavior, but the crack velocity was $\sim 10.5 \mathrm{X}$ smaller than that those exposed to a solution at $p H=1.5$. Analysis of the local stress state and polarization behavior at the crack tip predicted that increasing the $p H$ of the bulk solution from 1.5 to 2.0 would decrease the corrosion current density at the crack tip by approximately $11.8 \mathrm{X}$. This predicted decrease in corrosion current density was in reasonable agreement with the observed decrease in SCC velocity associated with increasing the solution $p H$ from 1.5 to 2.0. The agreement between the predicted and observed SCC velocities suggested that the electrochemical reactions controlling SCC in AA6005A-T6 extrusions are ultimately controlled by the $p H$ gradient that exists between the crack tip and external environment. 


\section{Introduction and Motivation}

Extruded Aluminum is among the most versatile engineering materials available today. Its high strength-to-weight ratio, relatively low associated cost, formability, and recyclability have made aluminum alloys appealing materials for use in a broad range of applications. The ability to manufacture elongated sections with complex two-dimensional profiles makes extrusion an efficient processing route for structural components from aluminum alloys. Extruded aluminum alloys have been used as the primary construction material in many advanced structures including solar power plant frames and aerospace fuselages. To fully realize the range of potential applications of aluminum extrusions as structural components, it is essential to understand how the material will behave in corrosive service environments, such as an industrial marine environment. The research discussed in this dissertation is focused on understanding fundamental mechanisms of intergranular corrosion (IGC) and stress corrosion cracking (SCC) of extruded AA6005A alloy in simulated marine environments.

The original purpose of the research was to determine if IGC or SCC of AA6005A extrusions should be a major design consideration if there material were to be used in concentrated solar power plant (CSPP) frames in regions with a marine climate, such as Florida. The design lifetime for the CSPP frames was to be 30 years. 
The specifications of the CSPP frames indicated that the extruded members of AA6005A would see a maximum tensile load of approximately $44 \mathrm{MPa}\left(18 \% \sigma_{y}\right)$, and maximum compressive load of approximately $46 M P a$ in severe (hurricane) conditions. These design values served as a baseline for beginning the study of IGC and SCC of AA6005A, and were used to assess the viability of the material for use in the CSPP frames. 


\section{Background}

\subsection{Aluminum Metallurgy}

There are eight general classes of wrought aluminum alloys recognized by the Aluminum Association (AA). Each series is defined by primary alloying element(s) that are added to achieve desired properties of the final product (Table 1). Specific alloy designations within each class are defined by concentrations of primary and secondary alloying elements. The research presented here will focus on AA6005A extrusions. The primary alloying elements in AA6005A are $\mathrm{Mg}$ and $\mathrm{Si}$. There is limited literature available on intergranular corrosion (IGC) and no literature on stress-corrosion cracking (SCC) of AA6005A. Much of the background data relevant to the research program is therefore focused on other 6xxx alloys. Relevant comparisons to other alloy classes, such as $2 \mathrm{xxx}$ and $7 \mathrm{xxx}$, is also used to formulate hypotheses and propose research activities.

Table 1: Primary alloying elements in wrought aluminum alloys

\begin{tabular}{|c|c|c|c|c|c|c|c|c|}
\hline Series & $1 \mathrm{xxx}$ & $2 \mathrm{xxx}$ & $3 \mathrm{xxx}$ & $4 \mathrm{xxx}$ & $5 \mathrm{xxx}$ & $6 \mathrm{xxx}$ & $7 \mathrm{xxx}$ & $8 \mathrm{xxx}$ \\
\hline $\begin{array}{l}\text { Primary } \\
\text { Alloying } \\
\text { Elements }\end{array}$ & $\mathrm{N} / \mathrm{A}$ & $\mathrm{Cu}$ & $\mathrm{Mn}$ & $\mathrm{Si}$ & $\mathrm{Mg}$ & $\mathrm{Si}, \mathrm{Mg}$ & $\mathrm{Zn}$ & $\begin{array}{c}\text { Other } \\
(\mathrm{Li}, \\
\mathrm{Fe}, \\
\text { (etc.) }\end{array}$ \\
\hline
\end{tabular}

The combination of strength, formability, and good corrosion resistance make these 6xxx commonly used materials in engineering applications. Mechanical strength of solution treated $6 \mathrm{xxx}$ alloys can be improved by precipitation hardening 
(aging). Aging is generally done at intermediate temperatures $\left(\sim 185^{\circ} \mathrm{C}\right)$, but some degree of strengthening can be achieved by naturally aging the material for an extended period of time at room temperature. The primary strengthening phase of 6xxx alloys, $\mathrm{Mg}_{2} \mathrm{Si}(\beta)$, is formed through a sequence of steps, as shown in Table $2[1$, 2]:

Table 2: Precipitation sequence for solutionized 6xxx alloys

\begin{tabular}{|ll|l|}
\hline & & Precipitate Structure \\
\hline 1 & Saturated solid solution & FCC solid solution \\
\hline 2 & Solute rich Guinier Preston zones & Clusters of solute species \\
\hline 3 & Coherent $\beta$ ' $\left(\mathrm{Mg}_{5} \mathrm{Si}_{6}\right)$ precipitates & Monoclinic \\
\hline 4 & Semi-coherent $\beta$ ' precipitates & Structure unknown \\
\hline 5 & Incoherent $\beta\left(\mathrm{Mg}_{2} \mathrm{Si}\right)$ precipitates & Cubic \\
\hline
\end{tabular}

The first step in the precipitation sequence for 6xxx alloys is formation of small Guinier Preston (GP) zones. The GP zones combine to form coherent monoclinic $\mathrm{Mg}_{5} \mathrm{Si}_{6}\left(\beta{ }^{\prime \prime}\right)$ precipitates upon thermal treatment. Further thermal treatment causes the $\beta$ " phase to form semi-coherent $\beta$ ' precipitates. The structure and chemistry of $\beta$ ' is not well known, but it has been suggested that $\beta$ ' has a hexagonal structure, and forms rod-like precipitates [3]. The final step in the precipitation sequence involves the formation of $\beta$, which is incoherent and has a cubic crystal structure.

Optimal strength of 6xxx alloys is achieved with a dense, homogeneous network of fine $\beta$ " precipitates in the material [1]. Adding $M g$ and $S i$ in a near 5:6 molar ratio and using a well-controlled heat treatment process can promote precipitation of $\beta$ ". The alloy AA6005A incorporates this $\mathrm{Mg}: \mathrm{Si}$ molar ratio to yield a 
near optimal medium strength wrought aluminum alloy. The AA composition specification for AA6005A alloy is given in Table 3. Because of this near-optimized composition, extruded AA6005A alloy is more easily formed and less quench sensitive than some of the other 6xxx alloys with similar mechanical strength specifications (e.g. AA6061). In addition to $M g$ and $S i$, the AA specification for AA6005A allows for up to $0.3 w t . \% C u$. The $C u$ is added to improve the mechanical strength of the alloy through precipitation of certain strengthening phases. However, it has been demonstrated through a number of reports that $C u$ in excess of about 0.12 $w t . \%$ can make the alloy susceptible to IGC when the alloy has certain thermomechancial histories [4-8].

Table 3: Composition specification for AA6005A alloy

\begin{tabular}{|c|c|c|c|c|c|c|c|c|l|l|}
\hline$C r$ & $C u$ & $F e$ & $M g$ & $M n$ & $S i$ & $T i$ & $Z n$ & $\begin{array}{l}\text { Other, } \\
\text { each }\end{array}$ & $\begin{array}{l}\text { Other, } \\
\text { total }\end{array}$ & $A l$ \\
\hline$\leq 0.30$ & $\leq 0.30$ & $\leq 0.35$ & $\begin{array}{c}0.40- \\
0.70\end{array}$ & $\leq 0.50$ & $\begin{array}{c}0.50- \\
0.90\end{array}$ & $\leq 0.10$ & $\leq 0.20$ & $\leq 0.05$ & $\leq 0.15$ & Bal. \\
\hline
\end{tabular}

\subsection{Extrusion Metallurgy}

Aluminum extrusion is a high-temperature deformation process in which elongated parts with a fixed cross sectional profile are manufactured by pressing a pre-heated billet through a precision die. This seemingly simple process is actually quite complex because extrusion is a non-steady-state process in which deformation mechanisms, heat generation, and friction evolve each extrusion cycle. There are several features characteristic of extruded profiles that are pertinent to the present study. 
Extrusions of AA6005A alloy, as with many other extruded alloys, have a duplex grain structure consisting of an elongated grain structure in the core of the material, and an equiaxed layer near the surface as illustrated in Figure 1. The elongated structure in air quenched AA6005A contains fully recrystallized grains that are oriented along the extrusion direction. The equiaxed layer consists of randomly oriented grains which generally have higher angle boundaries than those found in the elongated core structure [9]. A thick recrystallized layer is known to be detrimental to the mechanical performance of the final product [10]. Studies have also shown that an equiaxed grain structure may be more susceptible to IGC than an elongated structure in extruded $\mathrm{Al}$ alloys [9]. Limiting the degree of deformation during extrusion, and achieving a rapid quench after extrusion can minimize the thickness of the equiaxed layer [11].

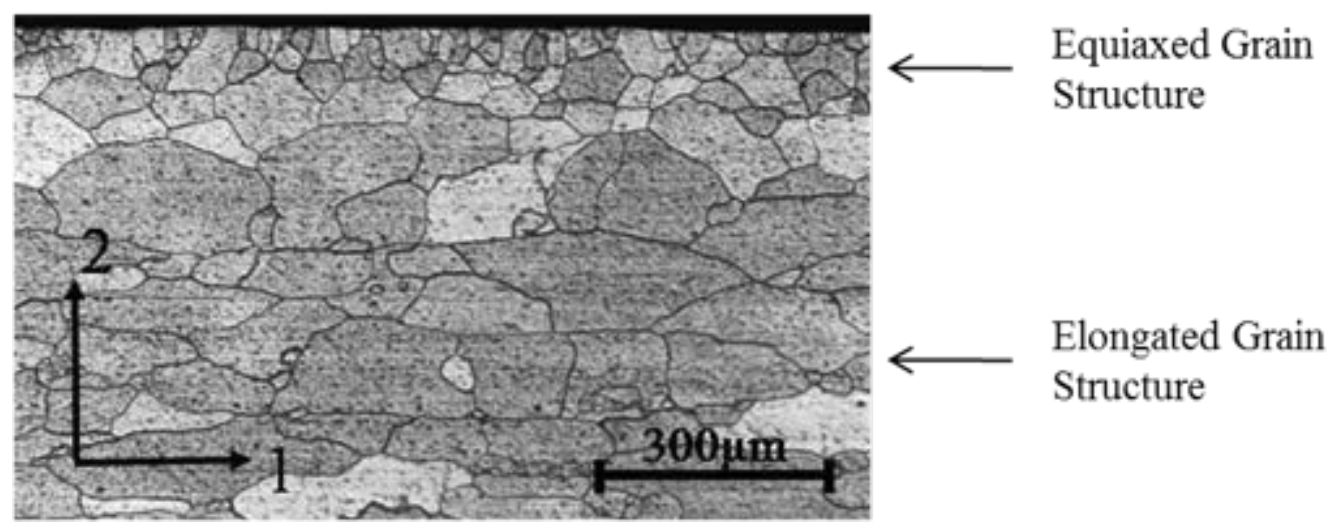

Figure 1:Typical grain structure of AA6005A extrusions consisting of equiaxed grains near the extrusion surface (top of micrograph), and elongated grains (near bottom of micrograph) in the core of the extrusion. Direction " 1 " is the extrusion direction, and direction " 2 " is the through thickness direction. 
The elongated grain structure in aluminum extrusions can give rise to anisotropic mechanical properties and anisotropic corrosion behavior $[11,12]$. The origin of the anisotropic corrosion behavior in the elongated structure of wrought aluminum alloys has been extensively reported [12-16]. Researchers have determined that texture and grain boundary misorientation angle can have a dramatic effect on the grain boundary precipitate structure in wrought aluminum alloys, thereby giving rise to anisotropic IGC $[9,12]$.

In many cases, anisotropic IGC has been simply attributed to geometric anisotropy of the grains providing a less tortuous path for corrosion attack along the deformation direction [15-17]. Zhang et al. studied the relationship between grain elongation and preferential attack along the extrusion direction in AA2024-T3 plate [15]. Zhang modeled the anisotropic grain structure as a two-dimensional brick wall with the edge lengths of the bricks corresponding to the nominal grain size along each direction. Zhang quantitatively predict the anisotropic corrosion attack in the anisotropic grain structure using this simple geometric configuration, and considering the density of initiation sites along different surfaces in the material. Several studies have used models similar to Zhang's to describe anisotropic corrosion attack in wrought aluminum alloys [12, 14-17].

\subsection{Corrosion of Aluminum and Aluminum Alloys}

Corrosion is the degradation of a material due to electrochemical interactions with the service environment. There are two fundamental chemical reactions that 
control all corrosion processes in metallic systems. The first is the anodic (oxidation) reaction in which metal ions are dissociated from the parent alloy, leaving behind free electrons (Equation 1). Electrons created by the anodic reaction are conducted through the metal to participate in the cathodic (reduction) reaction, sometimes at a significant distance from the location of the anodic reaction. The cathodic reaction involves species from the environment (typically ionic) consuming the electrons that are produced at the site of the anodic reaction. The most common cathodic reaction in acidic environments involves reduction of $\mathrm{H}^{+}$ions to form $\mathrm{H}_{2}$ gas (Equation 2) [18]. The electrochemical driving force for corrosion reactions is the difference in the halfcell potentials $(E)$ for the anodic and cathodic reactions. The standard state half-cell potentials for each of the reactions are given below.

Equation 1 [19]

$$
A l=A l^{3+}+3 e^{-} \quad E=-1.66 V_{S H E}
$$

Equation 2 [19]

$$
2 H^{+}+2 e^{-}=H_{2} \quad E=0 V_{S H E}
$$

The $H_{2}$ evolution reaction (Equation 2) occurs in three distinct steps [18]. First, $\mathrm{H}^{+}$reacts with an electron from the metal surface to form an adsorbed $H$ atom $\left(H_{a d s}\right)$ at the metal surface (Equation 3). The $H_{a d s}$ then combines with another $H_{a d s}$ to form an adsorbed hydrogen molecule $\left(H_{2 a d s}\right)$ (Equation 4). The $H_{2 a d s}$ molecules then combine to form an $\mathrm{H}_{2}$ bubble at the metal surface. Adding a hydrogen recombination poison, such as $A s^{3+}$, to the electrolyte tends to inhibit the recombination of $H_{a d s}$, thereby increasing the concentration of atomic $H$ in the material [20,21]. 
Equation 3 [18]

$$
H^{+}+e^{-}=H_{a d s}
$$

Equation 4 [18]

$$
2 H_{a d s}=H_{2 a d s}
$$

The effects of temperature and concentration of ionic species on the half-cell corrosion potentials of the reactions listed above can be determined using the Nernst equation (Equation 5) [18, 19]. The standard form of the Nernst equation is:

\section{Equation 5}

$$
E=E^{o}-\frac{R T}{n F} \ln \frac{a_{Y}^{y} a_{Z}^{z} \cdots}{a_{D}^{d} a_{E}^{e} \cdots}
$$

Where $E$ is the half-cell corrosion potential for a reaction, $E^{o}$ is the half-cell corrosion potential for a reaction when all reactants and products are in their standard states, $R$ is the ideal gas constant, $T$ is the absolute temperature of the reaction, $n$ is the number of electrons involved in the half cell reaction, $F$ is Faraday's constant $(96,500 \mathrm{C} / \mathrm{mol})$, and $a_{X}{ }^{x}$ is the activity of specific species involved in the half cell reaction. The activities of the products of the reaction are in the numerator of in Equation 5, and the activities of the reactants are in the denominator. The activity of a dissolved species is usually assumed to be equal to its concentration, and the activity of a pure solid or gas at atmospheric pressure is unity. The exponents in Equation 5 are the stoichiometric coefficients of the corresponding elements in the half-cell reactions.

Aluminum is an electrochemically active metal, but is generally regarded as having good corrosion resistance due to the rapid formation of a passive oxide film on the metal surface. The passive film consists of two layers: 1) A continuous 
amorphous oxide film adjacent to the parent metal and 2) A porous crystalline layer between the amorphous layer and the external environment [22]. The passive layer, which has the approximate composition of $\mathrm{Al}_{2} \mathrm{O}_{3}$, acts as a kinetic barrier that slows dissolution of the corroding material. Discontinuities in the $\mathrm{Al}_{2} \mathrm{O}_{3}$ film often serve as initiation sites for localized corrosion in $A l$ alloys [23].

A Pourbaix diagram illustrates the range of $\mathrm{pH}$ and electrochemical potential in which an $\mathrm{Al}_{2} \mathrm{O}_{3}$ passive layer is thermodynamically stable on pure aluminum (Figure 2). It is evident from the Pourbaix diagram that the passive layer on aluminum becomes unstable in acidic or alkaline environments when at higher applied potential [19]. The range of $p H$ and potential in which the passive layer is stable depends on the concentration of the corrosion product (either $\mathrm{Al}^{3+}$ or $\mathrm{AlO}_{2}^{-}$) in solution. The values of $\mathrm{pH}$ at which given concentrations of $\mathrm{Al}^{3+}$ and $\mathrm{AlO}_{2}^{-}$are in equilibrium with the passive layer are determined using the Nernst equation. The vertical lines located on the upper left side of the Pourbaix diagram correspond to conditions in which $\mathrm{Al}_{2} \mathrm{O}_{3}$ and $\mathrm{Al}^{3+}$ are in thermodynamic equilibrium. These vertical lines are key features of the Pourbaix diagram that will be used to develop a model to describe IGC penetration later in the dissertation. 


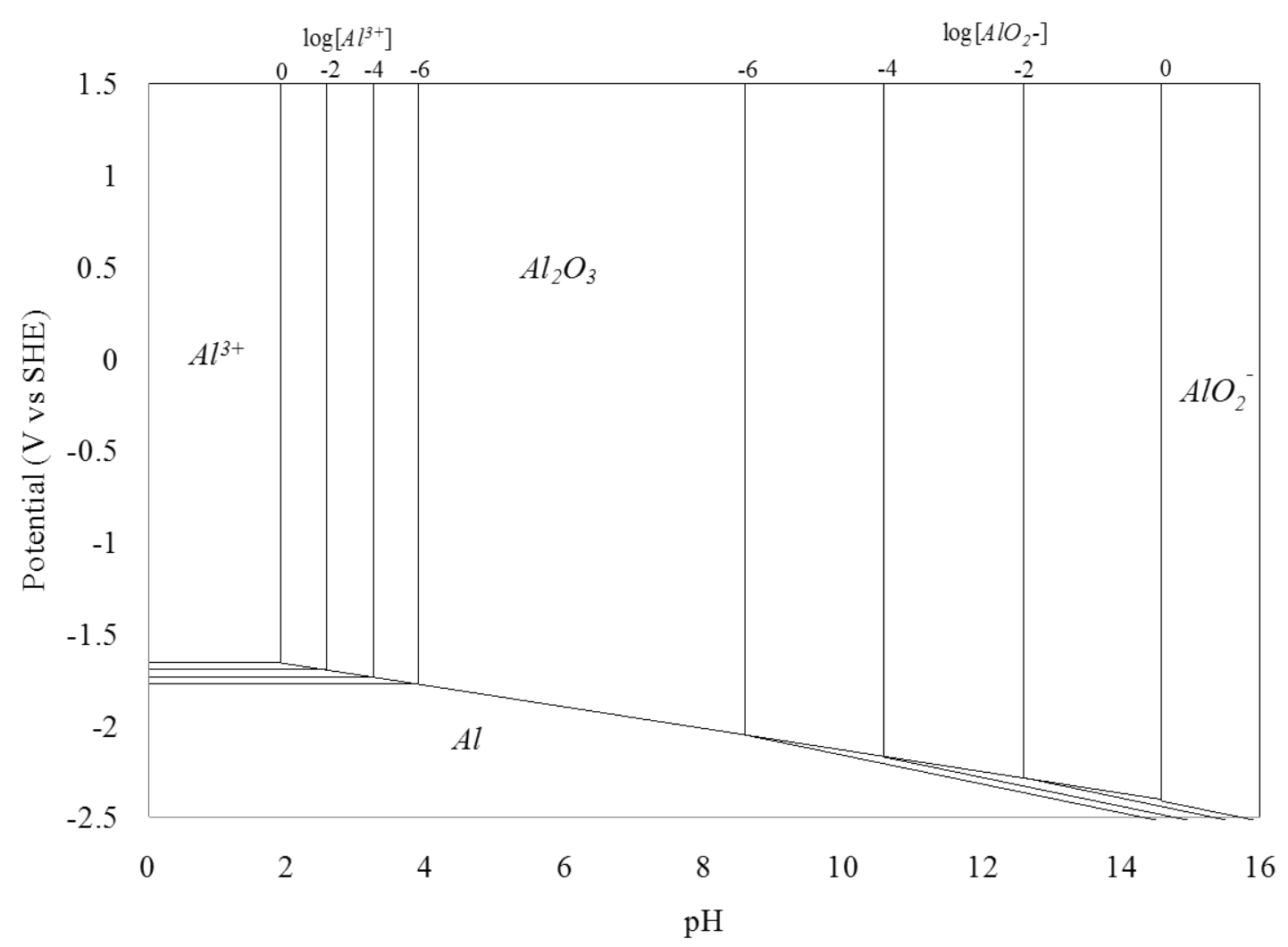

Figure 2: Pourbaix diagram for pure aluminum. The stability of the $\mathrm{Al}_{2} \mathrm{O}_{3}$ depends on the applied potential, $\mathrm{pH}$, and the concentration of corrosion product.

The corrosion rate and corrosion potential of a material in specific environments may be characterized by conducting polarization measurements. Polarization measurements are performed by applying a range of known electrochemical potentials to a sample and measuring the resulting corrosion current density. The output of a polarization measurement is a single curve that contains information about both the cathodic and anodic reactions involved in a specific corrosion reaction. It is important to note that the observed polarization curves actually reflect the electrochemical response of the electrolytic solution adjacent to the surface of the sample. 
The polarization behavior of a corrosion reaction depends not only on the equilibrium electrochemical properties of the anodic and cathodic reactions, but also on kinetic properties of the reactions. The exchange current density $\left(i_{o}\right)$ of a half-cell reaction is a characteristic value that defines the kinetics of that reaction under equilibrium conditions. Changes in ionic concentration and temperature can shift the value of $i_{o}$ to higher or lower values, thereby altering the observed polarization behavior of a corrosion reaction. The kinetics of a reaction may also depend on both the surface properties of the alloy. For example, the rate of cathodic and anodic reactions may be slowed by the presence of a passive layer on the surface of the corroding metal due to poor electrical conductivity of the passive layer [24]. This would tend to shift the exchange current density of the cathodic reaction to a lower value, thereby changing the observed values of corrosion potential $\left(E_{C o r r}\right)$ and corrosion current density $\left(i_{C o r r}\right)$.

Values of corrosion current density are extracted from polarization curves by performing Tafel analysis [18, 19, 25]. A simple example of a Tafel analysis is illustrated in Figure 3. The solid line illustrates the overall polarization response of the material, and the dashed lines illustrate the Tafel curves for the anodic and cathodic reactions. Tafel analysis involves extrapolating a region of linear $V$ vs. $\log (i)$ behavior in the anodic and cathodic portions of the polarization curve. When the slope of the $V$ vs. $\log (i)$ is near linear, the reaction rate is controlled by activation polarization. Activation polarization occurs when the observed polarization behavior of the electrochemical reaction reflects the activation energy for electron transfer 
between the electrolyte and electrode (sample) [19]. The slope of each of the curves is the Tafel constant, $\beta$. The intersection of the anodic and cathodic Tafel curves corresponds to the corrosion current density, $i_{\text {Corr }}$, for a given corrosion reaction.

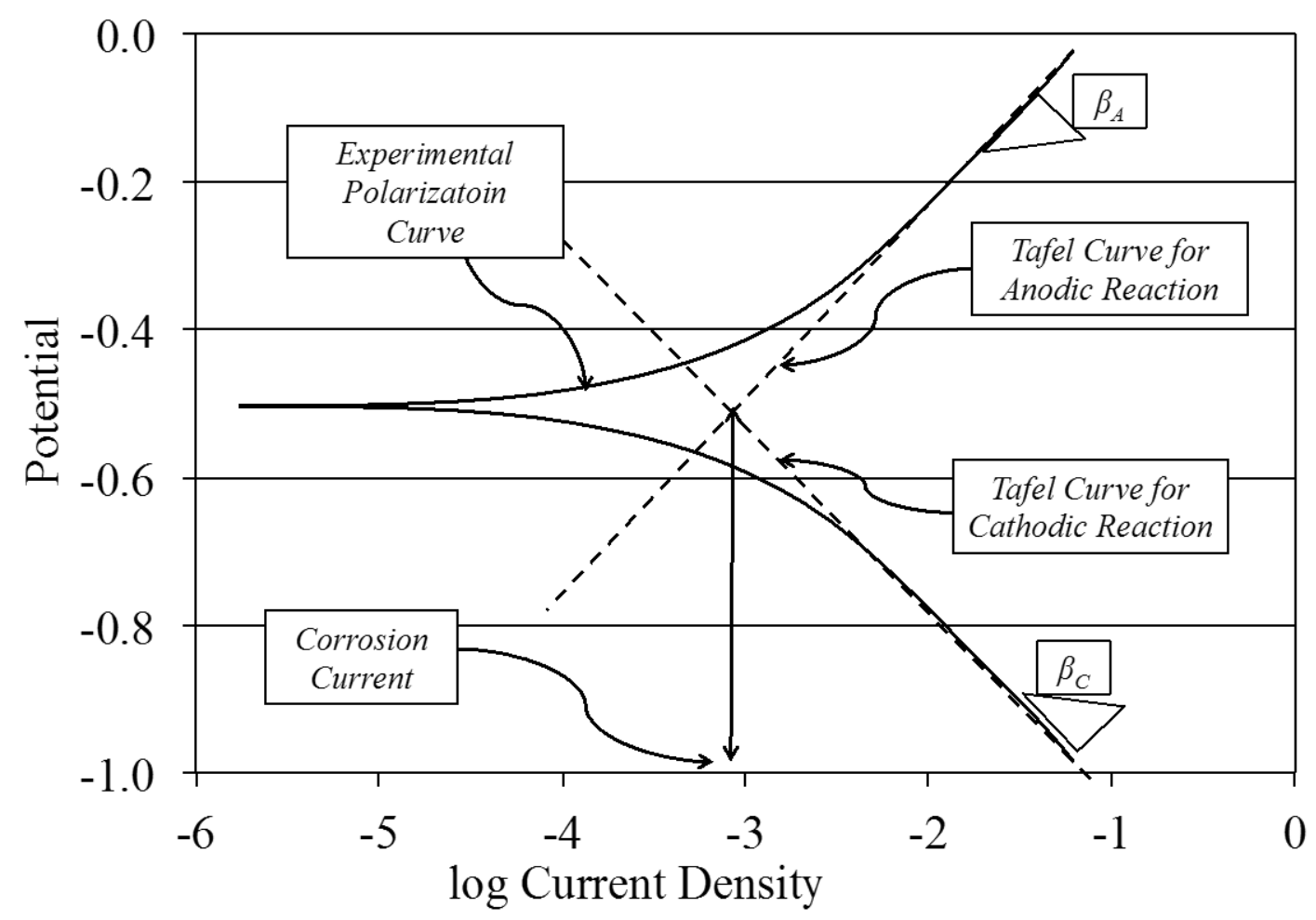

Figure 3: Schematic polarization curve with Tafel analysis.

In practice, Tafel analysis of the anodic portion of a polarization curve is often more difficult than the situation illustrated in Figure 3 because there is rarely a significant linear region in the anodic portion of the $V$ vs. $\log (i)$ curve. A technique summarized by Jones can be used to perform Tafel analysis of the anodic portion of a polarization curve [19]. Jones' technique is performed by first determining a region of Tafel behavior in the cathodic portion of a polarization curve. A series of points at 
values of electrochemical potential above the region of linearity in the cathodic curve (indicated by hollow circles in Figure 4) are then selected. The values of corrosion current density at these points $\left(i_{E x p}\right)$ are subtracted from values of corrosion current density on the cathodic Tafel curve $\left(i_{C}\right)$ to determine the anodic corrosion current density $\left(i_{A}\right.$, indicated by the filled circles in Figure 4$)$ at values of electrochemical potential below the open circuit corrosion potential. An anodic Tafel curve is then generated by extrapolating the calculated values of $i_{A}$ to higher electrochemical potentials.

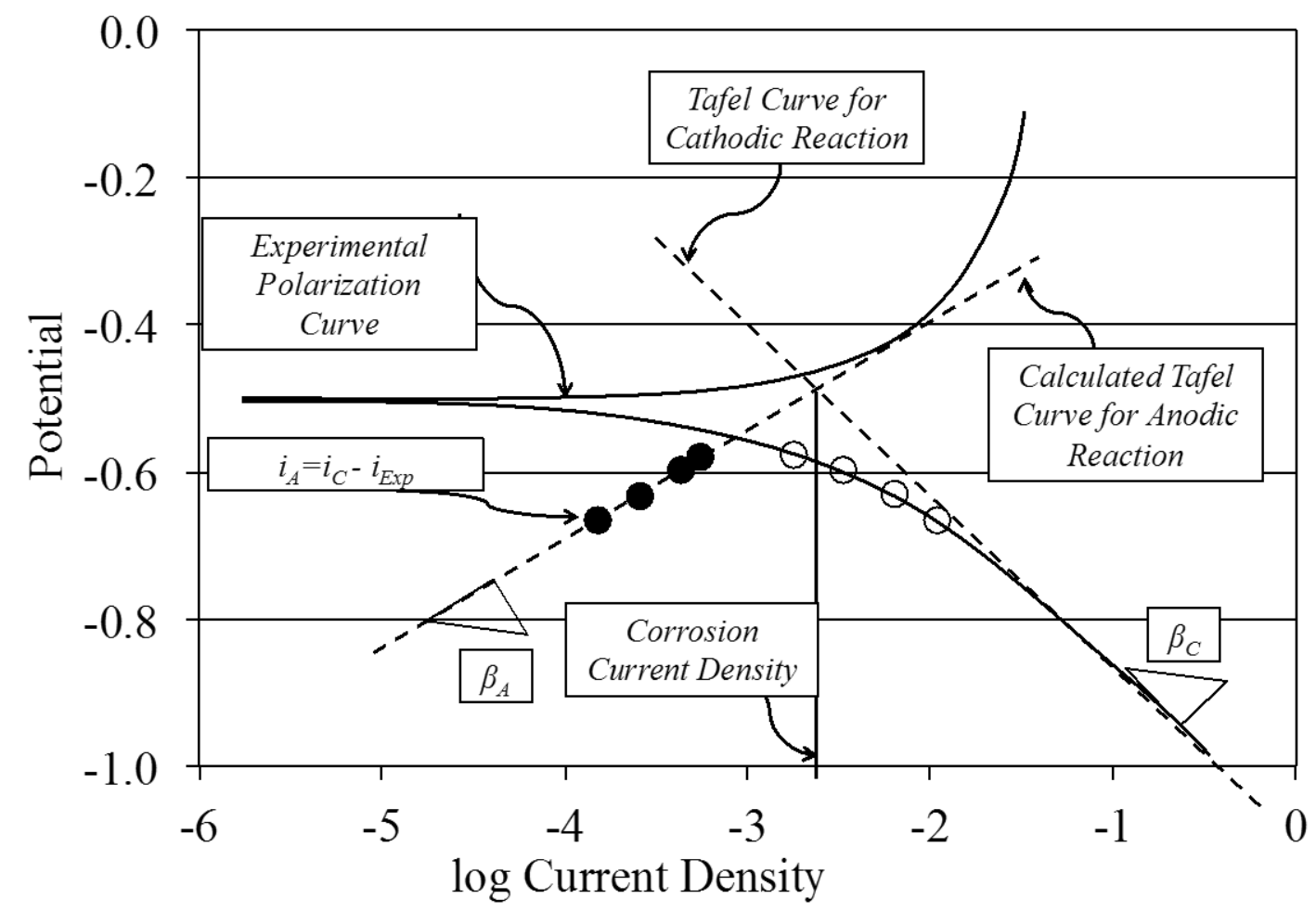

Figure 4: Method for determining anodic Tafel behavior.

The relative surface areas on which anodic and cathodic reactions are occurring can have a significant influence on the overall kinetics of a corrosion 
reaction [19]. This concept is schematically illustrated with Tafel plots in Figure 5. First consider a corrosion reaction in which the cathode to anode area ratio is $1: 1$. Assume the exchange current density of the anodic reaction is $i_{O A}$, and the exchange current density of the cathodic reaction is $i_{o C l}$ in this case. The resulting corrosion current density in this case would be $i_{\text {Corr }}$. Now consider a case in which the cathodic surface area is increased by $100 \mathrm{X}$ and the anodic surface area is held constant. Increasing the cathodic surface area would increase the exchange current density of the cathodic reaction, causing the cathodic Tafel curve to intersect the anodic Tafel curve at a higher value of current density and corrosion potential. The resulting corrosion current density would therefore be increased to $i_{\text {Corr } 2}$. 


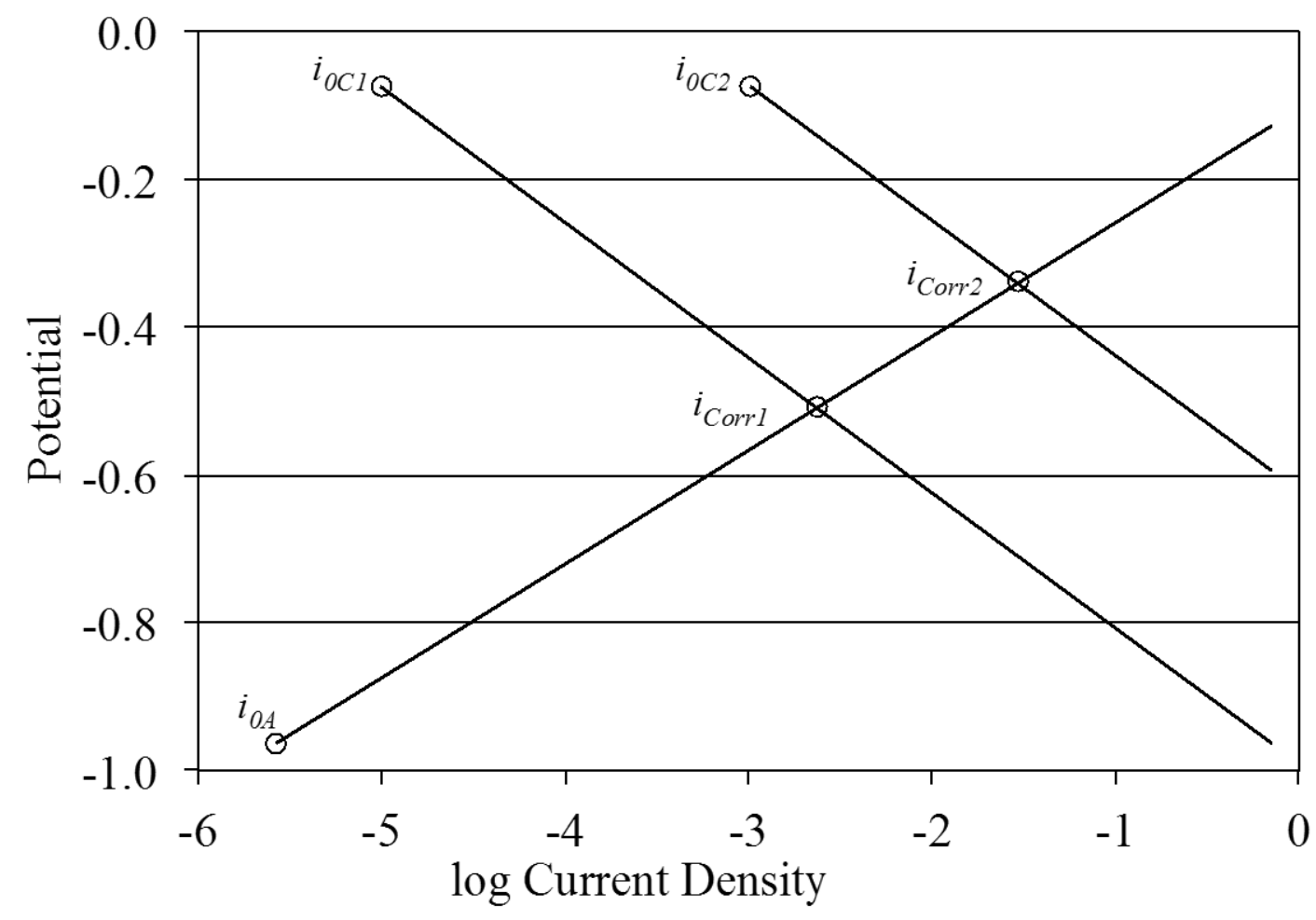

Figure 5: Effect of cathode to anode area ratio on the kinetics of a corrosion reaction.

Schematic anodic and cathodic polarization curves for a metal exhibiting active-passive behavior (e.g. aluminum) are illustrated as dashed lines in Figure 6. The experimentally observed current density (solid black lines) of the alloy is the algebraic sum of the cathodic and anodic polarization current, represented by dashed lines in Figure 6. The regions of active, passive, and transpassive behavior are indicated in Figure 6A. The point at which the anodic and cathodic reactions intersect corresponds to the characteristic corrosion rate (measured corrosion current density, $\left.i_{\text {Corr }}\right)$ and open circuit corrosion potential, $E_{\text {Corr }}$, of the reaction. If the cathodic polarization curve intersects the anodic polarization curve reaction at a potential 
corresponding to passive behavior (as shown in Figure 6A), the corrosion rate is limited by the presence of a passive film, and is relatively low. If the cathodic reaction is shifted such that it intersects the transpassive region in the anodic polarization curve, as shown in Figure 6B, the passive layer is not stable, and the resulting corrosion rate is larger. A cathodic reaction in an acidic environment can be shifted up or down by changing the $\mathrm{pH}$ of the solution, or changing the partial pressure of gases involved in the cathodic reaction [18].

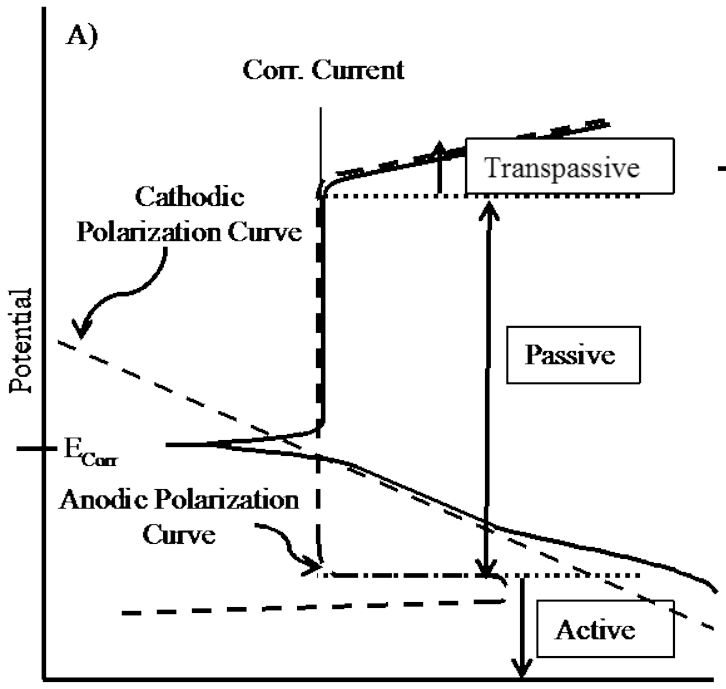

Log Current Density

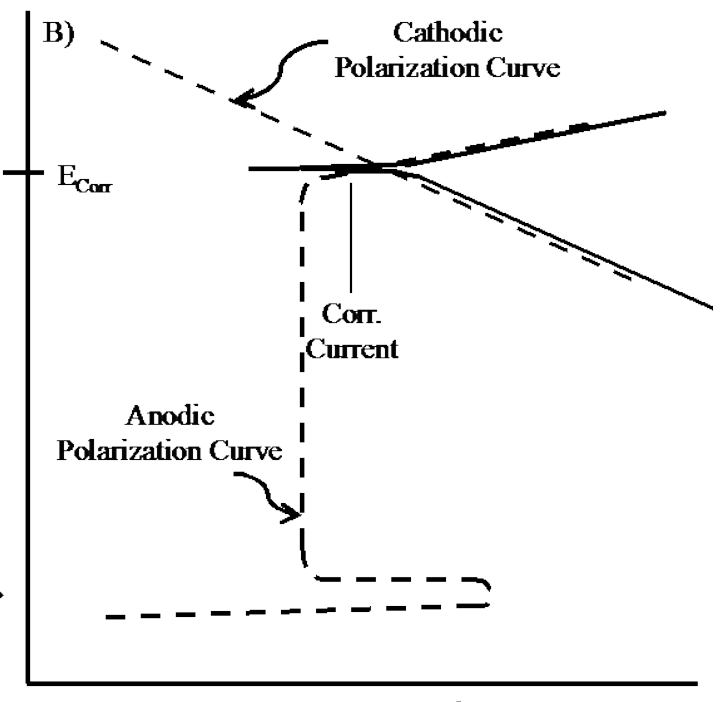

Log Current Density

Figure 6: Schematic polarization curves for a metal exhibiting active-passive behavior. The dashed lines illustrate the cathodic and anodic reactions, and the bold solid lines illustrate the observed polarization behavior. If the cathodic and anodic reactions intersect at a potential corresponding to passive behavior (as shown in Figure 6A), the corrosion rate (current) is much lower than if the cathodic and anodic reactions intersect at a potential corresponding to transpassive behavior (Figure 6B). 
Galvanic corrosion occurs when dissimilar metals are electrically coupled and exposed to a corrosive environment. Galvanic coupling can cause the corrosion current and corrosion potential to vary significantly from the values of the uncoupled metals. The degree of this shift in corrosion behavior depends on the exchange current densities, the slope of polarization curves, and the corrosion potential of the coupled metals. In general, the metal that has a lower corrosion potential in a given environment will act as the anode in the galvanic reaction, and will be preferentially corroded. Conversely, the metal which has a higher corrosion potential provides a surface on which the cathodic reaction can occur, and will be protected [18, 19, 25].

Galvanic corrosion can occur on a microscopic scale in multi-phase alloys due to differences in the corrosion potentials of their microconstituents. Intergranular corrosion commonly occurs along grain boundaries due to microgalvanic coupling between grain boundary precipitates and precipitate free zone (PFZ) $[18,19,23,26]$. The PFZ is a solute-depleted layer adjacent to the grain boundary. Propagation of IGC generally requires that the corrosion potential between the grain boundary precipitate and PFZ be greater than $100 \mathrm{mV}$, and that the grain boundary precipitates contributing to IGC be continuous along the grain boundary [26].

\subsection{Stress Corrosion Cracking}

Stress corrosion cracking (SCC) is a slow crack growth (typically $<10^{-6} \mathrm{~m} / \mathrm{s}$ ) phenomenon that occurs when a material is loaded in tension and exposed to a specific corrosive environment. SCC is a primary engineering concern, as it generally results in brittle failure of a nominally ductile material at relatively low applied 
stresses. SCC failure often occurs at tensile loads well below the yield strength of the bulk material. The tensile stresses may be externally applied or they may be residual stresses from the manufacturing process. Because cracks are often not visible, and there is rarely macroscopic evidence of mechanical deformation of the bulk material, SCC failures often occur without warning. Stress corrosion cracking is broadly associated with materials that demonstrate active-passive corrosion behavior, and SCC failure generally occurs when a material at an electrochemical potential near the transition between active and passive behavior or between passive and transpassive behavior [27].

Growth of SCC cracks is often divided into three distinct regimes: a) Crack initiation and stage I crack growth, b) Steady-state (Stage II) crack growth, and c) Stage III crack growth and Catastrophic failure [19, 27]. Figure 3 schematically depicts the evolution of SCC crack growth with increasing stress intensity. Experimental distinction of the three regimes can be difficult because there is a slow and continuous transition between them. SCC initiation occurs when the stress intensity reaches a critical value, $K_{I S C C}$. The value of $K_{I S C C}$ is dependent upon both the material and service environment. SCC initiation sites may be preexisting defects in the material (surface scratch, inclusion, intermetallic phase, etc), or they can be generated by exposure to a corrosive environment during service (breach in passive film, corrosion pits, etc.). Final SCC failure occurs when the crack has grown to a length in which the applied stress intensity exceeds the critical stress intensity, $K_{I C}$, for the remaining ligament. [18, 19, 28, 29]. 


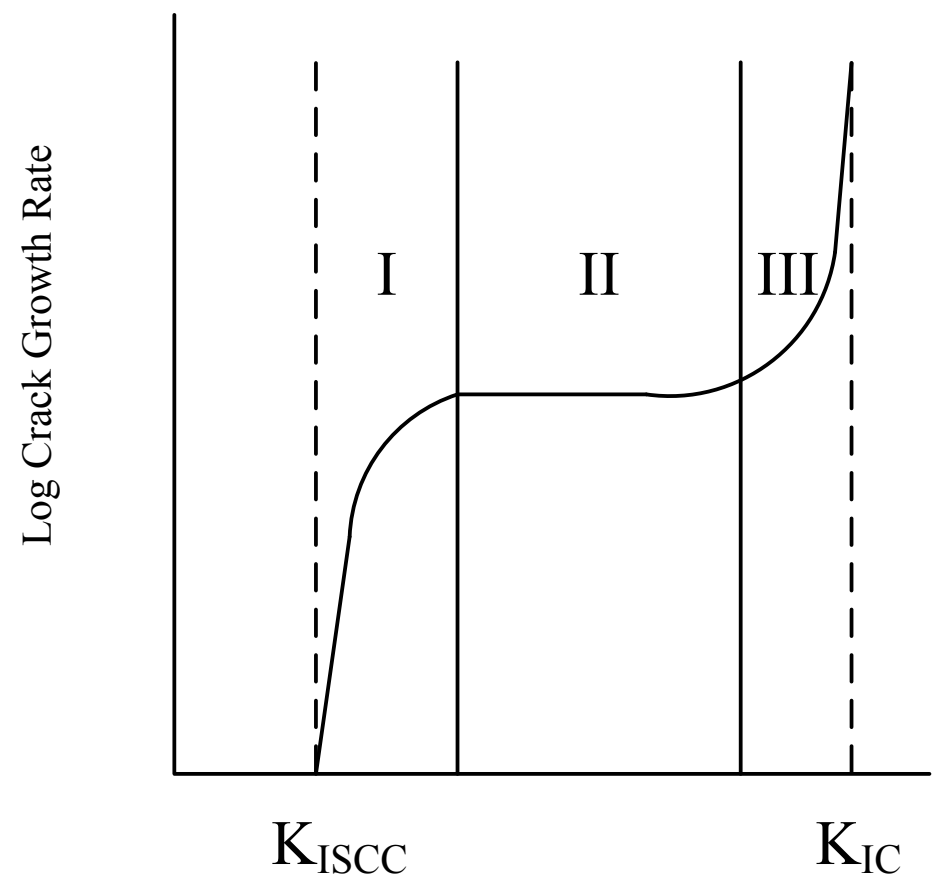

Stress Intensity

Figure 7: Typical relationship between SC crack growth and stress intensity. The three regimes of SCC are indicated.

Stress corrosion cracks are fractographically characterized as having sharp, branched crack tips that may be either transgranular or intergranular $[19,25,30,31]$. There is rarely evidence of corrosion products on the fracture surfaces of SCC failures. The underlying mechanisms for SCC are not yet fully understood. There are two general classes of mechanisms that have been proposed to explain SCC in various material systems $[19,27]$.

Some researchers argue that SCC is essentially a hydrogen embrittlement phenomenon [19, 27, 32-35]. There are two general models that have been proposed 
to explain hydrogen embrittlement; hydrogen enhanced decohesion (HED), and hydrogen enhanced localized plasticity (HELP). According to the HED model, atomic hydrogen accumulates at "trapping sites" such as dislocations or cracks and causes a localized weakening of the lattice $[33,34,36]$. According to the HELP model enhanced plasticity occurs at the crack tip due to absorbed atomic $H$ [32]. Some researchers have attempted to test a hydrogen embrittlement mechanism qualitatively by adding a hydrogen recombination poison (HRP) to the test environment $[14,21$, 37]. A hydrogen recombination poison slows the recombination of $H_{a d s}$ (Equation 4), thereby increasing the concentration of atomic $H$ in the material. The increased concentration of atomic $H$ in the material tends to magnify the effects hydrogen embrittlement. The $A s^{3+}$ ion is a commonly used HRP. Small amounts of $A s^{3+}$ can be added to the environment by dissolving $\mathrm{As}_{2} \mathrm{O}_{3}$ into the test solution. The solubility limit of $\mathrm{As}_{2} \mathrm{O}_{3}$ in water is $\sim 20 \mathrm{~g} / \mathrm{L}$, though only a small concentration $(0.3 \mathrm{~g} / \mathrm{L})$ is necessary to magnify the effects of hydrogen embrittlement. 


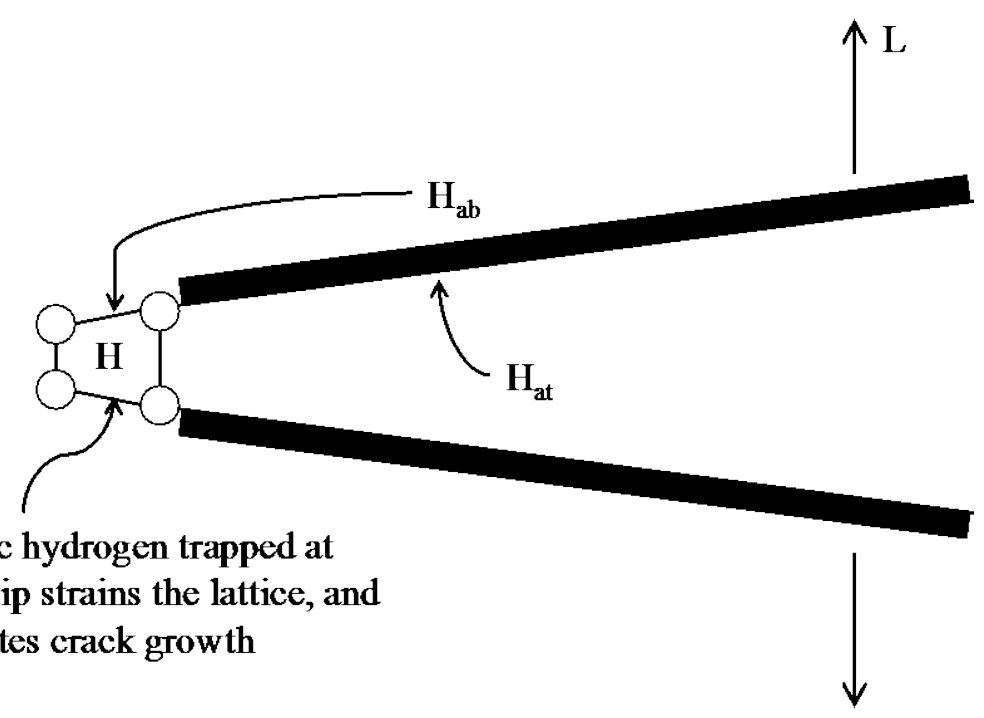

Figure 8: Schematic of a hydrogen embrittlement of SCC. Atomic hydrogen generated by the cathodic reaction is absorbed, and migrates to the crack tip thus facilitating crack growth by hydrogen embrittlement.

Other researchers argue that SCC is a highly localized dissolution phenomenon caused by a discontinuity in the passive film at a crack tip $[19,30,35]$ (Figure 9). In dissolution-based models of SCC, the passive film at the crack tip ruptures with applied stress, allowing rapid dissolution of the freshly exposed material. The dissolution rate at the crack tip is related to local electrochemical properties of the material and environment. 


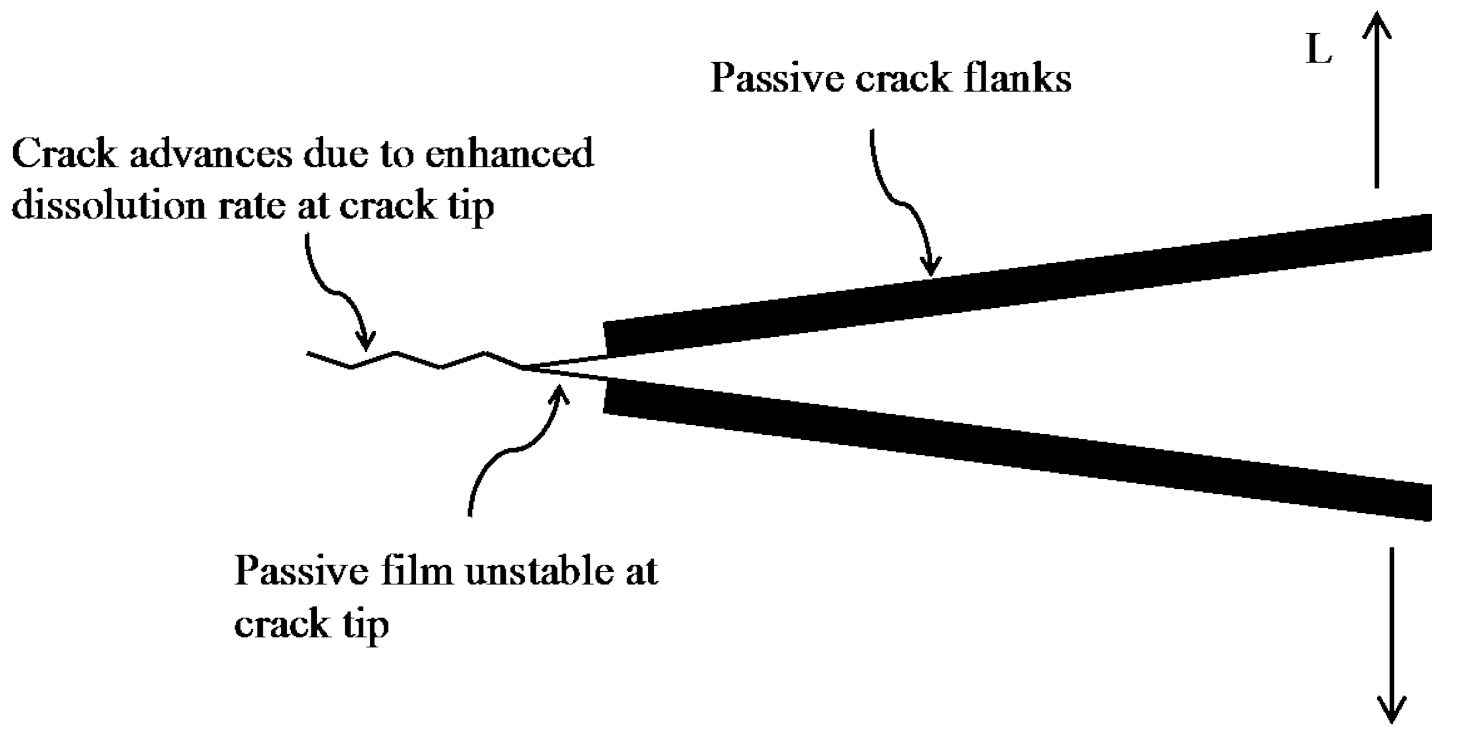

Figure 9: Schematic of an anodic dissolution mechanism of SCC. The passive film becomes unstable at the crack tip, thereby facilitating crack advance due to enhanced dissolution at the crack tip.

The local environment within a stress-corrosion crack often behaves as an isolated system, and may be much different from that surrounding environment. For example, when 7075 aluminum alloy is exposed to an aqueous salt water environment, the $p H$ near the crack tip remains relatively constant at $p H \sim 3.5$ when the applied stress intensity factor is greater than $K_{I S C C}$, regardless of the $\mathrm{pH}$ of exterior environment [38-41]. Some researchers have argued that the isolated nature of the crack tip environment lowers the concentration of dissolved $\mathrm{O}_{2}$, thereby reducing the local $p H$ if the bulk solution is near neutral [42]. Others have observed that the $p H$ within a stress-corrosion crack in an $\mathrm{Al}$ alloy is controlled by thermodynamic equilibrium between $\mathrm{Al}^{3+}$ and a solid species within the system (such as $\mathrm{Al}(\mathrm{OH})_{3}$ or $\left.\mathrm{Al}_{2} \mathrm{O}_{3}\right)[39,40,43,44]$. A gradient in electrical potential has also been observed 
between the electrolyte near the tip of SC cracks and the electrolyte in the bulk environment. [45]. The gradient in potential has been attributed to a difference in chemical potential between the active crack tip and the passive crack flanks with applied stress. The difference in potential between the crack tip and crack flank is thought to lead to enhanced dissolution at the crack tip, or a buildup of atomic $H$ at the crack tip. There do not appear to have been any studies on the local environment within stress corrosion cracks of AA6005A.

Although a significant volume of research has been devoted to developing and proving a single explanation for SCC, there has been no universal acceptance of any single mechanism to explain SCC to date $[30,31]$. Such an investigation was beyond the scope of the research proposed here, though experiments designed to clarify the mechanism of SCC in AA6005A extrusions were performed. 


\section{Hypotheses and Literature Review}

There are two primary hypotheses on which this research program was based. These hypotheses are:

1) Metallurgical and environmental conditions that promote IGC susceptibility in the elongated grain structure of AA6005A extrusions, will also promote SCC susceptibility.

2) The mechanism of SCC in AA6005A extrusions is enhanced anodic dissolution at the crack tip due to combined effects of applied load and local electrochemical reactions at the crack tip.

\subsection{IGC and SCC Susceptibility of AA6005A: Literature Review}

In aluminum alloys, SCC is an intergranular phenomenon [27]. Furthermore, conditions (material and environmental) that promote IGC have been shown to promote SCC in a number of different aluminum alloys. Liu et al. observed a continuous increase in IGC penetration rate with increasing tensile stress in underaged AA2024 when exposed to a neutral saltwater environment $[13,46]$. In other work, Dymek demonstrated that SCC and IGC resistances in AA2519 alloy are simultaneously improved by cold working and decreasing the copper content to promote a more uniform distribution of precipitates [47]. This direct relationship between IGC and SCC has also been observed in 5xxx and 7xxx alloys [31, 48-51]. These observations suggest that metallurgical and environmental conditions that promote IGC in AA6005A extrusions will also promote SCC, in accordance with 
hypothesis 1 . Though the IGC behavior of 6xxx alloys has been well documented [48, 52-54], no study linking IGC and SCC susceptibility in AA6005A exists to date.

Nisanciaglu and coworkers conducted a series of studies on the IGC behavior of unstressed $\mathrm{AlMgSi}(\mathrm{Cu})$ extrusions with compositions similar to AA6005A and containing between 0.02 and $0.2 \mathrm{wt} . \% \mathrm{Cu}$ [4-8]. These studies investigated the effects of $\mathrm{Cu}$ content and thermal history on the corrosion behavior of the equiaxed grain structure at the extruded surfaces. It was determined that the equiaxed grain structure becomes susceptible to IGC if the material contains more than $\approx 0.12 \mathrm{wt} \% \mathrm{Cu}$, is in an underaged condition, and is slow quenched from extrusion temperature. The authors suggest that this increased susceptibility to IGC is due to microgalvanic coupling between the Q-phase precipitates $\left(\mathrm{Al}_{4} \mathrm{Mg}_{8} \mathrm{Si}_{7} \mathrm{Cu} 2\right)$ and the PFZ along grain boundaries. The Q-phase, which has a higher corrosion potential than the aluminum matrix, was found to form a semi-continuous film along the grain boundaries in the equiaxed grain structure when the material was in an underaged condition. Artificial aging coarsened the $\mathrm{Cu}$-rich film to form discrete Q-phase precipitates, which these authors suggest interrupts the cathodic path thus improving IGC resistance of the material.

No study on the IGC behavior of the elongated grain structure of at the core of AA6005A extrusions exists to date. Press quenched extrusions of AA6005A contain approximately $95 \%$ elongated grain structure by volume (Figure 1). It is therefore necessary to conduct a study on the IGC behavior of the elongated grain structure in order to test the first hypothesis. 


\subsection{Mechanism of SCC in AA6005A: Literature Review}

Burliegh published a broad review of studies on SCC of wrought aluminum alloys [30]. A dissolution mechanism was generally favored to explain SCC in 2xxx alloys, whereas, whereas a hydrogen embrittlement mechanism was favored for SCC of 7xxx alloys. The second hypothesis that was examined by this research was anodic dissolution aggravated by applied stress is the mechanism of SCC in AA6005A extrusions.

Those supporting a dissolution mechanism in 2xxx alloys have observed that grain boundary dissolution will occur with or without applied stress, but that the dissolution is accelerated by applied stress [55] or applied anodic overpotential [56]. Susceptibility to IGC and SCC in 2xxx alloys is generally attributed to microgalvanic coupling between $\mathrm{Cu}$-containing grain boundary precipitates (GBPs) and the $\mathrm{Al}$ matrix. The GBPs in this case have a higher corrosion potential than the $A l$ matrix, thereby act as the cathode in the microgalvanic reaction $[13,30,46,47,57,58]$. It is known that AA6005A extrusions with elevated copper content have demonstrated IGC susceptibility due to the presence of GBPs that are cathodic relative to the PFZ [4-8]. It is thus possible that SCC in AA6005A extrusions will be analogous to $2 \mathrm{xxx}$ alloys due to these similarities in grain boundary chemistry and corrosion behavior.

Though the mechanism of SCC in AA6005A is hypothesized to involve anodic dissolution, it is necessary to also consider the possibility that a hydrogen embrittlement (HE) mechanism may contribute to SCC in AA6005A. Numerous studies have been published which studied the effects of ageing treatment and alloy 
composition HE susceptibility of 7xxx alloys [59-62]. Knight found that decreased copper content and underaging tended to increase the open circuit corrosion potential near a stress corrosion crack tip in AA7075 and AA7079. These conditions were also found to increase stage II crack velocity. This behavior was attributed to a decrease in copper content within GBPs, thereby rendering the GBPs more anodic relative to the PFZ. In this case, the GBP was found to act as the anode and the PFZ as the cathode in the corrosion reaction. It was argued that this increase in driving force for the corrosion reaction accelerated hydrogen production, thereby increasing the likelihood that atomic hydrogen may be absorbed into the alloy [62]. In general, susceptibility to SCC in 7xxx alloys has been attributed to copper replacing some of the zinc in $\mathrm{MgZn}_{2}$ in GBPs. The presence of the copper tends to increase the corrosion potential of the GBP to a level more similar to that of the PFZ, thereby decreasing the thermodynamic driving force for the electrochemical reactions governing SCC.

It is noteworthy that in the case of $7 \mathrm{xxx}$ alloys that thought to be susceptible to SCC by a HE mechanism, the grain boundary precipitates are active relative to the PFZ, and act as the anode in the microgalvanic reaction. Conversely, in the case of 2xxx alloys, which are susceptible to SCC by an anodic dissolution mechanism, the grain boundary precipitates are noble relative to the PFZ, and serve as a cathode in the microgalvanic reaction. From an electrochemical standpoint, extrusions of the alloy AA6005A are known to have grain boundary microstructure similar to that of 2xxx alloys. This similarity in electrochemical behavior at the grain boundaries served as the basis for formulated the second hypothesis. 
There do not appear to have been any studies published on the mechanisms of SCC in AA6005A. Therefore experiments designed to test both anodic dissolution and hydrogen embrittlement phenomena were carried out to determine the fundamental mechanism of SCC in AA6005A. 


\section{Experimental Methods and Results}

\subsection{Materials}

Sections of AA6005A extrusion were obtained from a commercial vendor.

The nominal composition of the material used in the experiments is given in Table 4. Note that the elevated $C u$ content is in the range where IGC susceptibility would be expected based on the observations of the Nisanciaglu group [4-8]. The extrusions were received in an as-extruded state (i.e. no post-extrusion thermal treatment). The sample thickness was $3.175 \mathrm{~mm}$ for all materials used in this study. The extrusions were air quenched, and the reduction ratio was $44: 1$.

Table 4: Composition of AA6005A extrusions used in experiments. All values are in weight percent.

\begin{tabular}{|c|c|c|c|c|c|c|c|c|}
\hline $\mathrm{Si}$ & $\mathrm{Fe}$ & $\mathrm{Cu}$ & $\mathrm{Mn}$ & $\mathrm{Mg}$ & $\mathrm{Ti}$ & $\mathrm{Zn}$ & $\mathrm{Ni}$ & $\mathrm{Al}$ \\
\hline 0.590 & 0.195 & 0.178 & 0.115 & 0.434 & 0.033 & 0.000 & 0.005 & Balance \\
\hline
\end{tabular}

Samples cut from the extrusions were aged in a laboratory furnace to a T4 ( 0 $h r$ at $\left.185^{\circ} \mathrm{C}\right)$, T6 $\left(6 h r\right.$ at $\left.185^{\circ} \mathrm{C}\right)$, or OA $\left(24 h r\right.$ at $\left.185^{\circ} \mathrm{C}\right)$. The T6 temper tempering procedure was found to give maximum hardness for the material tested. The average yield and tensile stresses for each of the tempers investigated are given in Table 5.

Table 5: Yield and tensile stresses for AA6005A extrusions with varied heat treatment.

\begin{tabular}{|c|c|c|}
\hline Age Treatment & Yield Stress $(\boldsymbol{M P a})$ & Tensile Strength $(\mathbf{M P a})$ \\
\hline T4 & 155 & 205 \\
\hline T6 & 255 & 300 \\
\hline OA & 250 & 300 \\
\hline
\end{tabular}


The coordinate system used to describe the different directions in the material is illustrated in Figure 10. Direction 1 corresponds to the extrusion direction, direction $\mathbf{2}$ corresponds to the through thickness direction, and direction $\mathbf{3}$ corresponds to the transverse direction.

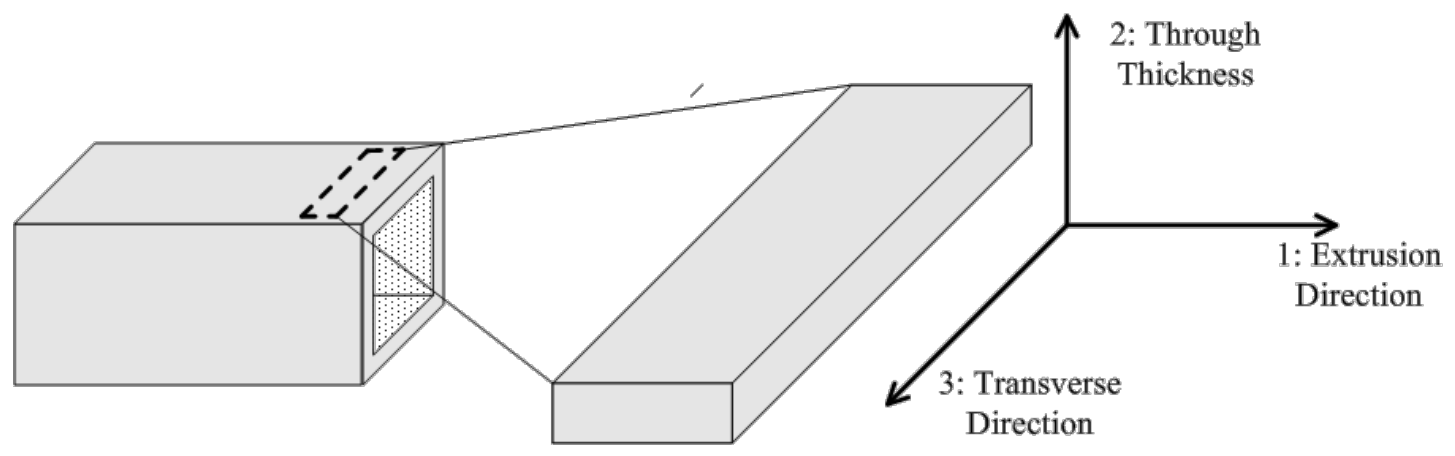

Figure 10: Definition of coordinate system used to describe specimens. Directions 1, $\mathbf{2}$, and 3 correspond to the extrusion direction, through thickness direction, and transverse direction, respectively.

\subsection{Electrochemical Behavior of AA6005A Extrusions}

The first step in characterizing the corrosion behavior of the elongated grain structure in AA6005A extrusions was to conduct experiments designed to understand the effects of environment and heat treatment on the bulk electrochemical behavior of the alloy. It is known that the local environment within an occluded region in an IGC or SCC path may differ significantly from that of the external environment. The experiments described in this section were therefore designed to give insight to the electrochemical behavior of within the occluded regions of the alloy as well as understand the bulk electrochemical behavior of the material. The results from these 
experiments served as a basis for drawing conclusions from IGC and SCC experiments.

\subsubsection{Experimental Methods - Polarization Experiments}

The effects of heat treatment and environment on the electrochemical behavior of AA6005A extrusions were studied by conducting polarization measurements using an EG\&G potentiostat/galavnostat. Specimens were aged to a $\mathrm{T} 4, \mathrm{~T} 6$ or OA condition prior to preparation. The specimens were machined into $1 \mathrm{~cm}$ $\times 1 \mathrm{~cm}$ squares using a $\mathrm{CNC}$ end mill. A small ribbon of stainless steel wire was attached to each specimen using conductive silver paste. The stainless steel wire served as an electrical lead to the specimen in the polarization experiments, and did not contact the electrolyte during the experiments. The prepared specimens were mounted in two-part epoxy, and the $1 \mathrm{~cm}^{2}$ exposed surface was mechanically ground to a $600 \mathrm{~g}$ finish in 4 steps to fully remove the equiaxed grain structure. The specimen configuration is illustrated in Figure 11. 


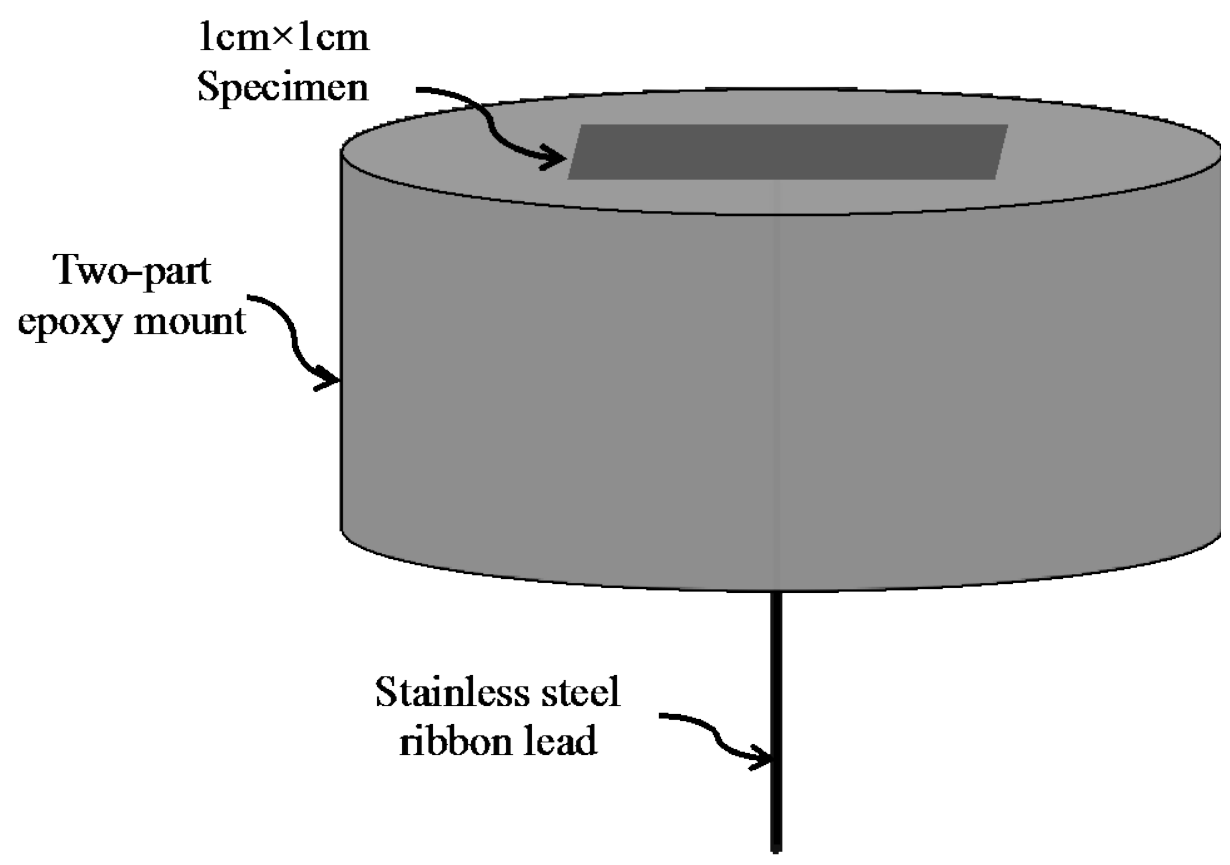

Figure 11: Sample configuration used in polarization experiments for AA6005A extrusions.

The experimental setup for the polarization measurements is schematically illustrated in Figure 12. The prepared specimens were placed in the aqueous test solution, and a $3 \mathrm{~mm}$ diameter luggin probe was placed near the specimen surface. The luggin probe senses the potential of the solution in the vicinity of the sample, and the potentiostat allows the sample to be held at known values of electrochemical potential during the polarization experiments. A platinum counter electrode with a surface area of $2 \mathrm{~cm}^{2}$ was placed in the test solution near the sample. A saturated calomel reference electrode (SCE) was immersed in solution of saturated $\mathrm{KCl}$ in a separate container. A salt bridge containing saturated $\mathrm{KCl}$ solution was used to connect the luggin to the SCE reference electrode. The potential between the specimen and the SCE was scanned from $-2.0 \mathrm{~V}$ to $0.5 \mathrm{~V}$ vs. SCE to yield both 
cathodic and anodic segments of the polarization curve. The current density of the corrosion reaction $(i)$ was measured between the counter electrode and the specimen, and was plotted against the applied the potential ( $V$ vs. SCE).

Once the experiment was appropriately assembled, the system was allowed to equilibrate for 180 seconds prior to beginning the polarization measurements. Upon reaching equilibration, the specimens were held at the starting potential (-2 V vs. $S C E$ ) for 30 seconds. The potential was then scanned in a stepwise manner. The step size was $1 \mathrm{mV}$ and the dwell time was $0.1 \mathrm{~s}$.

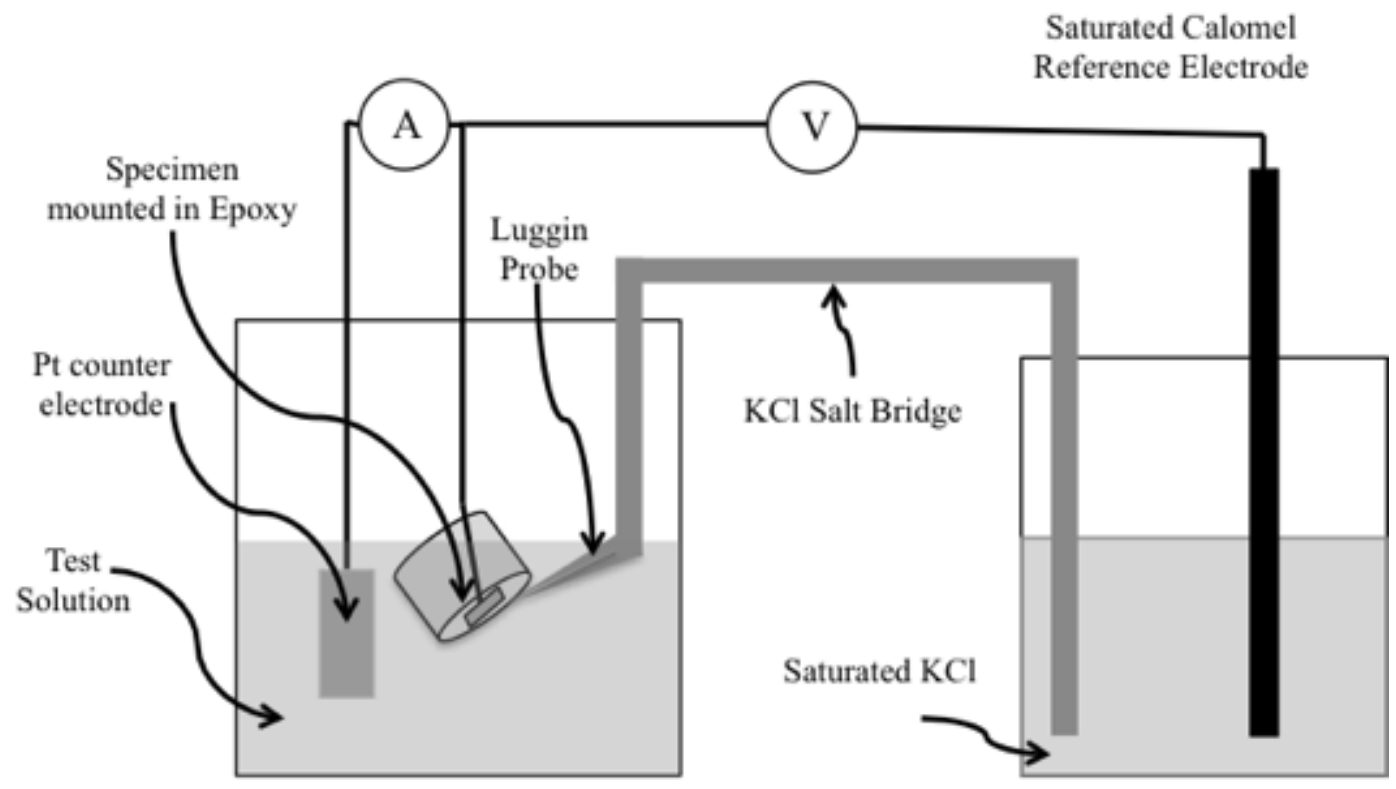

Figure 12: Experimental setup for conducting polarization measurements on AA6005A extrusions

The polarization experiments were conducted in $3.5 \% \mathrm{NaCl}$ solutions with $1.20<p H<5.50$. The $p H$ was adjusted by adding $H C l$ to the solutions. The $p H$ of the solution without $\mathrm{HCl}$ addition was approximately 5.5. A small amount $(0.3 \mathrm{~g} / \mathrm{L})$ of 
$\mathrm{As}_{2} \mathrm{O}_{3}$ was added to the solution for some experiments to study the effects of a hydrogen recombination poison on the polarization behavior of AA6005A-T6 extrusions.

\subsubsection{Results and Discussion-Polarization Experiments}

The effect of $p H$ on the anodic polarization behavior of AA6005A-T6

exposed to an aqueous solution of $3.5 \% \mathrm{NaCl}$ is illustrated in Figure 13. The stability of the passive layer on AA6005A-T6 depended on $p H$ of the test solution, which is consistent with what would be expected from the Pourbaix diagram for $A l$ (Figure 2). The material exhibited passive behavior over a large range of applied potential when the solution was in the range $3.00 \leq p H \leq 5.50$. No notable range of passive behavior was exhibited when the solution was at $p H \leq 2.00$. 


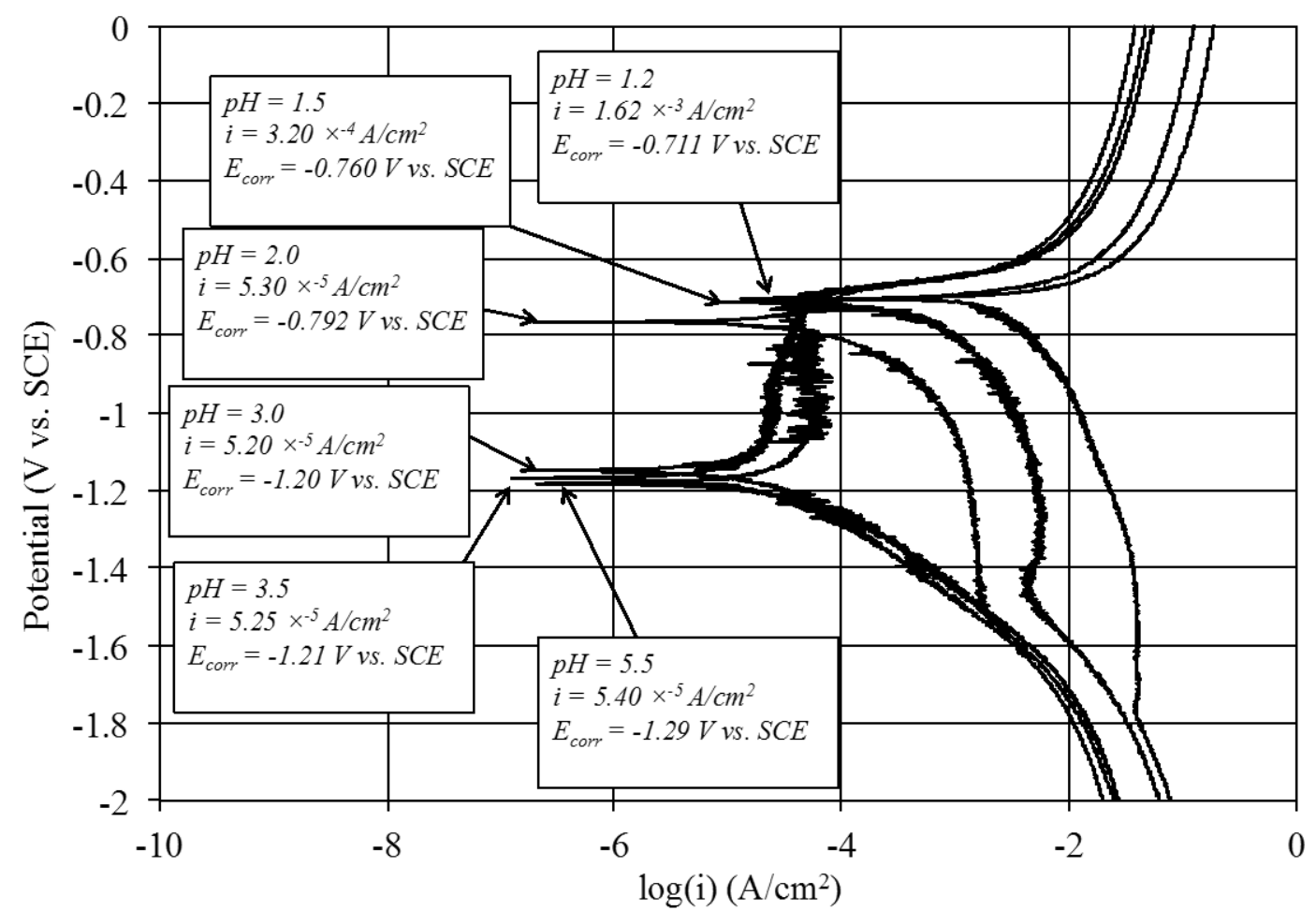

Figure 13: Effect of $\mathrm{pH}$ on the polarization behavior of AA6005A-T6 in aerated 3.5\% $\mathrm{NaCl}$. The values of average corrosion potential and corrosion current density for each solution $\mathrm{pH}$ are indicated.

The corrosion potential of AA6005A-T6 at each value of solution $p H$ was determined by averaging the observed corrosion potentials for at least 5 polarization experiments (Figure 14). The standard deviation in these measurements was taken as the experimental error. A large decrease $(\sim 0.5 V)$ in corrosion potential was observed when the $p H$ of the solution was increased above 2.0. This large shift in corrosion potential was explained by considering the effects of $\mathrm{pH}$ on the stability of the $\mathrm{Al}_{2} \mathrm{O}_{3}$ passive film. Kaesche noted that the high resistivity of an $\mathrm{Al}_{2} \mathrm{O}_{3}$ passive film tended to slow the rate of the cathodic reaction at the surface of the material [24]. Slowing the 
rate of the cathodic reaction would tend to shift the exchange current density of the reaction to a lower value, thereby causing the cathodic polarization curve to intersect the anodic polarization curve at a lower value of potential (similar to Figure 6). When $p H<4.0$, it is known that the passive film on $A l$ becomes thermodynamically unstable (Figure 2), but dissolves very slowly [18]. Therefore, the observed polarization behavior of the material can represent the polarization behavior of the passive films even when $p H<4.0$. The data presented in Figure 14 suggests that under the conditions in these polarization experiments a passive film on AA6005AT6 dissolves slowly when $3.0<p H<5.5$, and is fully dissolved when $p H<2.0$.

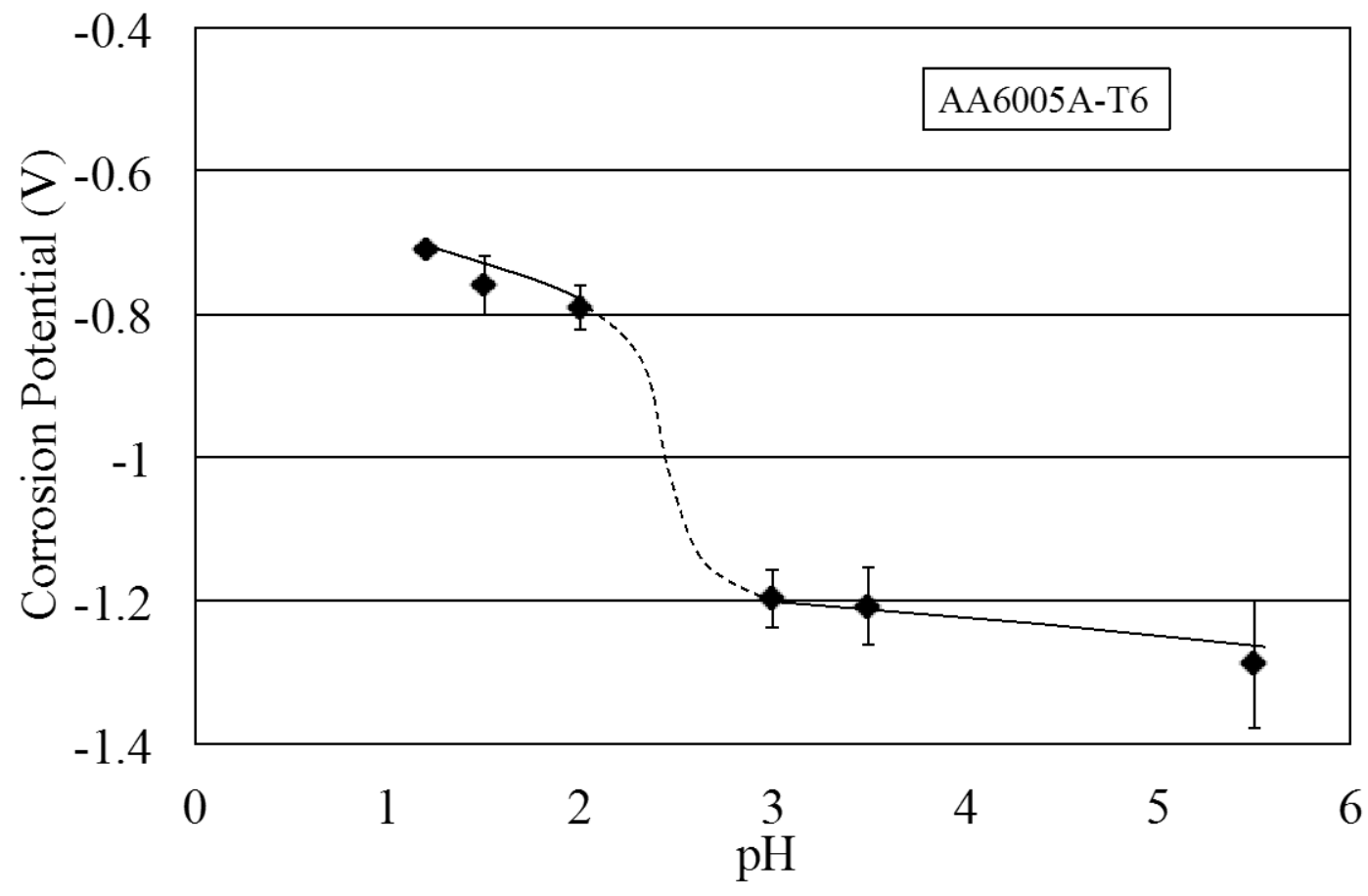

Figure 14:Effect of $\mathrm{pH}$ on the corrosion potential of AA6005A-T6 exposed to an aqueous solution of $3.5 \% \mathrm{NaCl}$. A sharp decrease in corrosion potential was observed when $\mathrm{pH}>2.0$. 
The values of corrosion current density, $i_{\text {Corr }}$, were extracted from polarization curves using the Tafel analysis procedure described in section 2.3 (Figure 4). Tafel analysis was performed on three polarization curves of AA6005A-T6 for each solution $p H$. A representative example of Tafel analysis for a sample of AA6005A-T6 exposed to an aerated saltwater solution at $p H=2.0$ is illustrated in Figure 15. Notice the very small range of linearity in the anodic portion of the polarization curve. The small range of linearity in the anodic portion of the polarization curve resulted in some error in determining values of $i_{\text {Corr }}$. The experimental error in $i_{\text {Corr }}$ was determined by calculating the standard deviation for at least 5 values of measured current density from each of the experimental conditions. 


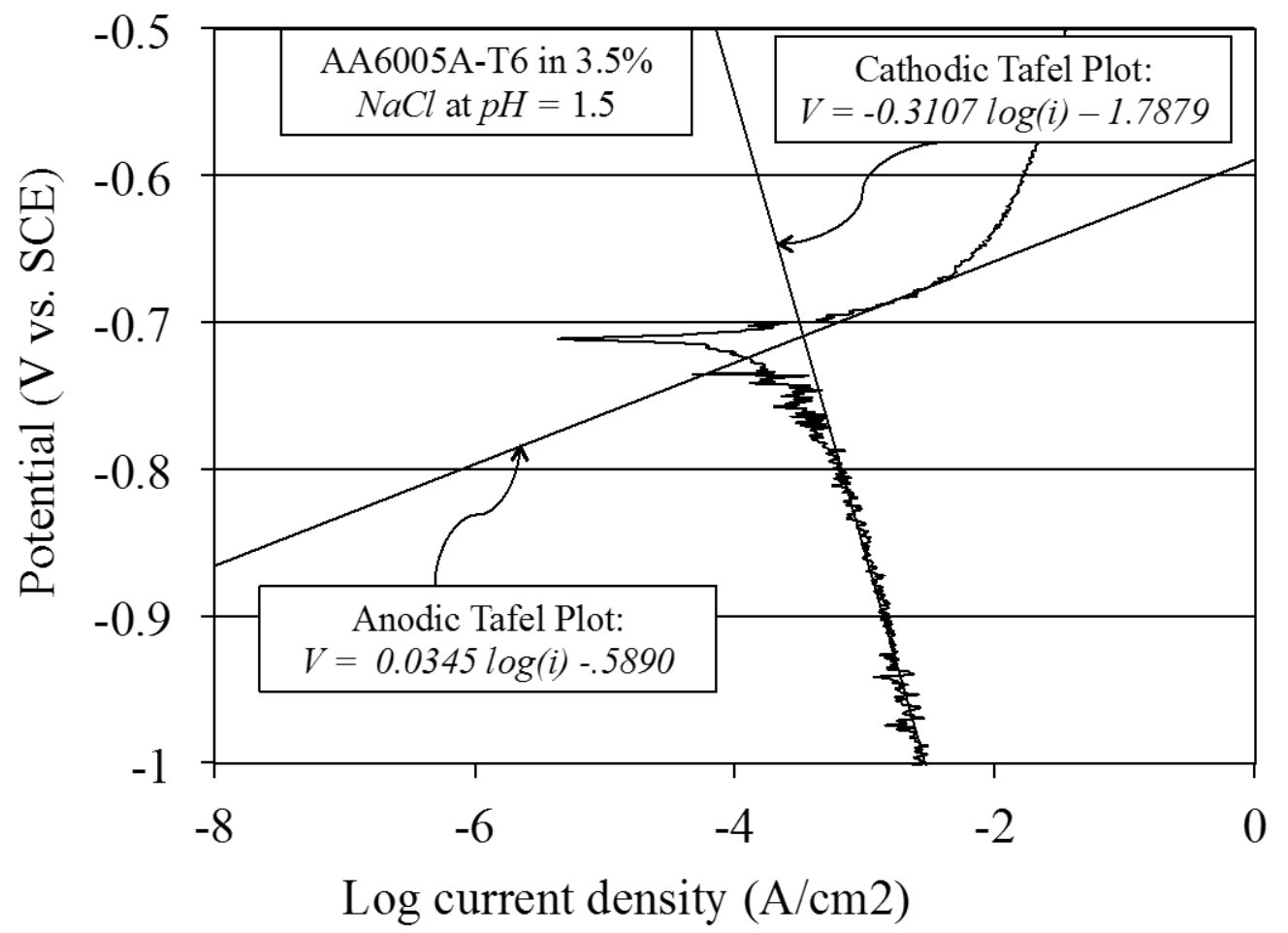

Figure 15: Representative Tafel analysis for AA6005A-T6 exposed to a solution of $3.5 \% \mathrm{NaCl}$ at $\mathrm{pH}=2.0$.

The relationship between solution $p H$ and $i_{\text {Corr }}$ is illustrated in Figure 16. The corrosion current density varied with solution $p H$. Corrosion current densities were found to decrease by approximately 2 orders of magnitude when the $p H$ was increased from 1.2 to 2.0. When the solution $p H$ was greater than 2.0 , the corrosion current remained relatively constant at approximately $5.3 \times 10^{-5} \mathrm{~A} / \mathrm{cm}^{2}$. 


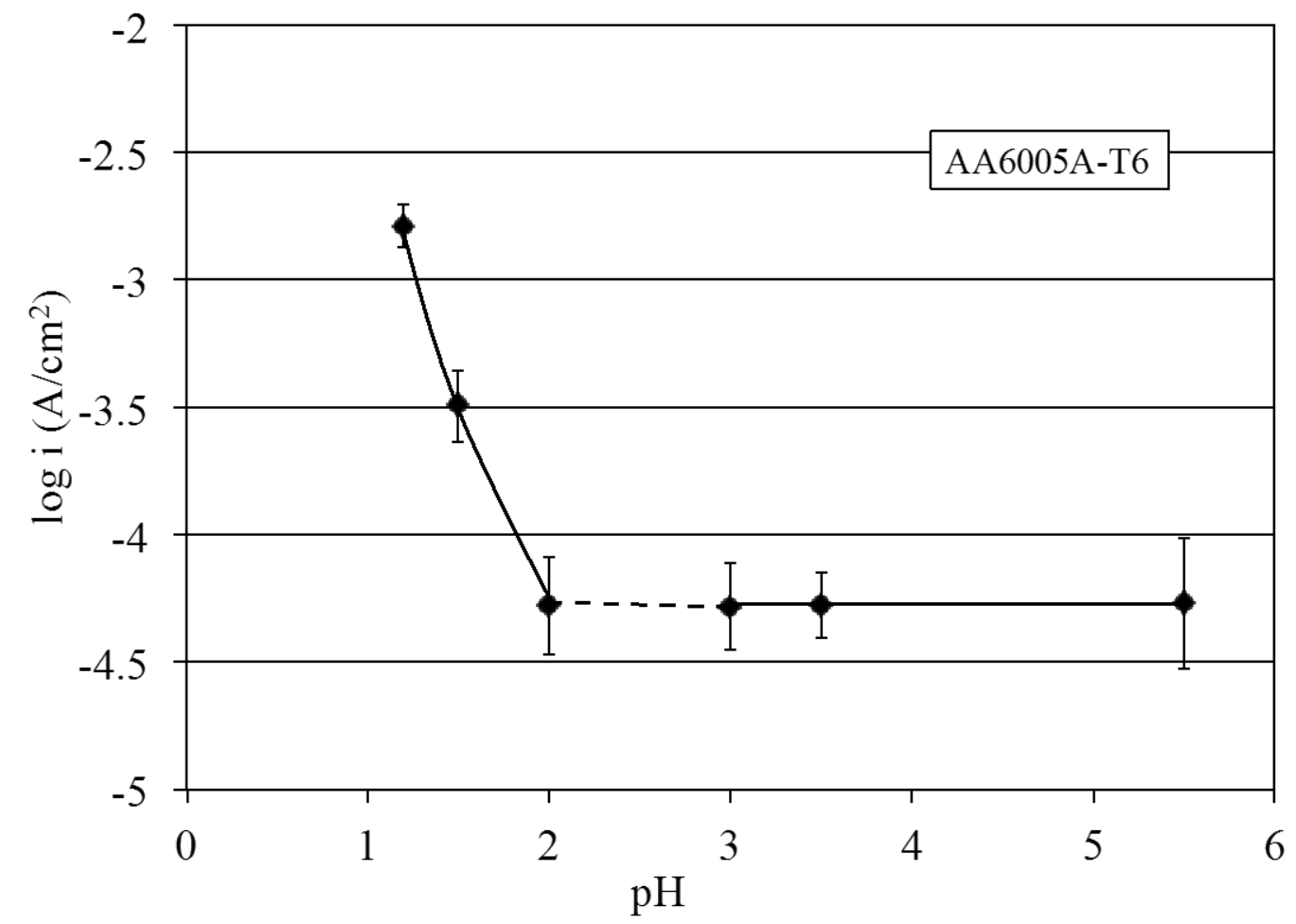

Figure 16: Effect of solution $p H$ on the corrosion current of AA6005A-T6 exposed to an aqueous solution of $3.5 \% \mathrm{NaCl}$. The corrosion current decreased by two orders of magnitude when $1.2 \leq p H \leq 2.0$, and remained near constant at approximately $5.3 \times 10^{-5}$ when $2.0 \leq p H \leq 5.5$.

The effect of heat treatment on the polarization behavior of AA6005A extrusions was examined by performing polarization measurements on samples in a T4, T6, or OA temper in solutions of $3.5 \% \mathrm{NaCl}$ with $1.20<p H<5.50$. Figure 17 illustrates the effects of heat treatment on the polarization behavior of AA6005A exposed to a solution at $p H=1.50$. Heat treatment does not appear have a significant influence on the bulk polarization behavior of the material. A similar relationship was found to be true for solutions with $1.20<p H<5.50$. These results suggest that 
electrochemical reactions participating in the overall corrosion reaction do not depend significantly on the heat treatment of the material.

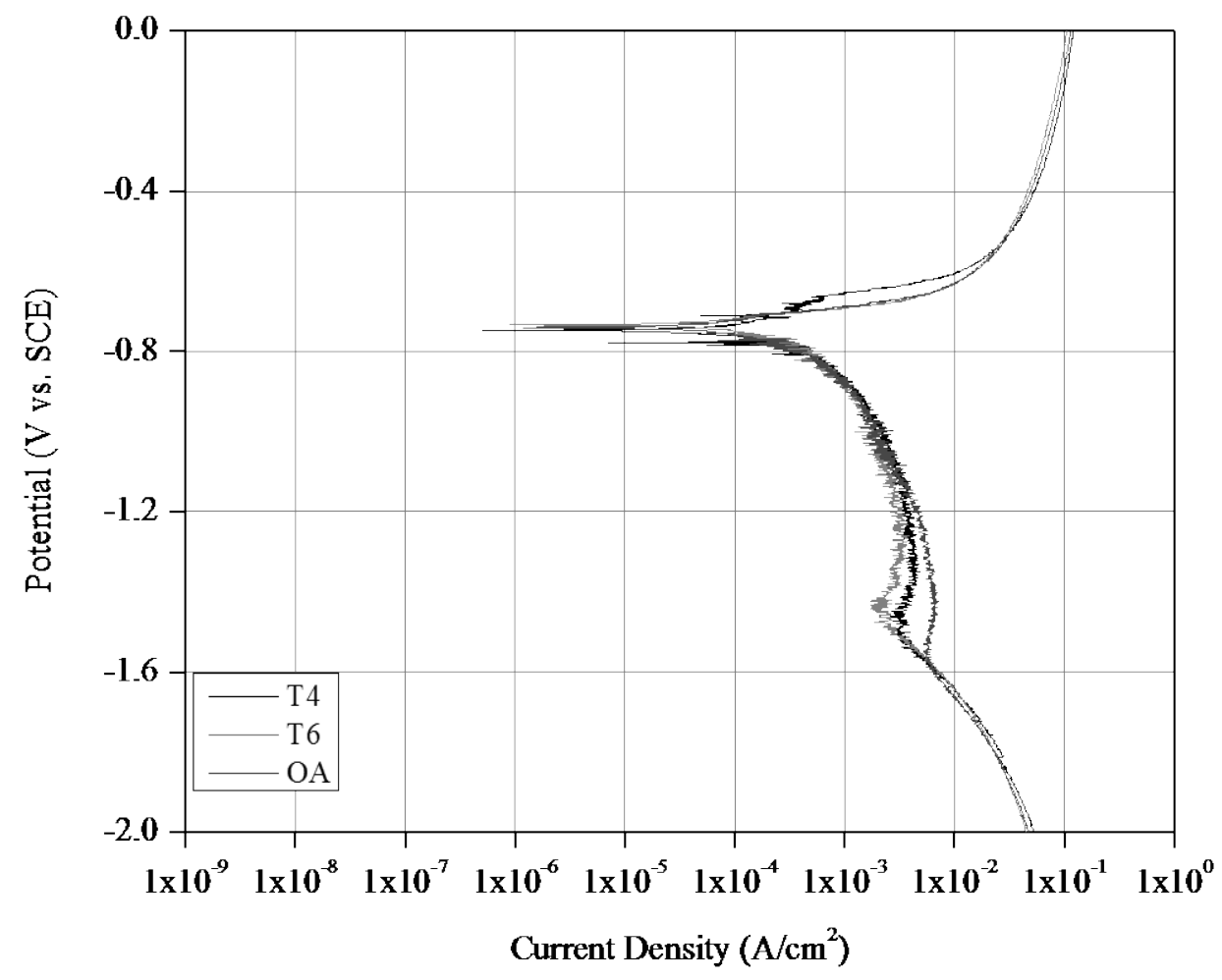

Figure 17: Effect of heat treatment on the polarization behavior of AA6005A extrusions exposed to $3.5 \% \mathrm{NaCl}$ at $\mathrm{pH}=1.50$. The overall polarization does not vary significantly with heat treatment, which indicates that cathodic and anodic reactions involved in the overall corrosion reaction do not depend on heat treatment.

The effect of adding a hydrogen recombination poison to an aerated saltwater solution on the polarization behavior of AA6005A-T6 is illustrated by polarization curves in Figure 18. Adding $A s^{3+}$ to $p H=1.50$ solution resulted in a slight decrease in $E_{C o r r}$ and $i_{C o r r}$ when the bulk solution $p H$ was 1.50 . When the bulk solution $p H$ was 
3.00, the presence of $A s^{3+}$ resulted in a larger decrease in $E_{\text {Corr }}$ and $i_{\text {Corr }}$. Note that there is some noise near $E_{\text {Corr }}$ in the polarization curve for the sample tested without $\mathrm{As}_{2} \mathrm{O}_{3}$ at $p H=3.00$. This noise is random, and does not represent the actual polarization behavior of the material. The presence of a hydrogen recombination poison should not alter the operating anodic reaction; therefore these results suggest that $A s^{3}$ may alter the cathodic reaction slightly. It is known that dissolved $A s^{3+}$ tends to slow the recombination of $H_{a d s}$, thereby slowing the kinetics of the cathodic reaction $[20,21,63]$. The results presented in Figure 18B are consistent with this assertion; the lower observed values of $i_{\mathrm{Corr}}$ and $E_{\mathrm{Corr}}$ with the addition of $\mathrm{As}_{2} \mathrm{O}_{3}$ to the aerated saltwater solutions suggest a decrease in the exchange current density for the cathodic reaction. These results also suggest that adding $\mathrm{As}_{2} \mathrm{O}_{3}$ to the aerated saltwater solutions may be an effective method to test qualitatively for a hydrogen embrittlement mechanism of SCC in AA6005A extrusions. 

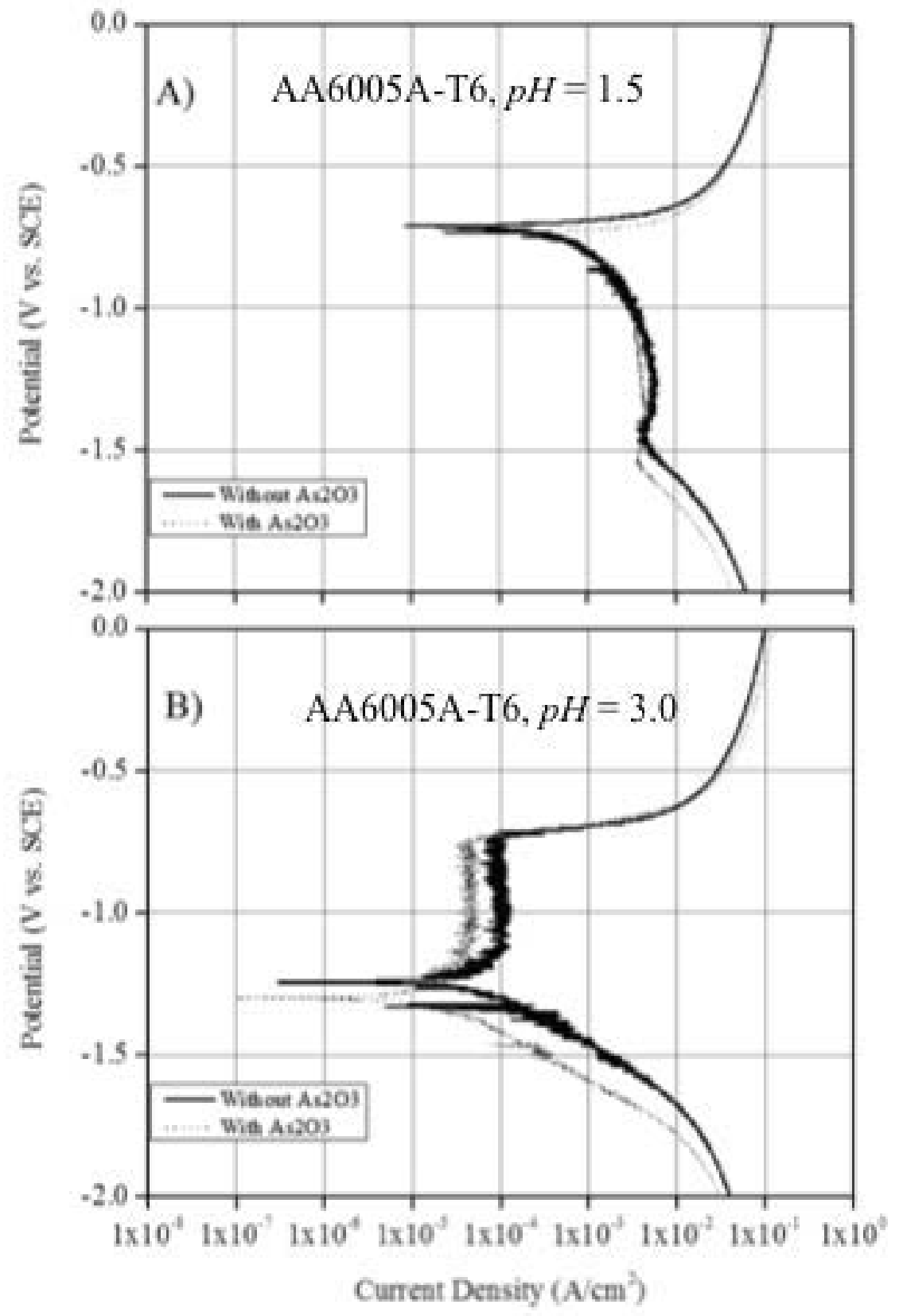

Figure 18: Effect of a hydrogen recombination poison on the polarization behavior of AA6005A-T6. A) The presence of $\mathrm{As}_{2} \mathrm{O}_{3}$ does not alter the overall corrosion reaction when $\mathrm{pH}=1.50$. B) The presence of $\mathrm{As}_{2} \mathrm{O}_{3}$ decreases the corrosion potential and corrosion slightly current when $\mathrm{pH}=3.00$. The scale for current density is shared for $A$ and $B$. 


\subsection{IGC of the Elongated Grain Structure in AA6005A}

\subsubsection{Experimental Methods - IGC of AA6005A}

The effects of heat treatment and environment on IGC susceptibility of the elongated grain structure in AA6005A extrusions were studied by conducting immersion tests. All immersion tests were carried out in solutions of $3.5 \% \mathrm{NaCl}$ with $1.20<p H<2.00$. The $p H$ was adjusted using $H C l$. Samples were tested in a T4, T6, or OA temper.

Rectangular samples measuring $2.54 \mathrm{~cm} \times 1.27 \mathrm{~cm}$ were machined using a $\mathrm{CNC}$ end mill. The extrusion surfaces (1-3 planes) were ground and polished to a $1 \mu \mathrm{m}$ finish to fully remove the equiaxed layer. The machined surfaces (1-2, and 2-3 planes) were polished to a $1 \mu \mathrm{m}$ finish to limit the effects of residual stresses created from the machining process.

All samples were degreased in a solution containing $5 \% \mathrm{NaOH}$ at $50-60^{\circ} \mathrm{C}$ for $5 \mathrm{~min}$. This degreasing procedure served two primary purposes: 1) To remove any residual organic matter on the surface of the material, and 2) To expose IGC initiation sites (e.g. constituent particles). The degreased samples were rinsed in laboratory ethanol and water and stored in a desiccator prior to immersion testing. The samples were stored for sufficiently long periods of time prior to immersion testing to allow repassivation of the alloy. All samples were handled with gloved hands, and care was taken to avoid any contamination on the sample surfaces subsequent to degreasing. Immersion testing was conducted by suspending the samples in the test solution using monofilament fishing line for a times ranging from 2 to $576 \mathrm{hrs}$. The 
modes and depths of corrosion attack along all three directions (Figure 10) were characterized using standard metallographic practices.

The structure and morphology of the grain boundary precipitates in the elongated grain structure was characterized using transmission electron microscopy (TEM) and energy dispersive $\mathrm{x}$-ray spectroscopy (EDS). The purpose of the TEM examination was to determine if there is a relationship between grain boundary microstructure and IGC susceptibility. TEM foils were prepared by thinning samples of all age treatments to approximately $100 \mu \mathrm{m}$ by abrasive grinding and polishing. Small (3 $\mathrm{mm}$ diameter) disks were punched from the thinned samples. Final thinning was conducted using a double-jet electropolisher. The electrolyte was a solution consisting of $25 \% \mathrm{HNO}_{3}$ and $75 \%$ methanol. The temperature of the electrolyte was held at $-25{ }^{\circ} \mathrm{C}$ during electropolishing. Electropolishing was carried out $30 \mathrm{~V}$.

\subsubsection{Results and Discussion - IGC of AA6005A}

The mode of corrosion attack in the elongated grain structure when tested in a solution of $3.5 \% \mathrm{NaCl}$ at $\mathrm{pH}=1.20$ was found to depend on heat treatment, as illustrated in Figure 19. When the alloy was in a T4 temper, uniform IGC was observed along directions in the elongated grain structure. When the alloy was in a T6 temper, a IGC was observed along all directions, though there was some evidence of pitting. When the alloy was in an OA temper, local pitting was observed along all directions. The relationship between post-extrusion thermal treatment and mode of corrosion attack in the elongated grain structure observed here is similar to that reported in the equiaxed grain structure $[4,7]$. 

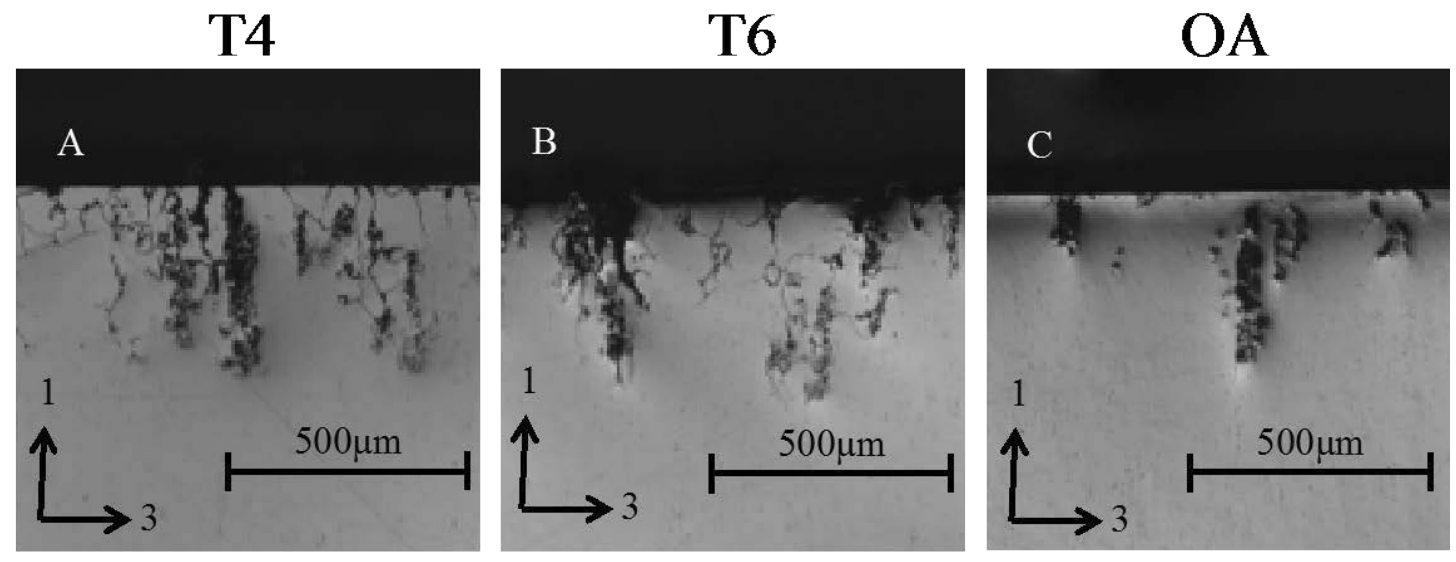

Figure 19: Effect of age treatment on the mode of corrosion attack along the extrusion direction. All samples were tested in $3.5 \% \mathrm{NaCl}$ at $\mathrm{pH}=1.20$ for 24 hrs. A) Pure IGC was observed when the material was in a T4 temper. B) IGC with some evidence of pitting was observed when the material was in a T6 temper. C) Local pitting was observed when the material was in an OA temper.

Transmission electron microscopy revealed that the morphology of the grain boundary precipitates in the elongated grain structure depends on heat treatment. A continuous film containing an excess of $C u$ was observed in samples in a T4 condition (Figure 20). The $\mathrm{Cu}$-rich film was still present when the material was in a T6 condition, but there was evidence that the film began to coarsen to form grain boundary precipitates adjacent to the film (Figure 21). EDS analysis demonstrated that the grain boundary precipitates contained an excess of $\mathrm{Cu}, \mathrm{Mg}$, and $\mathrm{Si}$. The $\mathrm{Cu}$ rich film had completely transformed into discrete grain boundary precipitates containing large amounts of $\mathrm{Cu}, \mathrm{Mg}$, and $\mathrm{Si}$ when the material was in an OA condition (Figure 22). 


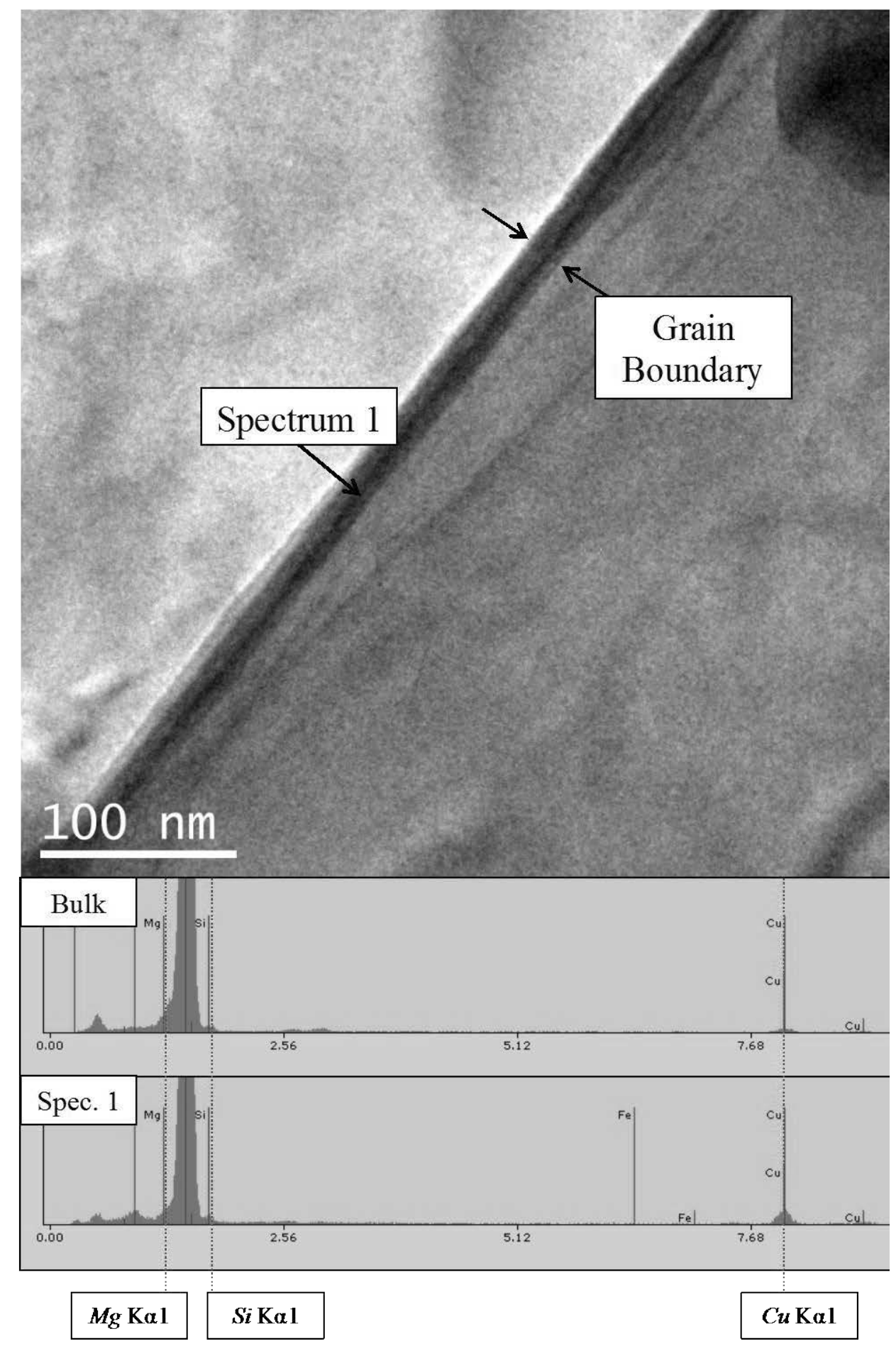

Figure 20: Core grain boundary microstructure of AA6005A-T4. A continuous film with elevated $\mathrm{Cu}$ content was observed along the grain boundaries. 


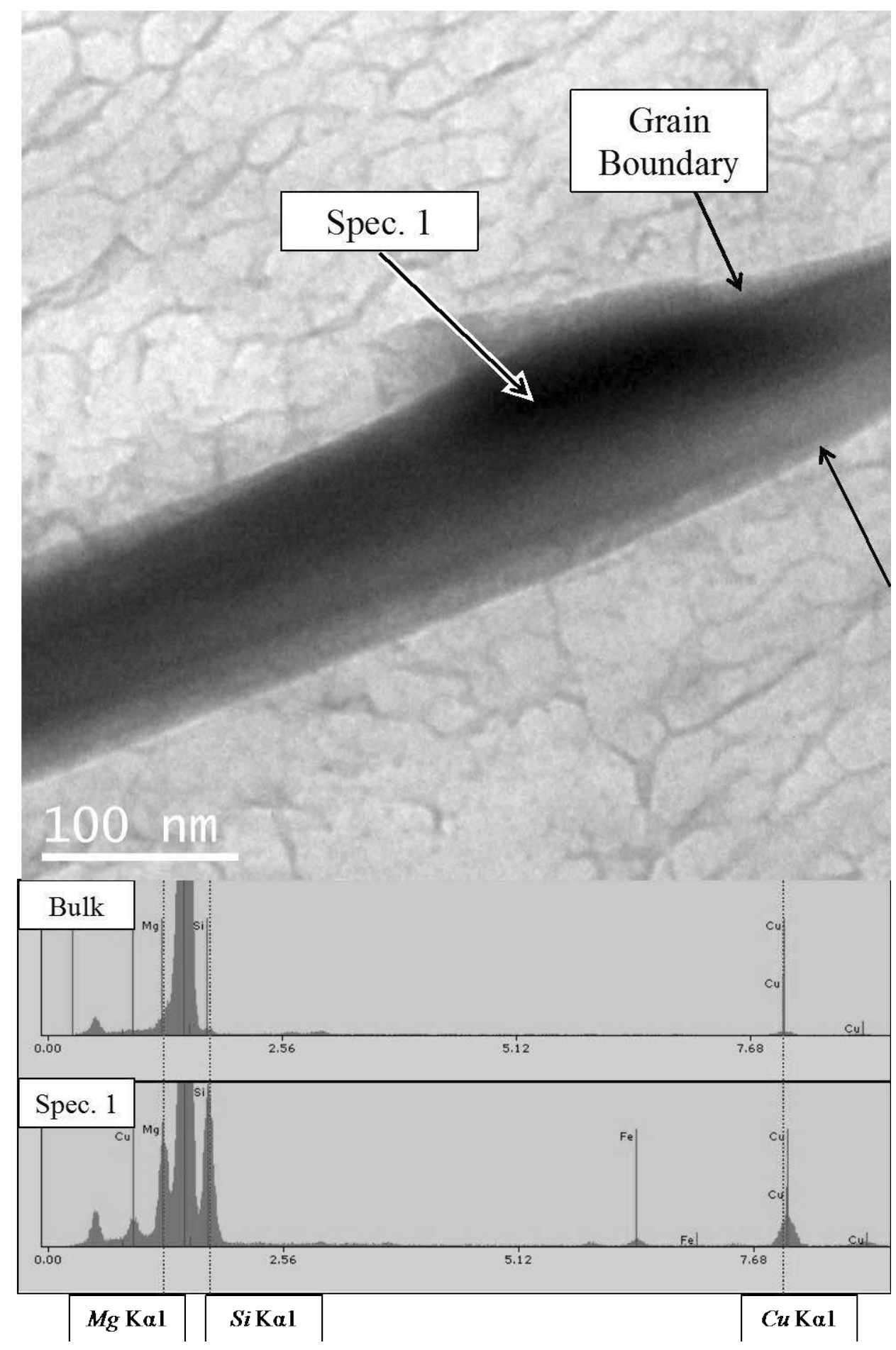

Figure 21: Core grain boundary microstructure of AA6005A-T6. Elevated Cu, Mg, and Si contents were observed along the grain boundary. The Cu-rich film was present along the grain boundaries, but was beginning to coarsen to form discrete particles upon aging to a T6 condition. 


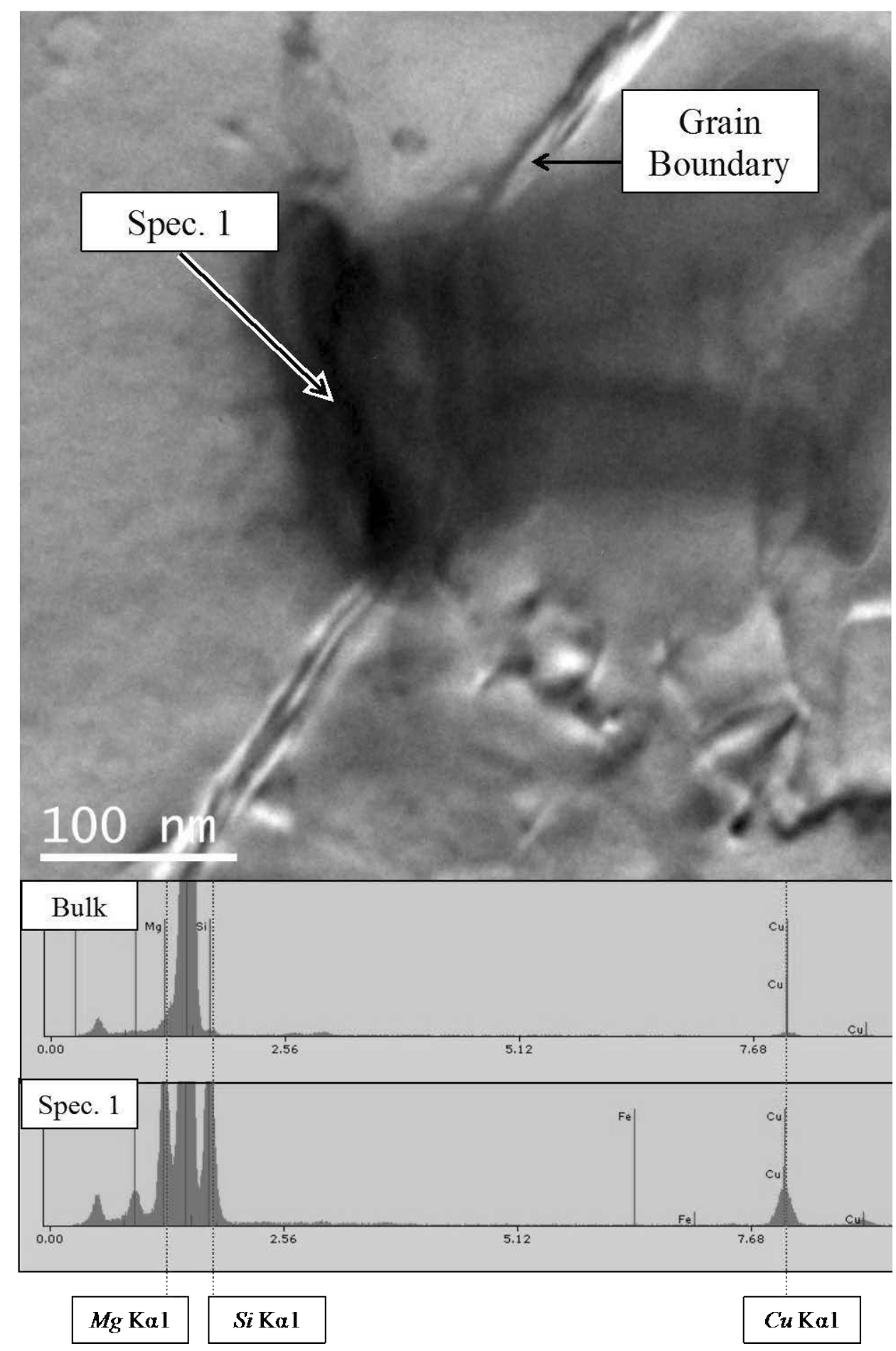

Figure 22: Core grain boundary microstructure of AA6005A-OA. There was no evidence of a continuous film along the grain boundary. The Cu-rich film had coarsened with the overaging heat treatment to form discrete grain boundary precipitates with an excess of $\mathrm{Mg}$ and $\mathrm{Si}$. 
Recall that Nisanciaglu et al. attributed IGC susceptibility in the equiaxed grain structure of AA6005A to the presence of a continuous $\mathrm{Cu}$-rich film along the grain boundaries [4-7].These researchers determined that the $\mathrm{Cu}$-rich film was a $Q-$ phase $\left(\mathrm{Al}_{4} \mathrm{Mg}{ }_{8} \mathrm{Si} 7 \mathrm{Cu}\right)$ precipitate, which was noble relative to the $\mathrm{PFZ}$, thereby resulting in IGC susceptibility by microgalvanic coupling between the grain boundary precipitates and the PFZ. Results presented in Figures 19-22 are consistent with Nisanciaglu's argument. A continuous $\mathrm{Cu}$-rich film was observed along the grain boundaries in the elongated structure when the material was in a T4 and T6 temper, and the elongated structure was susceptible to IGC in these conditions. When the material was in an OA temper, the $\mathrm{Cu}$-rich film was fully transformed into discrete grain boundary precipitates and the primary mode of corrosion attack was pitting.

The effects of $p H$ on the severity of corrosion attack along the extrusion direction are illustrated in Figure 23. Increasing the $p H$ of the solution to 1.50 yielded similar depth and severity of corrosion attack in the elongated grain structure as was observed in when the material was tested in a $p H=1.20$ saltwater solution. Negligible corrosion attack was observed when the samples were exposed to a saltwater solution at $p H=2.00$. The large decrease in severity IGC attack with increasing $p H$ is consistent with the polarization results that indicated a decrease in corrosion when the solution $p H$ was increased to 2.00 from 1.50 (Figure 16). 

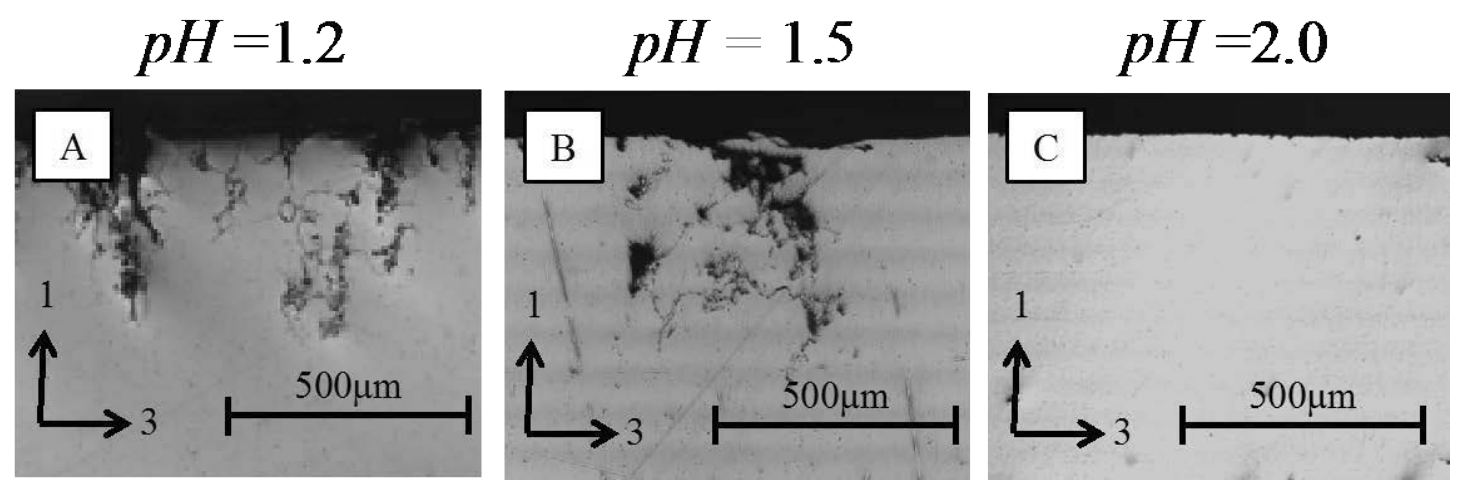

Figure 23: Effect of $\mathrm{pH}$ on the severity of IGC in AA6005A-T6 exposed to 3.5\% $\mathrm{NaCl}$ at A) $p H=1.2, B) p H=1.5$, and $C$ ) $p H=2.0$

Corrosion testing revealed that the depth of corrosion attack is highly anisotropic in the elongated grain structure when the material was in a T4 or T6 temper (Figure 24). Intergranular attack tended to penetrate most rapidly along direction $\mathbf{1}$, and least rapidly along direction $\mathbf{2}$. This anisotropic behavior is graphically illustrated in Figure 25 and Figure 26. Two possible origins for this anisotropic corrosion attack were considered. The first is that the thermomechanical processing (extrusion + heat treatment) might result in strong texturing in the elongated grain structure, thereby yielding in longitudinal grain boundary structures that are more susceptible to IGC due than the grain boundary structures along directions $\mathbf{2}$ and $\mathbf{3}$. The second possible reason investigated is that the corrosion anisotropy simply results from elongation of the grains of the extrusion core. The elongated grains provide a less tortuous intergranular path along the extrusion direction than in the transverse or through-thickness directions. Experiments designed to test these two concepts are detailed in the following paragraphs. 

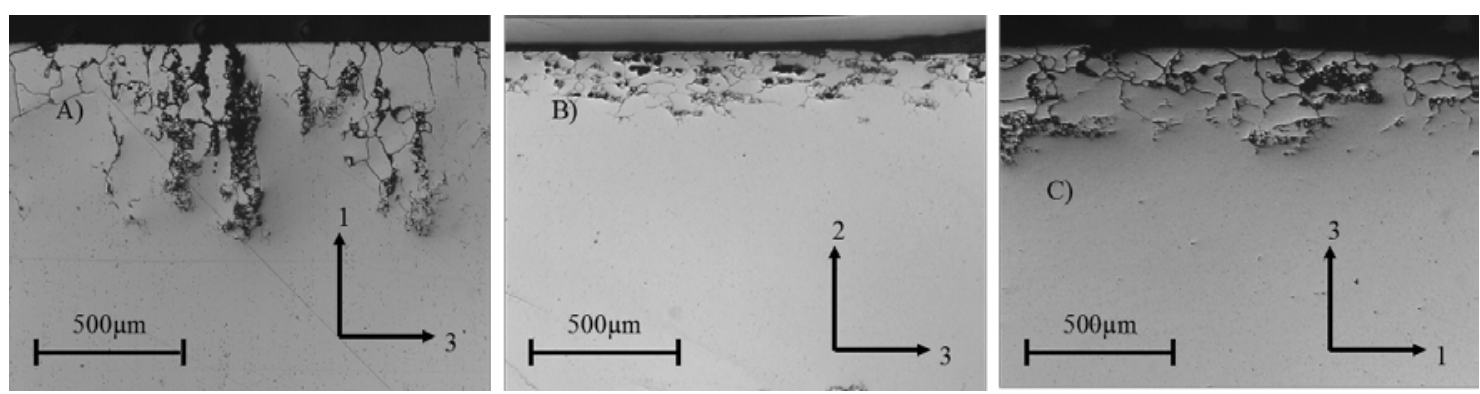

Figure 24: Typical anisotropic IGC attack observed in the elongated grain structure of AA6005A-T4. A) IGC along direction 1. B) IGC along direction 2. C) IGC along direction 3.

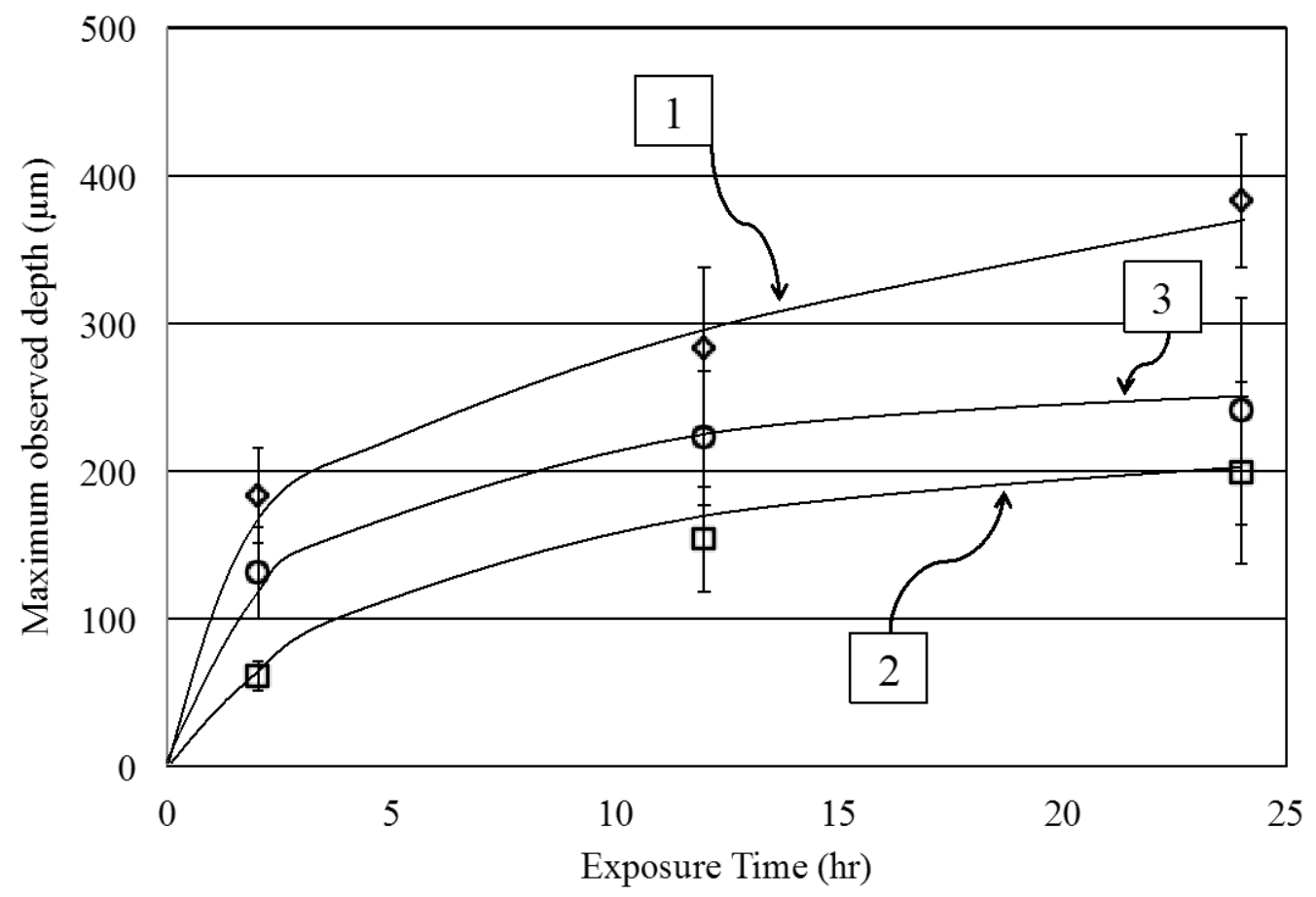

Figure 25: Maximum observed depth of IGC attack in the elongated grain structure along directions $1(\diamond), 2(\square)$, and 3(O) for AA6005A-T4. 


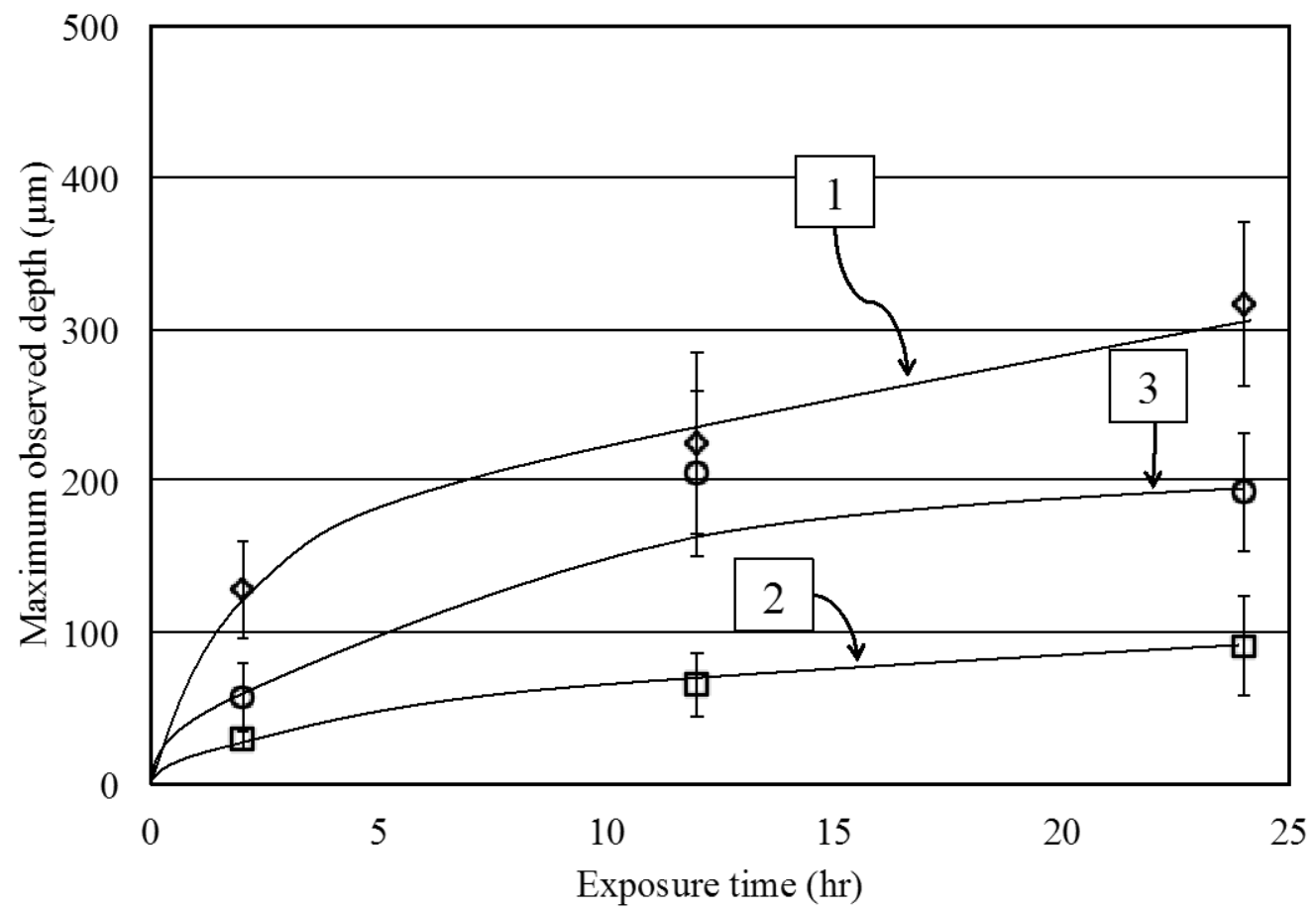

Figure 26: Maximum observed depth of IGC attack in the elongated grain structure along directions $1(\diamond), 2(\square)$, and $3(\boldsymbol{O})$ for AA6005A-T6.

The texture of the elongated grain structure was determined by generating (111), (200), and (220) X-Ray pole figures on a surface normal to direction 2 using $\mathrm{Cu} \mathrm{K} \alpha$ radiation. Texture analysis was conducted on samples having both T4 and T6 heat treatments. Electron backscatter diffraction (EBSD) was also conducted on surfaces normal to direction 2 in specimens with both T4 and T6 age treatments. The EBSD analysis was conducted to supplement the X-Ray pole figure results, and to better understand the effects of grain boundary misorientation on the corrosion susceptibility of the specimens.

The (200) X-Ray pole figure of the elongated grain structure in AA6005A-T6 is illustrated in Figure 27. Clustering of X-Ray intensity near the center of the pole 
figure and at angles of $\varphi=90^{\circ}, 180^{\circ}, 270^{\circ}$ and $360^{\circ}$ to the extrusion direction demonstrate that elongated grain structure has strong cubic texturing with the [100] direction being aligned with the extrusion direction. The texture of the elongated grain structure in AA6005A-T4 was found to be nearly identical to that of AA6005AT6. Similar texture results were found by EBSD analysis. Such strong cubic texturing is typical in extruded $A l$ alloys [64]. These results indicate that directions $\mathbf{1}, \mathbf{2}$, and $\mathbf{3}$ are crystallographically equivalent. If grain boundary structure as influenced by texture was the primary factor influencing the anisotropic corrosion attack, the corrosion attack should be similar along all three directions. Grain boundary structure as influenced by crystallographic texturing does not therefore appear to be the primary source of the observed anisotropy of corrosion attack in the elongated grain structure. 


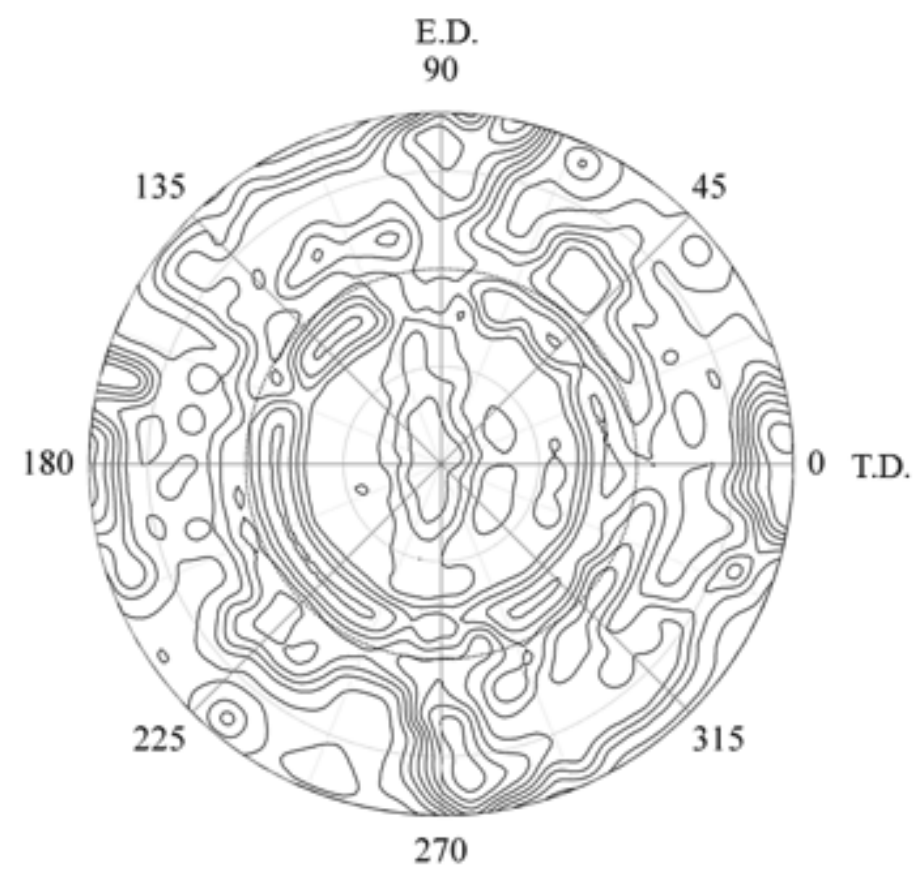

Figure 27: (200) X-Ray pole figure of elongated grain structure in AA6005A. "E.D." indicates the extrusion direction, and "T.D." indicates the transverse direction. Clustering of intensities at $\phi=0^{\circ}, 90^{\circ}, 180^{\circ}$, and $270^{\circ}$ indicates that the elongated structure has strong cubic texturing such that directions 1,2 , and 3 are crystallographically equivalent.

The simple three-dimensional model shown in Figure 28, similar to that employed by Zhang [15], was developed to explain the observed anisotropic corrosion attack in the elongated grain structure of AA6005A extrusions. In this model, grains are represented as three-dimensional rectangular "bricks", with edge lengths corresponding to nominal grain dimension in each direction, stacked upon one another. The nominal grain dimensions for the elongated grain structure in AA6005A-T4 and AA6005A-T6, as measured using a point intersect technique, were $186+/-88 \mu m$ along direction $\mathbf{1}, 47+/-5 \mu m$ along direction $\mathbf{2}$, and 117+/-50 $\mu \mathrm{m}$ along direction 3. The direction of corrosion penetration in Figure 28 is always along the $x$ - 
direction, and the values of $d_{x}, d_{y}$ and $d_{z}$ will change to represent penetration along direction's 1,2 and 3. This is necessary because in the simple grain arrangement of Figure 28, only the $x$-direction approximates the tortuosity associated with corrosion penetration in the real microstructure. Equation 6 relates the length of the corrosion path along direction $x\left(L_{x}\right)$ to the nominal dimensions of the grains.

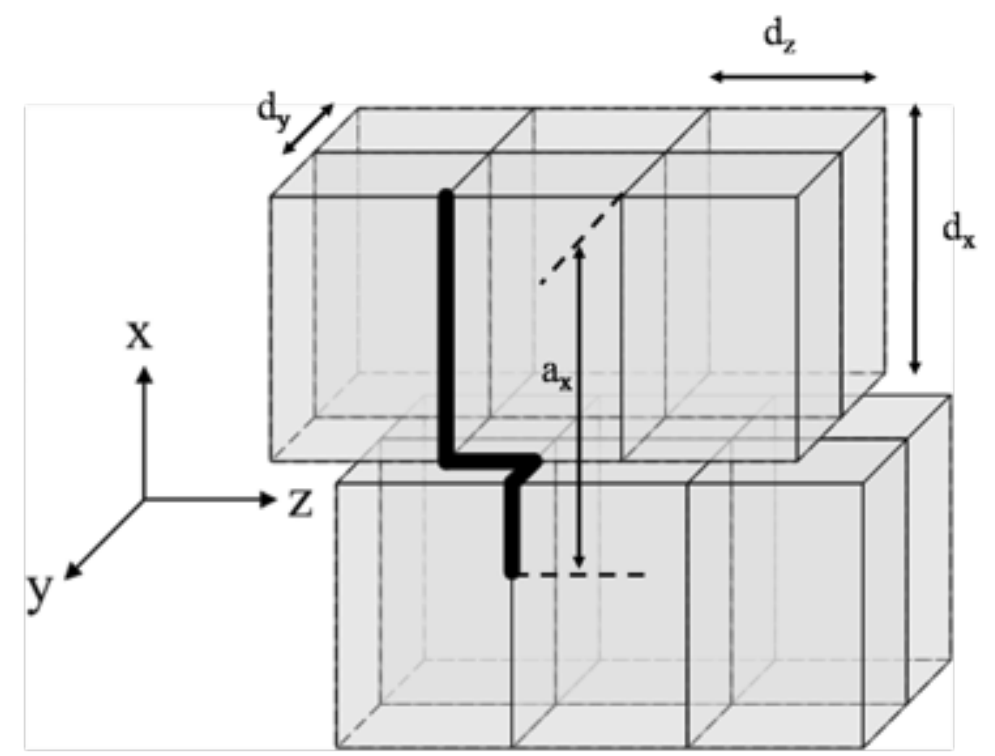

Figure 28: Three-dimensional geometric model used to describe the anisotropic corrosion attack in the elongated grain structure of AA6005A extrusions. The nominal grain dimensions along directions $\boldsymbol{x}, \boldsymbol{y}$, and $\boldsymbol{z}$ are $d_{x}, d_{y}$, and $d_{z}$, respectively. The maximum observed depth of attack along direction $\boldsymbol{x}$ is $a_{x}$. The shortest corrosion path to achieve a depth of attack equal to $a_{x}$ is indicated by the bold black line. The heavy black line represents the corrosion path length $\left(L_{x}\right)$, as measured by this model, and is given in Equation 6.

Equation 6

$$
L_{x}=a_{x} \times\left(1+\frac{d_{y}+d_{z}}{2 d_{x}}\right)
$$


The model was tested by comparing the corrosion path lengths $\left(L_{\mathrm{x}}\right)$ deduced from Equation 6 using the measured grain dimensions along each of the three directions and the measured depth of attack, $a_{x}$. If geometric anisotropy of the elongated structure is the only important factor determining the maximum depth of attack, the calculated corrosion path lengths, $L_{x}$, along the three orthogonal directions $(1,2$, and 3$)$ should be similar. Table 6 gives the results from testing the geometric model for AA6005A in the T4 and T6 conditions.

Table 6: Calculated corrosion path lengths along all directions for samples in a T4 and T6 temper

\begin{tabular}{|c|c|c|c|c|c|c|}
\hline \multirow{2}{*}{} & \multicolumn{3}{|c|}{$\mathrm{T} 4$} & \multicolumn{3}{c|}{$\mathrm{T} 6$} \\
\cline { 2 - 7 } $\mathrm{L}_{\mathrm{x}}(\mathrm{mm})$ & \multicolumn{3}{c|}{$\mathrm{L}_{\mathrm{x}}(\mathrm{mm})$} \\
\hline $\begin{array}{c}\text { Exposure Time } \\
\text { (hr) }\end{array}$ & 1 & 2 & 3 & 1 & 2 & 3 \\
\hline \multirow{3}{*}{2} & 0.264 & 0.258 & 0.262 & 0.184 & 0.124 & 0.114 \\
& $+/-$ & $+/-$ & $+/-$ & $+/-$ & $+/-$ & $+/-$ \\
& 0.046 & 0.043 & 0.062 & 0.046 & 0.032 & 0.045 \\
\hline \multirow{3}{*}{24} & 0.408 & 0.648 & 0.444 & 0.324 & 0.276 & 0.408 \\
& $+/-$ & $+/-$ & $+/-$ & $+/-$ & $+/-$ & $+/-$ \\
& 0.078 & 0.149 & 0.091 & 0.086 & 0.090 & 0.109 \\
\hline & 0.552 & 0.840 & 0.480 & 0.456 & 0.384 & 0.408 \\
& $+/-$ & $+/-$ & $+/-$ & $+/-$ & $+/-$ & $+/-$ \\
& 0.064 & 0.259 & 0.153 & 0.078 & 0.138 & 0.077 \\
\hline
\end{tabular}

The calculated corrosion path lengths for T4 samples agree reasonably well directions $\mathbf{1}$ and $\mathbf{3}$ for each of the exposure times. The calculated corrosion path lengths for T4 samples along direction $\mathbf{2}$ are larger than along directions $\mathbf{1}$ and $\mathbf{3}$ for longer exposure times, but still lie within the experimental margin of error. The corrosion path lengths for samples tested in a T6 condition are shorter than those of 
samples tested in a T4 condition, indicating that the rate of IGC is lower with this heat treatment. The calculated corrosion path lengths for T6 samples are in good agreement along all directions and exposure times. These results suggest that the observed anisotropy of corrosion attack in the elongated grain structure of extruded AA6005A is related to the geometric anisotropy of the elongated grains, and that the maximum depth of corrosion attack is ultimately determined by the tortuosity of the IGC path.

Given that the model described above seems to accurately predict the relationship between corrosion path lengths and observed depth of attack, this simple model will be used in subsequent sections to understand the relationship between IGC and SCC susceptibility of AA6005A extrusions. The calculated corrosion path length $\left(L_{x}\right)$ will hereafter be referred to as the "structure-corrected corrosion path length".

\subsubsection{Effects of Diffusion on IGC Velocity}

Additional IGC experiments were conducted with exposure times up to 576 hrs to investigate further the observed decay in IGC velocity with exposure time (Figures 25-26). All of these experiments were conducted on samples of AA6005AT6 exposed to an aerated solution of $3.5 \% \mathrm{NaCl}$ at $\mathrm{pH}=1.50$. The maximum depth of corrosion attack was measured along direction 1 using optical microscopy. Multiple micrographs (at least 5 per sample) were evaluated from three samples to determine the maximum depth of attack for each exposure time.

Figure 29 illustrates the decay in IGC penetration rate with exposure time for AA6005A-T6 exposed to $3.5 \% \mathrm{NaCl}$ at $\mathrm{pH}=1.5$. The instantaneous IGC velocity at a 
given exposure time was determined by taking the derivative of the logarithmic function that was fit to the depth vs. exposure time data. A plot of the structure corrected corrosion velocity $\left(V_{I G C}\right)$ vs. structure corrected corrosion path length $(L)$ is shown in Figure 30. 


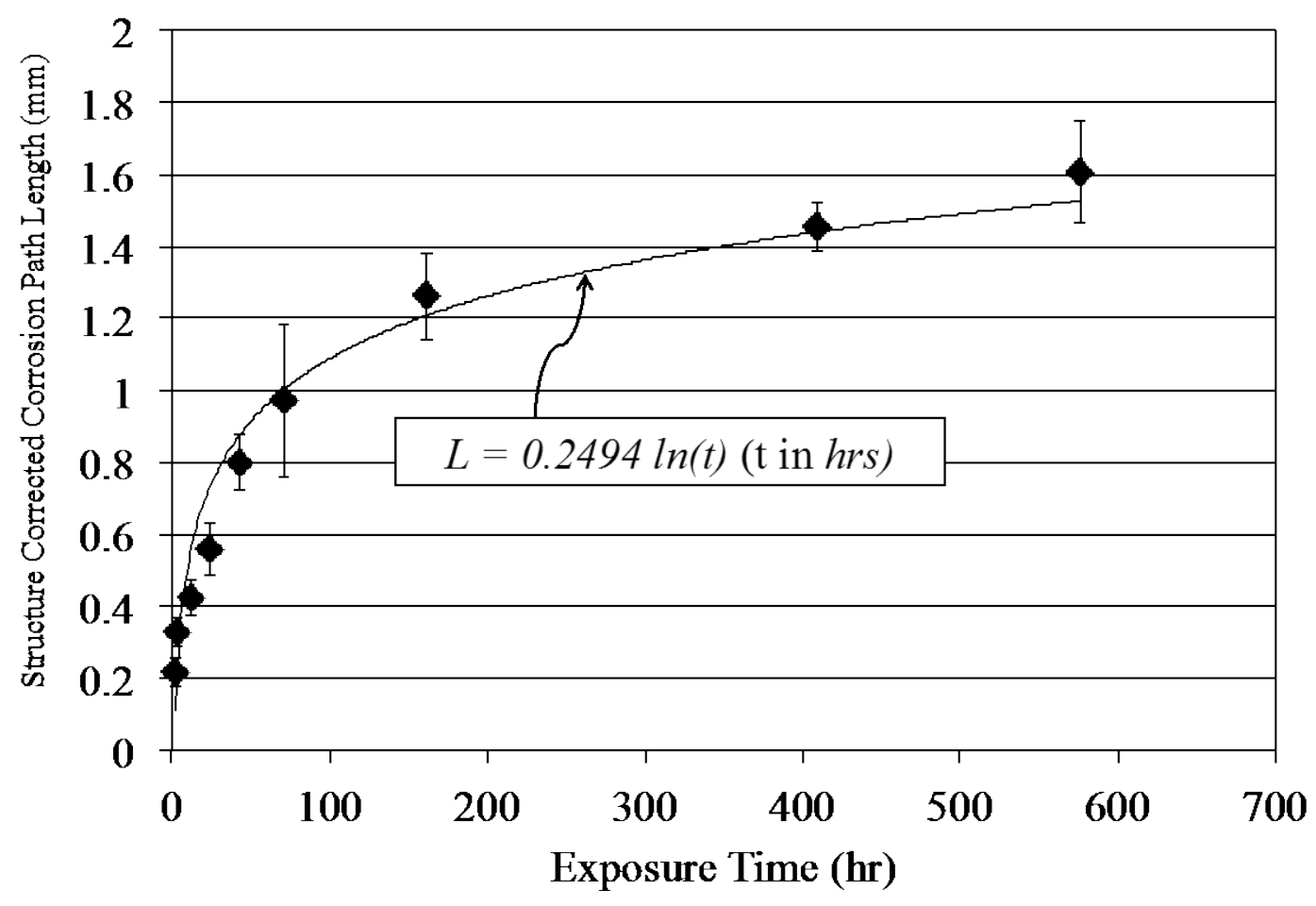

Figure 29: Structure corrected corrosion path length for AA6005A-T6 exposed to an aerated solution of $3.5 \% \mathrm{NaCl}$ at $\mathrm{pH}=1.5$. The rate of IGC penetration decays with exposure time. 


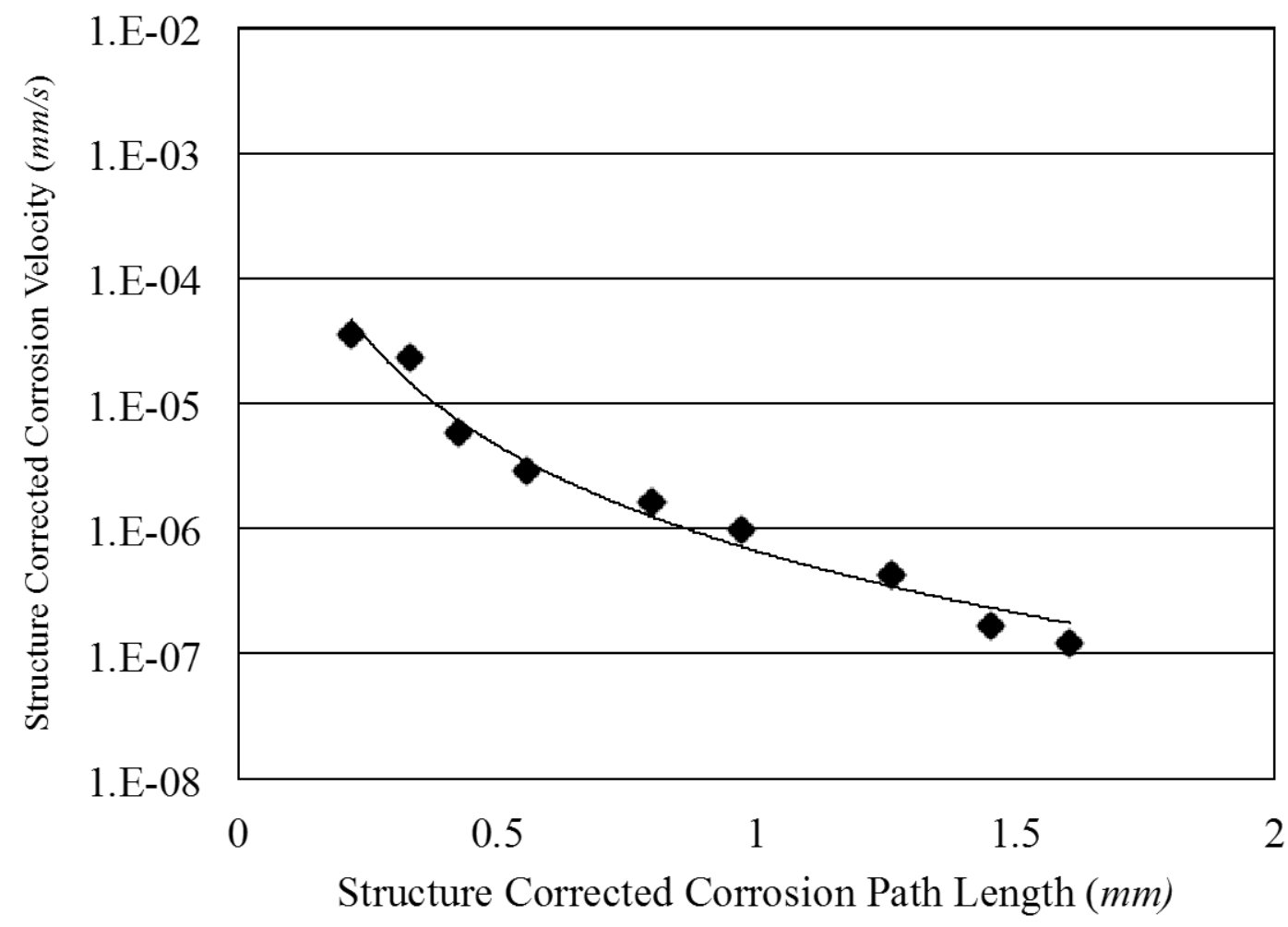

Figure 30: Structure corrected IGC velocity of with increasing corrosion path length observed in AA6005A-T6 exposed to $3.5 \% \mathrm{NaCl}$ at $\mathrm{pH}=1.5$. The velocity of IGC decreases by more than $100 X$ with increasing corrosion path length.

If we assume that the anodic (Equation 1) and cathodic (Equation 2) reactions involved in the overall corrosion reaction do not vary with corrosion path length, the mechanism of dissolution should not change with increasing corrosion path length. The large decrease in IGC velocity observed at larger corrosion path lengths must therefore be due to some factor that limits the kinetics of dissolution at the tip of an IGC path. The effects of diffusion of specific ionic species involved in the corrosion reaction in the electrolyte were considered in developing a model to explain the observed decay in IGC velocity. 
It has been established that the local environment within an IGC (or SCC) path may be different than that of the bulk environment $[39,40,44,62,65]$. Because the different local environment within a corrosion path may significantly influence the local corrosion behavior of the alloy, experiments were performed to characterize the local $p H$ and $\left[\mathrm{Al}^{3+}\right]$ within an occluded region of AA6005A-T6, and examine how these factors may affect the velocity of IGC attack.

The effect of corrosion path length on the local $p H$ at the crack tip was qualitatively examined using the sample configuration illustrated in Figure 31. A shallow $5.5 \mathrm{~mm}$ wide groove was machined into the surface of the sample to accept a piece of litmus paper. A small triangular $0.1 \mathrm{~mm}$ deep channel was machined into the bottom of the groove. The channel was designed to simulate the dimensions of an IGC path. Litmus paper was placed into the groove, and glass slides were attached with spring clips to seal the top and bottom surfaces of the sample. The spring clips did not contact the sample; therefore any possible galvanic effects were avoided. The prepared sample was placed in an aerated solution of $3.5 \% \mathrm{NaCl}$ at $p H=1.50$. The solution level was maintained at the top of the $0.1 \mathrm{~mm}$ deep channel. The change in $p H$ vs. distance from the free surface was approximately determined by observing the change in color of the litmus paper over time. Typical results of this analysis are summarized in Figure 32. 

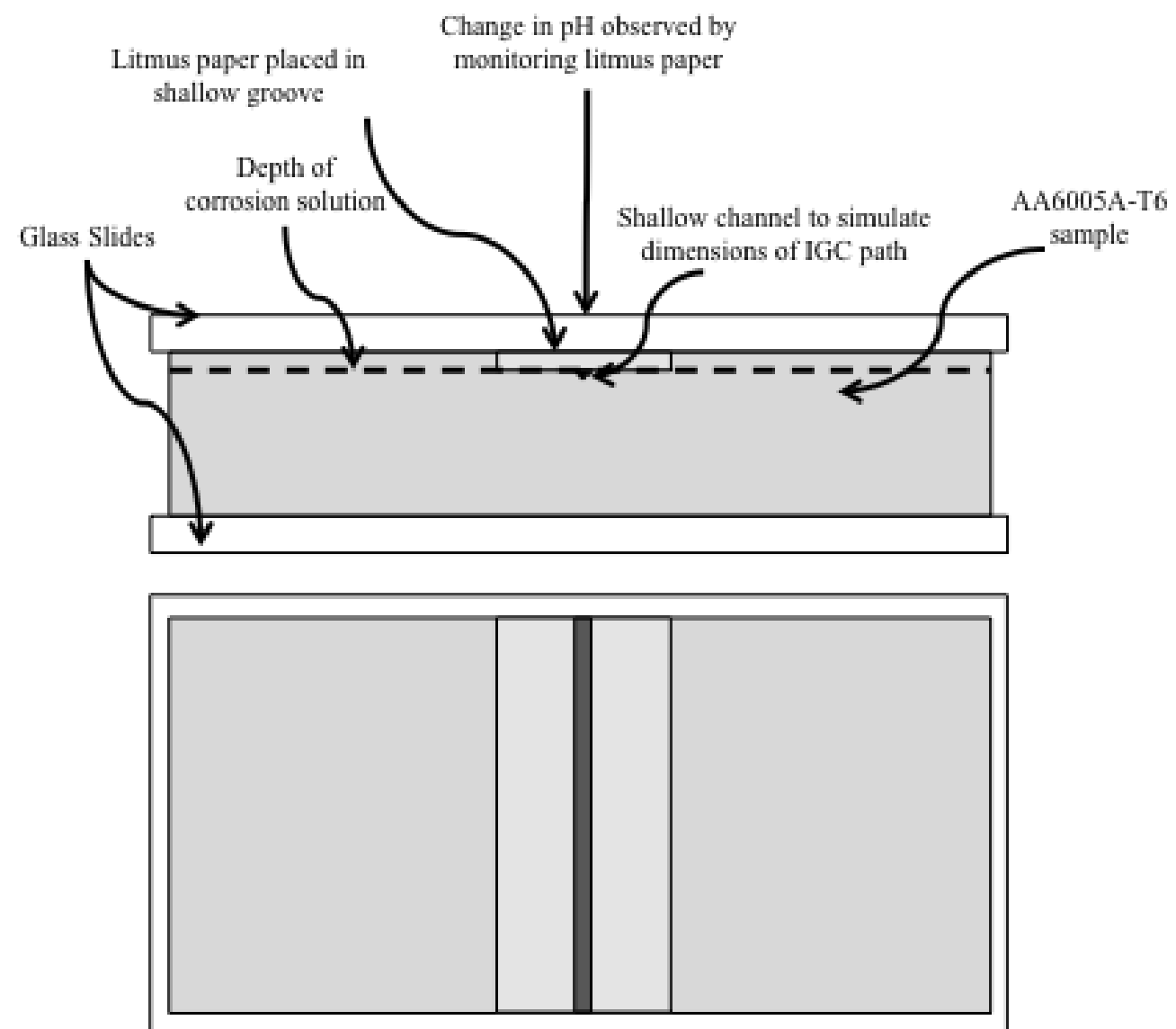

Figure 31: Sample configuration used to qualitatively determine the variation in $\mathrm{pH}$ with corrosion depth. A piece of litmus paper was placed in a shallow groove, and the sample was exposed to the test environment. The change in color of the litmus paper was used to qualitatively determine the $\mathrm{pH}$ gradient along a simulated IGC path. 

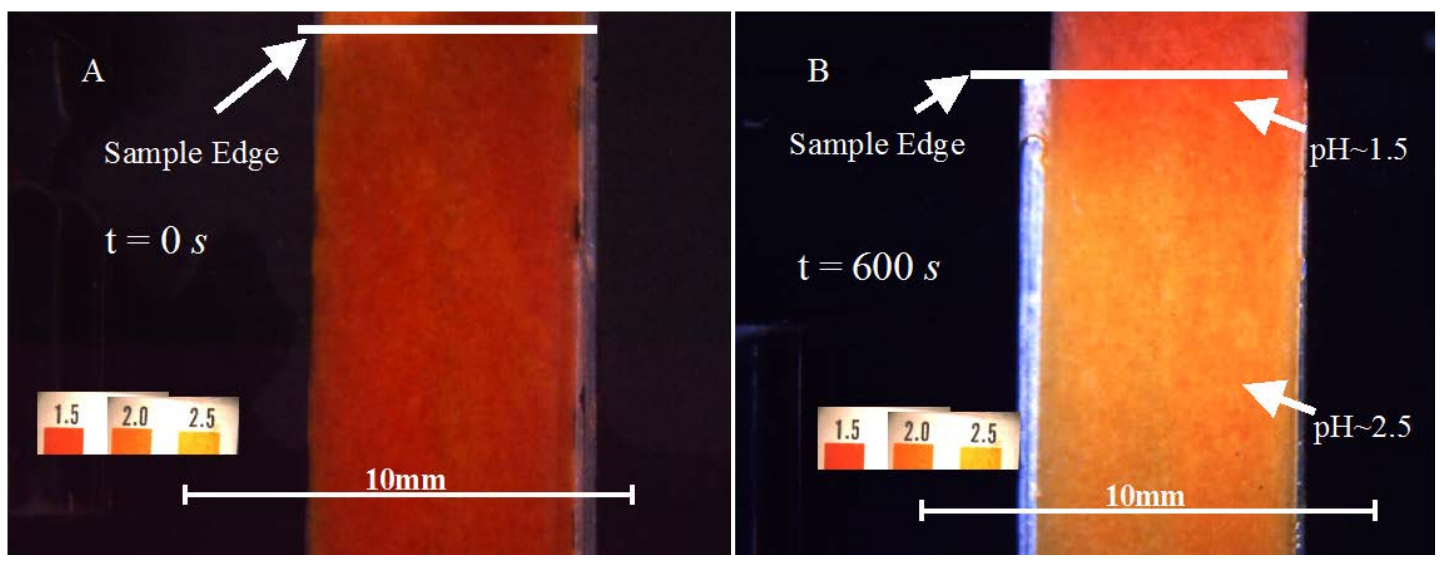

Figure 32: Variation in $\mathrm{pH}$ with time. A) At $t=0 \mathrm{~s}$ the $\mathrm{pH}$ is approximately 1.5 throughout the simulated IGC path. B) At $t=600 \mathrm{~s}$, only a small region (approximately $1.5 \mathrm{~mm}$ from the free surface) is at $\mathrm{pH}=1.50$. The remainder of the simulated IGC path is at $\mathrm{pH}>2.50$.

The $p H$ within about $1.5 \mathrm{~mm}$ of the free surface appeared to remain relatively constant at $p H \approx 1.50$. The $p H$ appeared to increase to value of 2.5-3.0 within $2.2 \mathrm{~mm}$ of the free surface. These results seem to demonstrate that there is a $\mathrm{pH}$ gradient within an IGC path in AA6005A-T6. Also note that the color of the litmus paper after the experiment (Figure 32B) is uniform across its width, and is not constrained to the shallow channel milled along the center of $5.5 \mathrm{~mm}$ groove. This is due to the solution wicking toward the outer edges of the litmus paper during the experiment.

The results presented in Figure 32 are similar to what has been observed in other wrought aluminum alloy systems, in that the $\mathrm{pH}$ of the local environment within a corrosion path is independent of the $p H$ of the bulk environment $[38,39]$. In contrast to the results presented in Figure 32, however, most studies of the local environment within a corrosion path of wrought aluminum alloys have been 
conducted with the bulk solution at a near-neutral $p H$. In these studies, the local $p H$ within a corrosion path was found to be lower $(\sim 3.5)$ than that of the near-neutral bulk solution.

The effect of diffusion path length on the local $p H$ was evaluated in a more quantitative manner using the sample configuration illustrated in Figure 33. Small ( $0.45 \mathrm{~mm}$ diameter) holes were drilled through the thickness of a sample of AA6005A-T6 extrusion to simulate an IGC path. The sample was then milled into a wedge, such that the final length of the holes ranged from $1.2 \mathrm{~mm}$ to $2.67 \mathrm{~mm}$. A small circle of filter paper was place atop each of the holes, and the bottom of the sample was exposed to a solution of aerated $3.5 \% \mathrm{NaCl}$ at $1.0<p H<5.5$. The solution filled each of the holes by capillary forces. The sample was allowed to equilibrate for 30 minutes, and the $p H$ atop each of the simulated IGC paths was measured using a $p H$ microelectrode. Note that these results give the $p H$ at the tip of a simulated IGC path of a given length, and do not give any direct information about the $\mathrm{pH}$ along the length of a given corrosion path. The results of these experiments are summarized in Figure 34. 

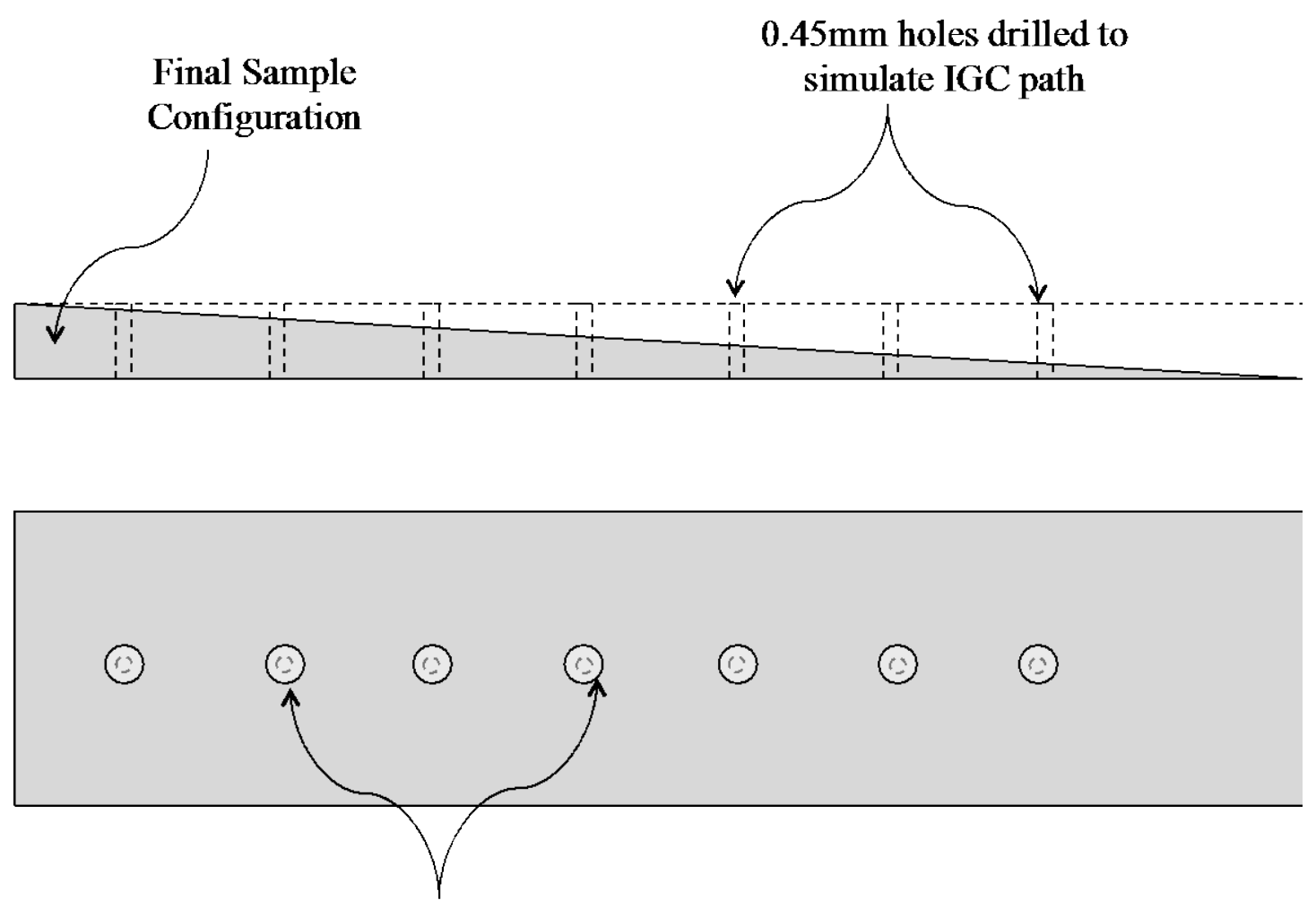

Small discs of filter paper placed atop each of the simulated IGC paths

Figure 33: Sample configuration used to determine the local pH at varied IGC path lengths. IGC paths were simulated by drilling $0.45 \mathrm{~mm}$ diameter holes into a wedge-shaped sample of AA6005A-T6. The pH as a function of IGC path length was determined by measuring the $\mathrm{pH}$ of solution on a small piece of filter paper placed atop each of the simulated IGC paths with a microelectrode. 


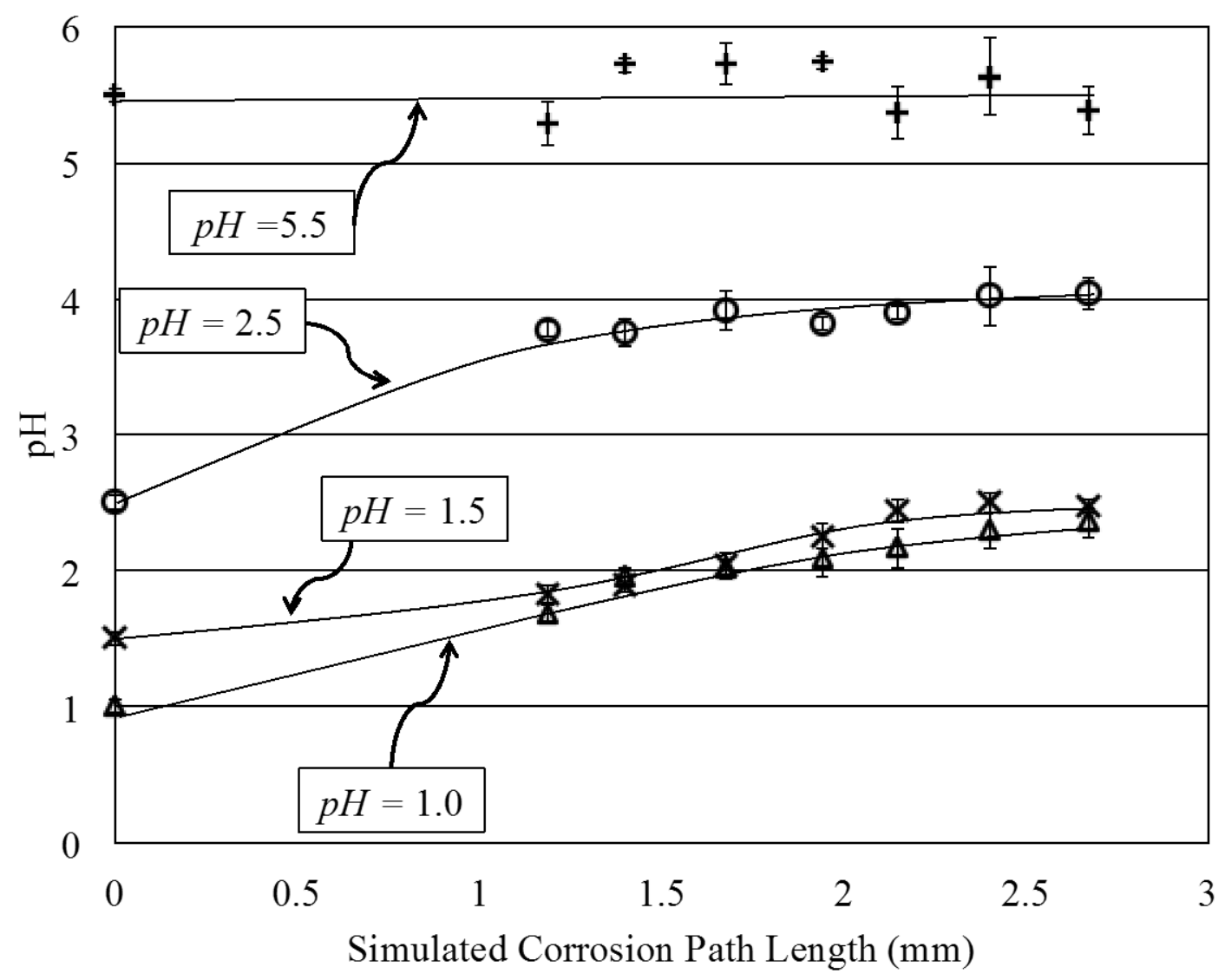

Figure 34: Effect of corrosion path length and acidity of bulk environment on the $\mathrm{pH}$ of the local environment within a simulated corrosion path. The $\mathrm{pH}$ within an occluded region increases to a value of approximately 2.50 when the external $\mathrm{pH} \leq 1.50$. The $\mathrm{pH}$ within an occluded region increases to approximately 4.0 when the bulk $\mathrm{pH}=2.50$. The $\mathrm{pH}$ within an occluded region is similar to that of the bulk solution when the external $\mathrm{pH}=5.50$.

When the $p H$ of the bulk solution was 1.0, the $p H$ at the tip of a simulated corrosion path increased approximately linearly to a value of around 2.3 as the simulated corrosion path length approached $2.7 \mathrm{~mm}$. When the $p H$ of the bulk solution was increased to 1.5 , the $p H$ at the tip of a simulated corrosion path increased to a maximum value of 2.5 when the simulated corrosion path was $\geq 2.3 \mathrm{~mm}$. 
Increasing the $p H$ of the bulk solution to 2.5 caused the $p H$ at the tip of a simulated corrosion path to increase to 4.0 when the corrosion path length exceeded $1.7 \mathrm{~mm}$. The $p H$ at the tip of a simulated corrosion path was similar to that of the bulk environment when the $p H$ of the external environment was at 5.50. The results illustrated in Figure 34 agree reasonably well with the qualitative results presented in Figure 32, and give valuable insight into the decrease in IGC velocity with increasing penetration depth when the material is exposed to a low- $\mathrm{pH}$ aqueous environment.

The effects of corrosion path length on the local $p H$ at the tip of a simulated corrosion path when the bulk $p H$ was held at 1.5 were considered in developing a model to explain the decay in IGC velocity with increasing depth of corrosion attack. Equation 7 is an linear fit to the data in Figure 34 for a $p H=1.50$ solution. This equation gives the relationship between $p H$ at the tip of a corrosion path and corrosion path length, $L$, when AA6005A-T6 is exposed to an aerated saltwater solution at $p H=1.5$. Equation 7 is only valid for $L \leq 2.3 \mathrm{~mm}$ since the $p H$ becomes essentially constant at 2.5 at $L>2.3 \mathrm{~mm}$.

\section{Equation 7}

$$
p H=0.435 L+1.5(L \text { is in } \mathrm{mm})
$$

It is not likely that the observed increase in local $p H$ with increasing corrosion path length was due to a lack of availability of $H^{+}$at the tip of the corrosion path, as there is an abundance of $H^{+}$in the bulk environment (low $p H$ ), and $H^{+}$diffuses rapidly in saltwater solutions $[66,67]$. It is also unlikely that the observed increase in local $\mathrm{pH}$ with increasing corrosion path length is due to a decrease in dissolved $\mathrm{O}_{2}$ content near the tip of a corrosion path, as decreased $\left[\mathrm{O}_{2}\right]$ generally results in a local 
decrease in solution $p H$. The relationship between $p H$ and $\left[A l^{3+}\right]$ at the tip of an IGC path was therefore examined, and used to develop a model relating $A l^{3+}$ diffusion and IGC velocity in AA6005A-T6 extrusions.

Several reports have demonstrated that the $p H$ within a corrosion path in certain $\mathrm{Al}$ alloys is ultimately controlled by precipitation of specific solid compounds in the corrosion path $[39,40,43,44]$. The precipitates controlling the equilibrium $p H$ within a corrosion path of an $\mathrm{Al}$ alloy could be $\mathrm{Al}(\mathrm{OH})_{3}$ or some form of $\mathrm{Al}_{2} \mathrm{O}_{3}$ [43, $44,68]$. According to these reports, when the solubility product $\left[\mathrm{Al}^{3+}\right][\mathrm{OH}]^{3}$ reaches the solubility constant for a specific solid compound, $K^{S P}$, precipitation occurs, and the $p H$ within the corrosion path is stabilized at a value corresponding to the equilibrium value of $\left[\mathrm{OH}^{-}\right]$. The values of $K^{S P}$ for several solid compounds are given in Table 7. Note that all values of $K^{S P}$ in Table 7 are expressed in terms of the product $\left[\mathrm{Al}^{3+}\right][\mathrm{OH}]^{3}$. In his discussion of the stability of $\mathrm{Al}_{2} \mathrm{O}_{3}$, Pourbaix indicated that aluminum hydroxide gel $\left(\mathrm{Al}(\mathrm{OH})_{3}\right)$ existing in a corrosion path is often unstable, and eventually crystallizes to form a hydrated phase of $\mathrm{Al}_{2} \mathrm{O}_{3}$ [68]. Pourbaix stated the product $\left[\mathrm{Al}^{3+}\right][\mathrm{OH}]^{3}$ can be used as a valid approximation to determine the solubility constant for various forms of $\mathrm{Al}_{2} \mathrm{O}_{3}$ since $\mathrm{Al}(\mathrm{OH})_{3}$ is a precursor to the formation of $\mathrm{Al}_{2} \mathrm{O}_{3}$. 
Table 7: Solubility products for several solid species that may control the $\mathrm{pH}$ within an IGC path in AA6005A-T6 [68]

\begin{tabular}{|c|c|c|}
\hline Name & Chemical Formula & $\boldsymbol{K}^{\boldsymbol{S P}},\left[\mathbf{A l}^{3+} \boldsymbol{]}\left[\mathbf{O H} \boldsymbol{H}^{\mathbf{3}}\right.\right.$ \\
\hline Hydrargillite & $\mathrm{Al}_{2} \mathrm{O}_{3} \cdot 3 \mathrm{H}_{2} \mathrm{O}$ & $5.01 \times 10^{-37}$ \\
\hline Bayerite & $\mathrm{Al}_{2} \mathrm{O}_{3} \cdot 3 \mathrm{H}_{2} \mathrm{O}$ & $3.16 \times 10^{-36}$ \\
\hline Böhmite & $\gamma-\mathrm{Al}_{2} \mathrm{O}_{3} \cdot 3 \mathrm{H}_{2} \mathrm{O}$ & $1.00 \times 10^{-34}$ \\
\hline Corundum & $\alpha-\mathrm{Al}_{2} \mathrm{O}_{3}$ & $3.98 \times 10^{-34}$ \\
\hline Aluminum Hydroxide & $\mathrm{Al} \mathrm{OH})_{3}$ & $5.01 \times 10^{-33}$ \\
\hline
\end{tabular}

Assuming the $p H$ at the tip of an IGC path in AA6005A-T6 is controlled by the precipitation of some solid compound when the alloy is exposed to a low- $p H$ saltwater solution, $\left[A l^{3+}\right]$ at the tip of the IGC path can be estimated as:

Equation 8

$$
\left[A l^{3+}\right]=\frac{K^{S P}}{\left[O H^{-}\right]^{3}}
$$

Where $\left[\mathrm{OH}^{-}\right]$is related to the $\mathrm{pH}$ at the tip of the IGC path (Equation 7), and $\mathrm{K}^{S P}$ is the solubility constant for some solid compound in equilibrium with $A l^{3+}$ (from Table 7). An expression for $\left[\mathrm{Al}^{3+}\right]$ at the tip of a corrosion path in terms of corrosion path length can be found using Equation 7 and applying the relationship $\left[\mathrm{OH}^{-}\right]\left[\mathrm{H}^{+}\right]=10^{-14}$.

\section{Equation 9}

$$
\left[A l^{3+}\right]=\frac{K^{S P}}{(-\log (14-(0.435 L+1.5)))^{3}}
$$

With the approximate value of $\left[A l^{3+}\right]$ at the tip of an IGC path known, molar flux of $A l^{3+}$ from the tip of an IGC path can be estimated by using a one-dimensional application of Fick's first law:

Equation 10

$$
J_{A l 3+}=-D_{A l 3+} \frac{d C_{A l 3+}}{d x}
$$


Where $J_{A l 3+}$ is the molar flux of $\mathrm{Al}^{3+}$ (in $\mathrm{mol} /\left(\right.$ area $\times$ time),$D_{A l 3+}$ is the diffusion

coefficient of $A l^{3+}$ in the electrolyte, $d C_{A l 3+}$ is the difference between $\left[A l^{3+}\right]$ at the tip of the IGC path and $\left[\mathrm{Al}^{3+}\right]$ in the external environment, and $d x$ is the diffusion path length. Assuming that $A l^{3+}$ diffuses exclusively along an IGC path, $d x$ can be estimated as the structure-corrected corrosion path length, $L$.

The molar flux of $A l^{3+}$ can be used to calculate an IGC velocity, supposing that IGC penetration is limited by diffusion of $A l^{3+}$ away from the tip of an IGC path. First, assume that the IGC path is a straight-walled rectangular tube with cross sectional area $A$ (Figure 35). In order to advance the IGC path a distance $d L$, the volume of $A l$ that must be dissolved is $A d L$. Multiplying this volume by the molar volume of $\mathrm{Al}\left(\mathrm{MV} V_{A l}, 0.1 \mathrm{~mol} / \mathrm{cm}^{3}\right)$ gives the total number of moles that must be dissolved to advance the IGC path the distance $d L$. The dissolution rate within the volume $A d L$ would then be:

Equation 11

$$
\varphi=M V_{A l} \frac{A d L}{d t}
$$

Where $\varphi$ is the dissolution rate (in mol/time), and $d t$ is the increment of time required to dissolve the volume $A d L$. Multiplying the molar flux of $A l^{3+}$ (Equation 10) by the cross sectional area of the IGC path, $A$, gives the rate at which $A l^{3+}$ ions are migrating through a plane a short distance from the tip of the IGC path, $Z$ (Equation 12). The values of $Z$ and $\varphi$ must be equal in order to maintain quasi steady-state onedimensional flux (Equation 13). 
Equation 12

$$
Z=J_{A l 3+} \times A=-D_{A l 3+} A \frac{d C_{A l 3+}}{L}
$$

Equation 13

$$
Z=\varphi=-D_{A l 3+} A \frac{d C_{A l 3+}}{L}=M V_{A l} \frac{A d L}{d t}
$$

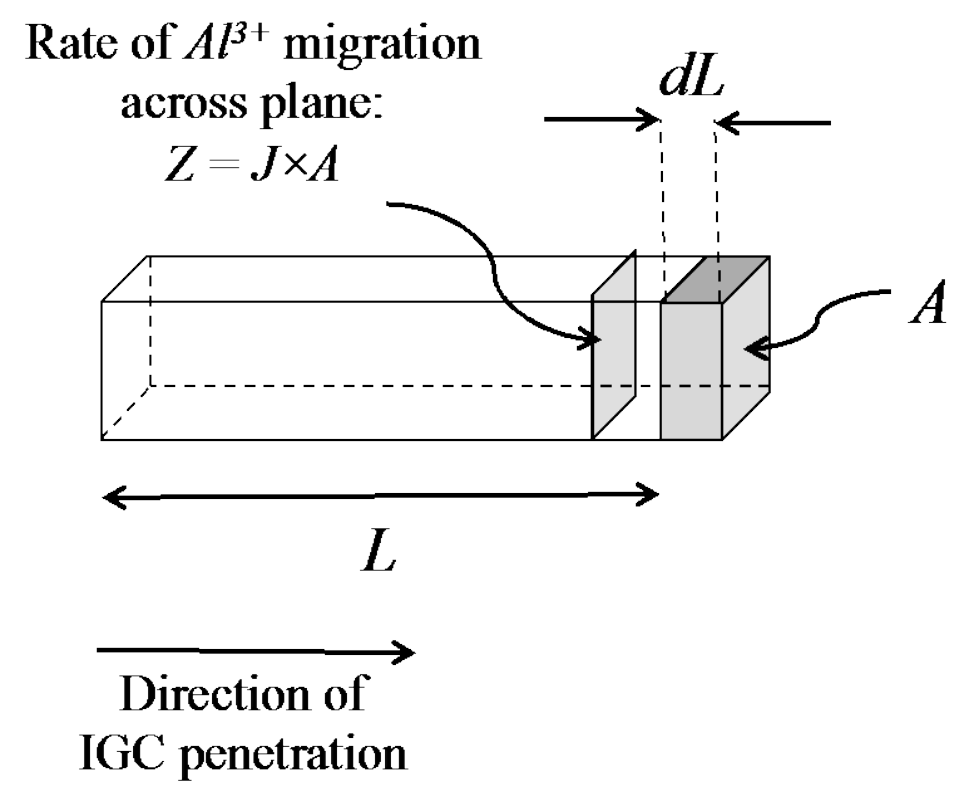

Figure 35: Model of IGC path used to determine IGC velocity. The IGC path was assumed to be a straight-walled rectangular tube with cross sectional area $A$. The IGC velocity was related to the molar flux by considering the conditions necessary to dissolve a volume of the IGC path with a length $d L$.

By rearranging Equation 13, the value of $d L / d t$ (which is equivalent to IGC velocity) can be determined. Notice that the cross sectional area of the IGC path, $A$, cancels when solving for $d L / d t$, indicating that IGC velocity is independent of the size and shape of the IGC path that we have assumed in Figure 35. Inserting the 
expression for $d C_{A l 3+}$ (Equation 9), and applying known values of $D_{A l 3+}\left(4.2 \times 10^{-6}\right.$ $\left.\mathrm{cm}^{2} / \mathrm{s},[67]\right)$ and $M V_{A l}\left(0.1 \mathrm{~mol} / \mathrm{cm}^{3}\right)$, gives an expression for IGC velocity with varying corrosion path length (Equation 14).

Equation 14

$$
V_{I G C}(L)=\frac{-D_{A l 3+}}{M V_{A l}}\left[\frac{K^{S P}}{(-\log (14-(0.435 L+1.5)))^{3} L}\right]
$$

The diffusion-based model was tested by plotting $V_{I G C}$ vs. $L$ curves (as predicted by Equation 14) using different values of $K^{S P}$, and comparing the experimental $V_{I G C}$ vs. $L$ data (Figure 36). The different values of $K^{S P}$ used in testing Equation 14 corresponded to the solubility constants for the different solid species that may be controlling the $p H$ within an IGC path of AA6005A-T6 (Table 7). 


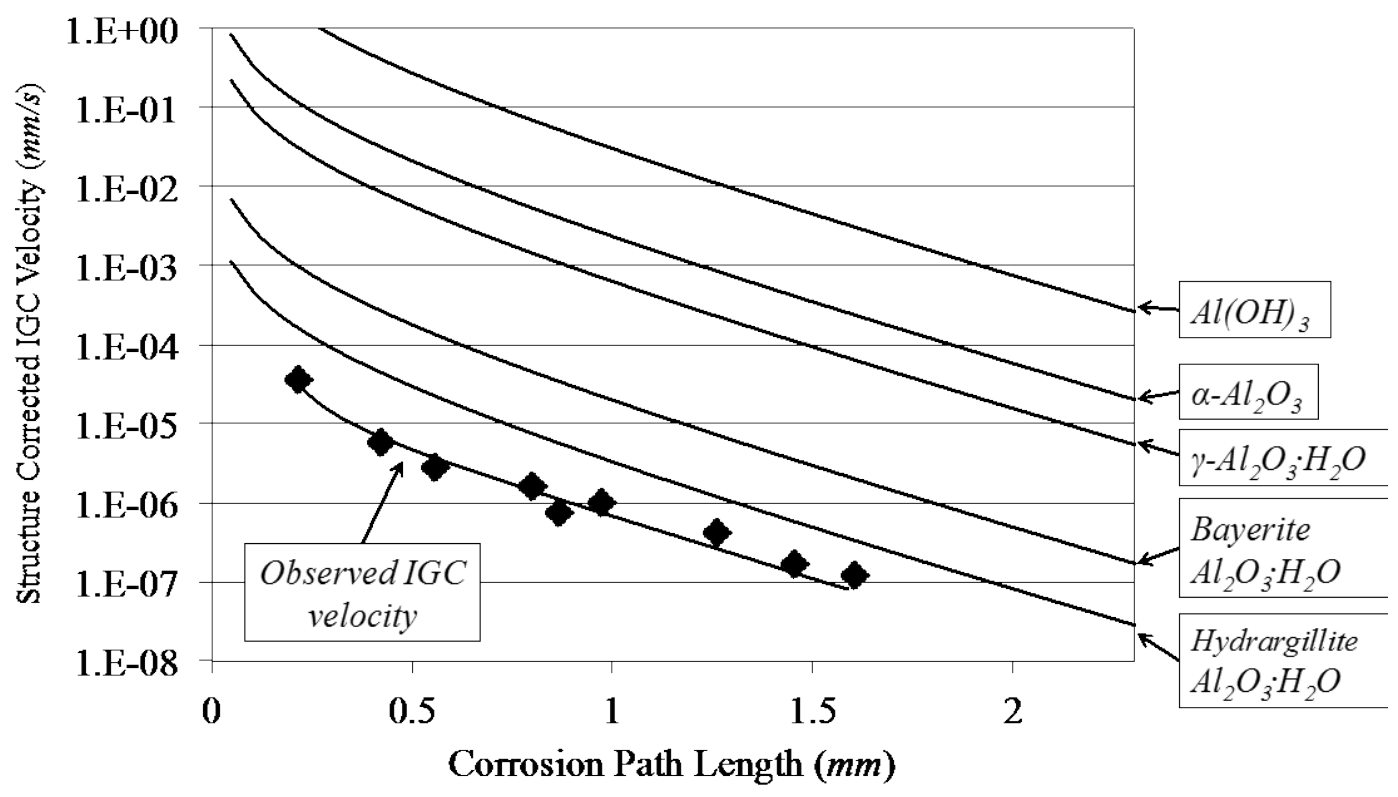

Figure 36: Test of diffusion-based model to predict IGC velocity in AA6005A-T6. The model seems to accurately model the observed decay in IGC velocity with increasing corrosion path length. However, the observed IGC velocity was somewhat lower than the predicted values of IGC velocity.

The general shape of the $V_{I G C}$ vs. $L$ curves predicted by Equation 14 closely resembles the experimentally observed decay in $V_{I G C}$ with increasing corrosion path length. The good agreement in trend between the predicted and experimental $V_{I G C}$ behavior suggests that $V_{I G C}$ in AA6005A-T6 exposed to a low- $p H$ saltwater solution is ultimately limited by the ability to transport $A l^{3+}$ from the tip of an IGC path into the external environment. This implies that, at least for the range of corrosion path lengths explored here, the mechanisms of electrochemical dissolution within an IGC path (anodic and cathodic reactions) do not change with increasing corrosion path length. 
Though the diffusion-based model seems to capture the general trend of $V_{I G C}$ decay in AA6005A-T6, the observed $V_{I G C}$ is slower than the predicted $V_{I G C}$ for all values of $K^{S P}$ in Table 7. There are several possible explanations for this observed discrepancy. First, it is possible that there may be some other solid compound that controls the $\mathrm{pH}$ within an IGC path in AA6005A-T6 that was not considered in this work. There are several forms of $\mathrm{Al}_{2} \mathrm{O}_{3}$ (such as $\theta-\mathrm{Al}_{2} \mathrm{O}_{3}$ and $\eta-\mathrm{Al}_{2} \mathrm{O}_{3}$ ) for which the solubility constant is unknown. It is possible that the solubility constant for some solid compound not considered in this work is much lower, thereby making the predicted and observed values of $V_{I G C}$ agree more precisely.

The difference between predicted and observed values of $V_{I G C}$ may also be explained by addressing a key assumption used in developing the diffusion-based model. It was assumed that the $\left[\mathrm{Al}^{3+}\right]$ gradient is linear within the corrosion path with $\left[A l^{3+}\right]$ being greatest at the tip of the corrosion path and negligible in the bulk environment. With this assumption, the model predicts that $A l^{3+}$ would migrate away from the tip of an IGC path in a unidirectional manner. It is not likely that the $\left[\mathrm{Al}^{3+}\right]$ gradient is perfectly linear within a corrosion path. Variations from linearity in the $\left[\mathrm{Al}^{3+}\right]$ gradient within the corrosion may be caused by a number of factors (such as IGC branching, dissolution along the IGC path, etc.) not captured with the model. Therefore it is likely that $A l^{3+}$ can migrate toward the tip of the IGC path to some degree. Adjusting the model to account for possible "two-way" diffusion of $A l^{3+}$ would tend to decrease the predicted $V_{I G C}$, thereby giving better agreement between the predicted and observed $V_{I G C}$. 


\subsection{Qualitative measurement of SCC susceptibility in AA6005A Extrusions}

\subsubsection{Experimental Methods - Qualitative SCC of AA6005A}

The effects of heat treatment and environment on SCC susceptibility of AA6005A extrusions were qualitatively examined by conducting breaking load tests (BLT) [69]. Breaking load tests involve applying a known tensile stress to a sample while exposing the sample to known environment for a prescribed period of time. In BLT, test samples are exposed to a corrosive environment with an applied stress, and control samples are exposed to a corrosive environment without an applied stress. After the samples have been stressed and exposed for the prescribed amount of time, the combined effects of applied stress and exposure are evaluated by measuring the residual strength of the sample after the exposure. The residual strength of a sample in BLT corresponds to the ultimate tensile stress (UTS) after exposure, which is measured by performing a standard monotonic tensile stress. The value of residual strength is then compared to the UTS of a sample that was exposed to the test solution without an applied stress. The residual strength of samples after BLT is reported as a percentage of retained UTS vs. the UTS of samples that were exposed with no applied stress. A high percentage of retained strength corresponds to good SCC resistance, and a low percentage of retained strength corresponds to poor SCC 
resistance. Breaking load tests have served as a relatively rapid assessment of SCC susceptibility in a number of $A l$ alloys $[58,70,71]$.

Tensile loads ranging from $0.7 \sigma_{y}$ to $1.0 \sigma_{y}\left(\sigma_{y}=\right.$ the yield strength of the alloy) were applied along direction $\mathbf{3}$ to modified sub-sized tensile bars using calibrated proof rings. The sample geometry is illustrated in Figure 37.The proof rings were designed to be compliant so that the applied load would remain nearly constant over the duration of the experiments, even if the samples elongated slightly. The displacement of the proof rings was periodically measured to monitor the applied load. The experimental setup is illustrated in Figure 38.

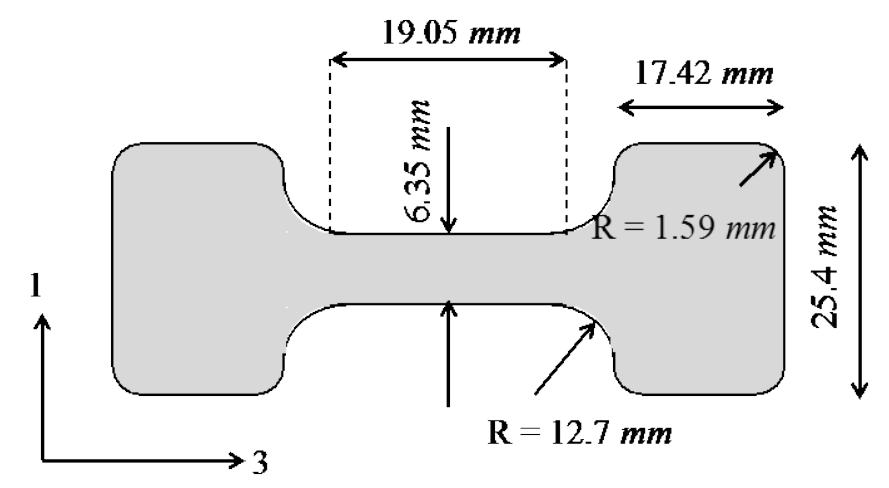

Figure 37: Modified sub-sized tensile bar geometry used for BLT. 


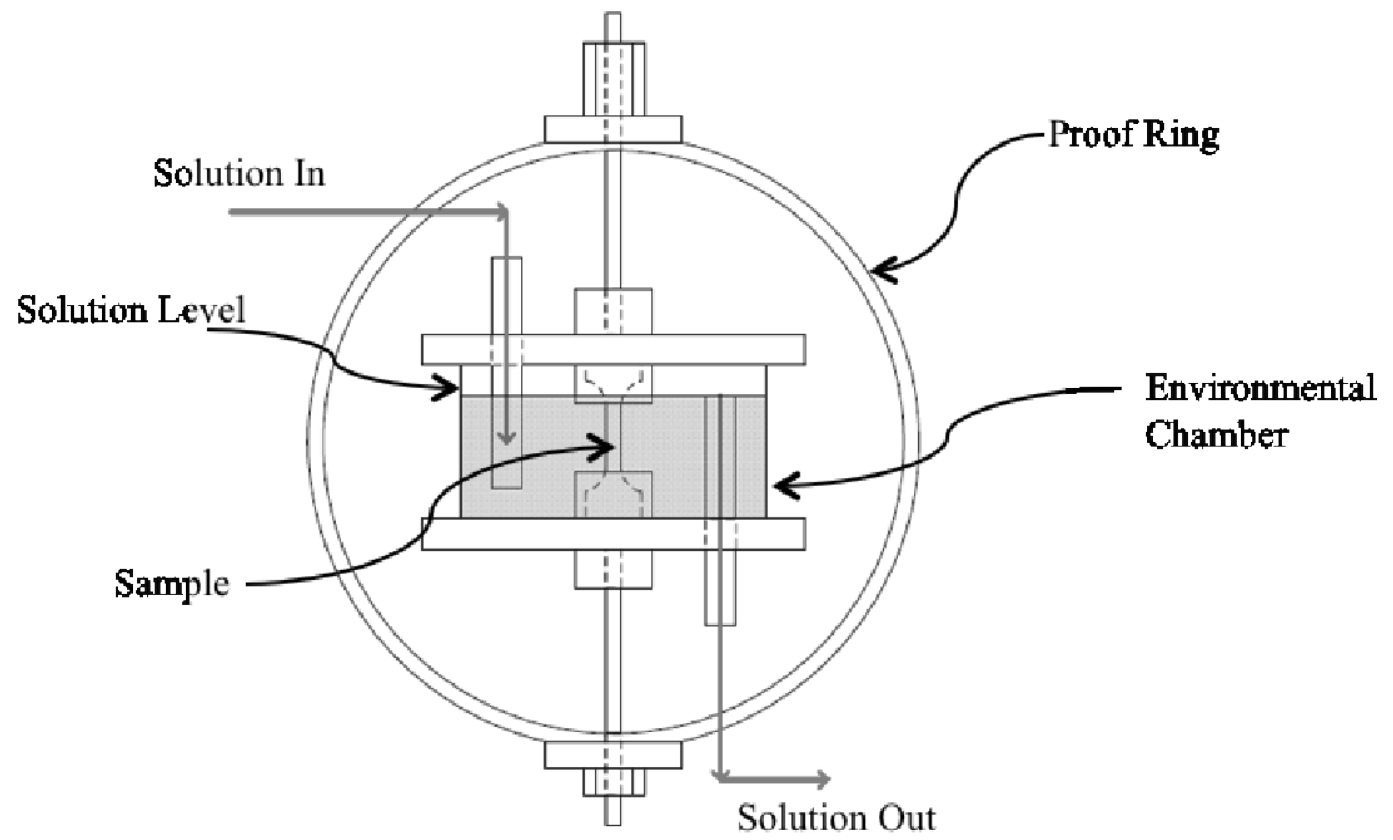

Figure 38: Experimental setup for conducting breaking load tests. A static tensile load was applied to the sample using a calibrated proof ring, and the aqueous environment was circulated through environmental chambers using a gravity fed system.

For this work, samples having a T4, T6, or OA temper were tested in aerated solutions of $3.5 \% \mathrm{NaCl}$ with $0.5<p H<2.0$. Samples were also exposed to solutions containing $0.3 \% \mathrm{As}_{2} \mathrm{O}_{3}$ to qualitatively examine the effects of a hydrogen recombination poison (HRP) on SCC susceptibility of AA6005A extrusions. The test solutions were circulated through environmental chambers using a gravity-fed system (Figure 39). The primary reservoir contained $15 L$ of solution. The solution was aerated by bubbling laboratory air through the solution in the primary reservoir. Each of the environmental chambers contained $\sim 1 L$ of solution, and the flow-rate in each of the chambers was held constant at $0.5 \mathrm{~L} / \mathrm{min}$. The grip material was AA6005A to 
prevent galvanic effects between the samples and grips. The exposure time for all samples was $200 \mathrm{hr}$.

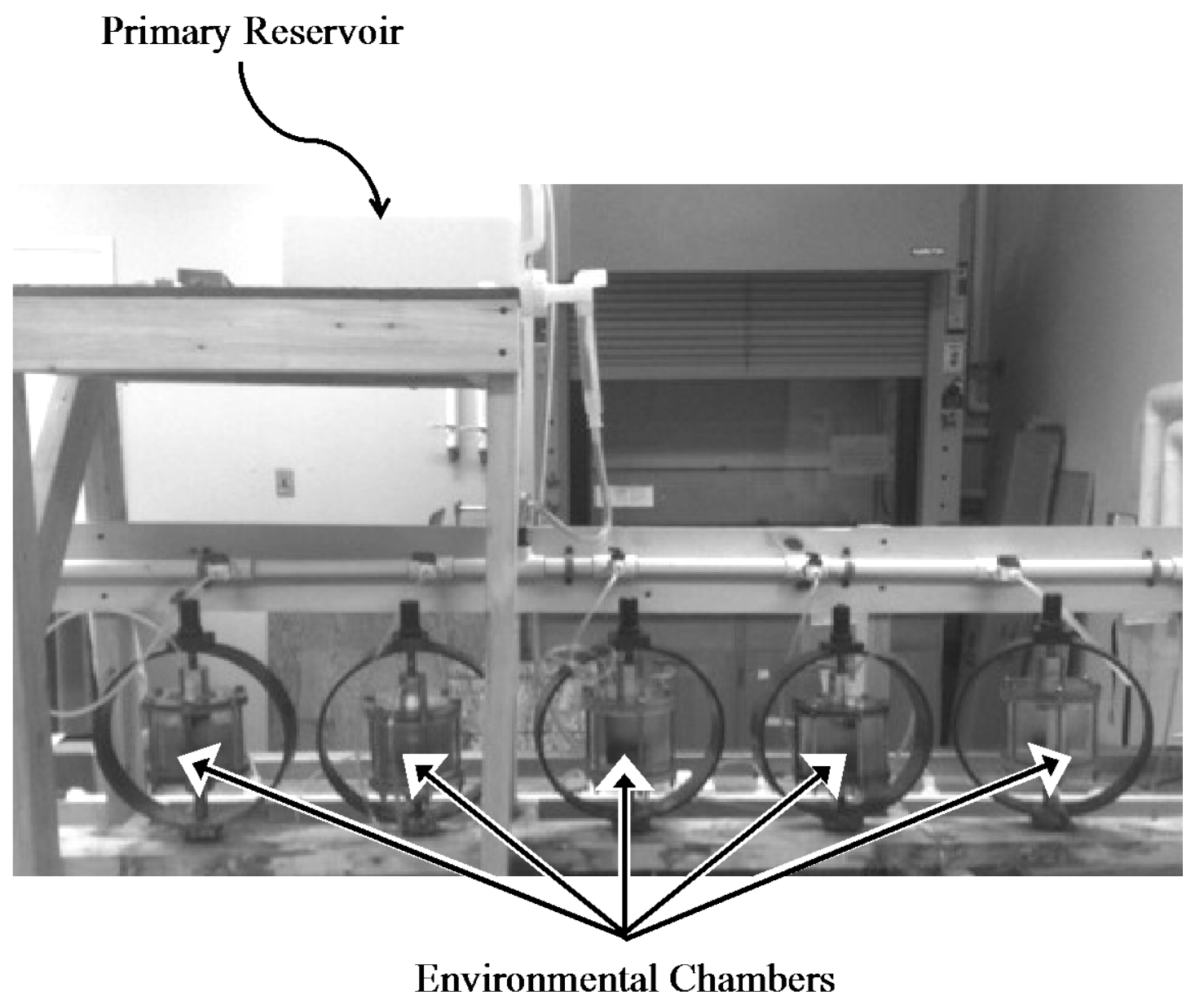

Figure 39: Gravity fed system for conducting qualitative SCC analysis of AA6005A extrusions.

\subsubsection{Results and Discussion - Qualitative SCC of AA6005A}

The effects of heat treatment on the residual strength of AA6005A extrusions exposed to a solution of $3.5 \% \mathrm{NaCl}$ at $\mathrm{pH}=1.00$ are illustrated in Figure 40. The error bars correspond to the observed standard deviation for each sample condition. A 
modest decrease in residual strength with increasing exposure load was observed when the samples were in a T6 and OA temper. The combined effects of an applied load and exposure to the corrosive environment had a negligible influence on the residual strength of samples tested in a T4 temper. These results suggest that AA6005A-T6 and AA6005A-OA may be mildly susceptible to SCC when exposed to a low- $p H$ aqueous saltwater environment with large applied tensile loads.

The magnitude of the stresses applied to the samples in these experiments was much larger than the maximum design stress for the CSPP solar frames (See section 0). The solutions used in these experiments was also much more aggressive than a typical industrial marine environment. Since there only a minor $(\sim 15 \%)$ decrease in residual strength was observed in these experiments, it seems that AA6005A extrusions will be suitable for use in CSPP frames in a typical industrial marine environment. 


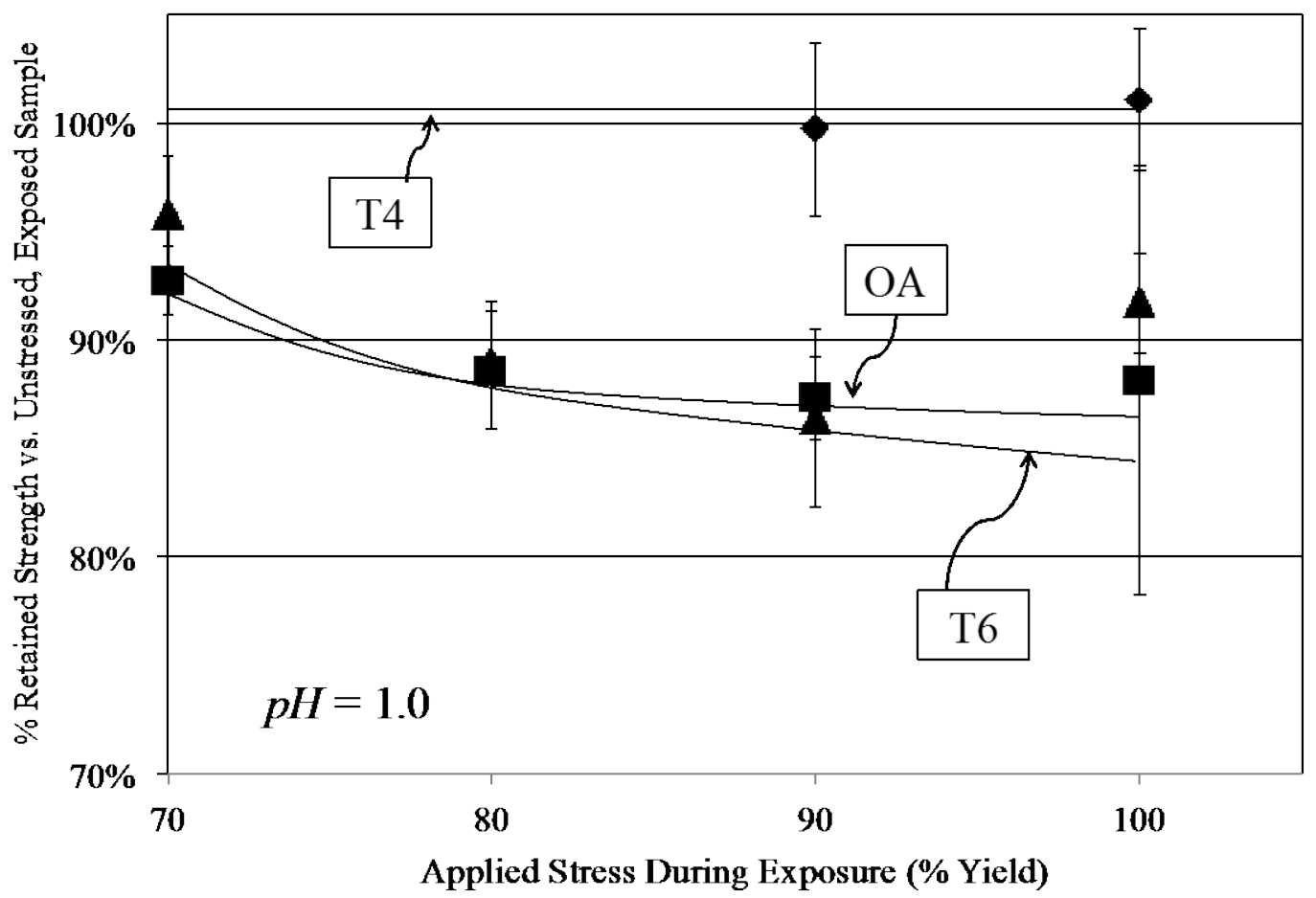

Figure 40: Effect of heat treatment on the residual strength of AA6005A extrusions exposed to $3.5 \% \mathrm{NaCl}$ at $\mathrm{pH}=1.0$. Increasing the applied load during exposure tended to decrease the residual strength in samples tested in a T6 (घ) and $O A(\mathbf{\Delta})$ temper. Negligible decrease in residual strength was observed when the samples were tested in a T4 $(\bullet)$ temper. These results suggest AA6005A may be susceptible to SCC when in a T6 and OA temper.

Figure 41 compares the combined effects of exposure and applied stress to the effects of exposure alone for AA6005A-T6 samples exposed to acidified saltwater with $p H$ ranging from 0.5 to 2.0 . The applied stress for the loaded samples was $100 \%$ $\sigma_{y}(250 \mathrm{MPa})$. The combined effects of load and exposure did not result in a decrease in residual strength when $p H=0.50$, suggesting that bulk dissolution of the material was the dominant factor in determining the loss in strength in this environment. A 
small, but statistically significant decrease in residual strength between the loaded and unloaded samples was observed when the material was exposed to a solution with $p H$ $=1.00$, suggesting that AA6005A-T6 is most susceptible to $\mathrm{SCC}$ in these conditions. When the $p H$ was raised to a value of 2.00 , an insignificant decrease in residual strength was observed. These results indicate that AA6005A-T6 may be mildly susceptible to $\mathrm{SCC}$ in $3.5 \% \mathrm{NaCl}$ when $1.00 \leq p H<2.00$.

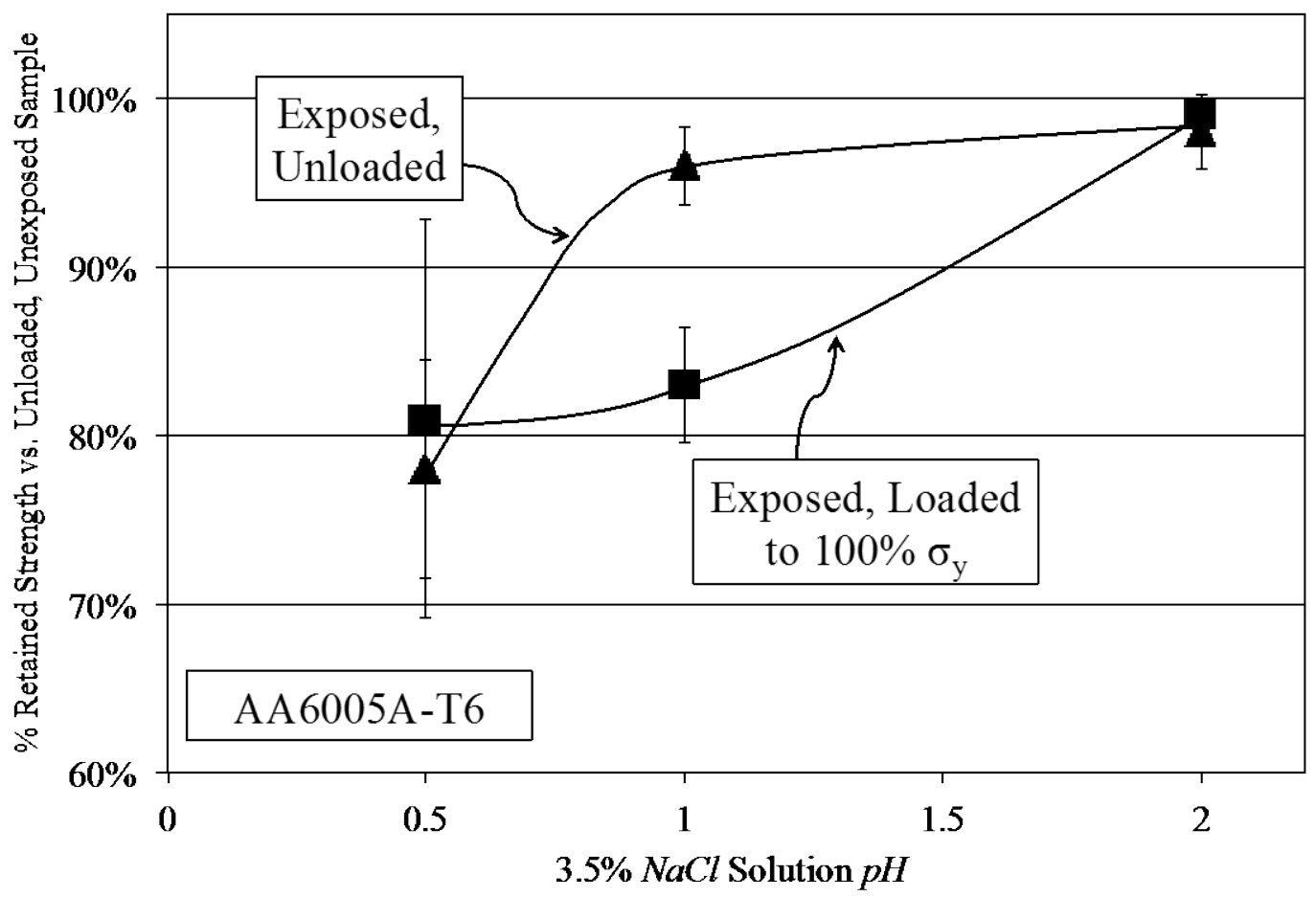

Figure 41: Effect of pH on SCC susceptibility in AA6005A-T6. The combined effects of exposure and applied load did not decrease the residual strength when $p H=0.50$. The combined effects of load and exposure resulted in a decrease in residual strength when $p H=1.00$. A negligible decrease in residual strength was observed when $p H=2.00$. These results indicate that AA6005A-T6 may be susceptible to SCC when $1.00 \leq p H<2.00$ 
The presence of an HRP in a solution containing $3.5 \% \mathrm{NaCl}$ at $\mathrm{pH}=1.00$ actually improved the residual strength of AA6005A-T6 (Figure 42). These results suggest that $\mathrm{As}_{2} \mathrm{O}_{3}$ effectively slows the cathodic reaction to decrease the rate of dissolution. Also, since $\mathrm{As}_{2} \mathrm{O}_{3}$ would tend to magnify the effects of hydrogen absorption during exposure, these results suggest that hydrogen embrittlement is likely not the primary operating mechanism of SCC in AA6005A. Experiments designed to quantify the effects of a hydrogen recombination poison on the SCC susceptibility of AA6005A-T6 are summarized in section 4.5.2.

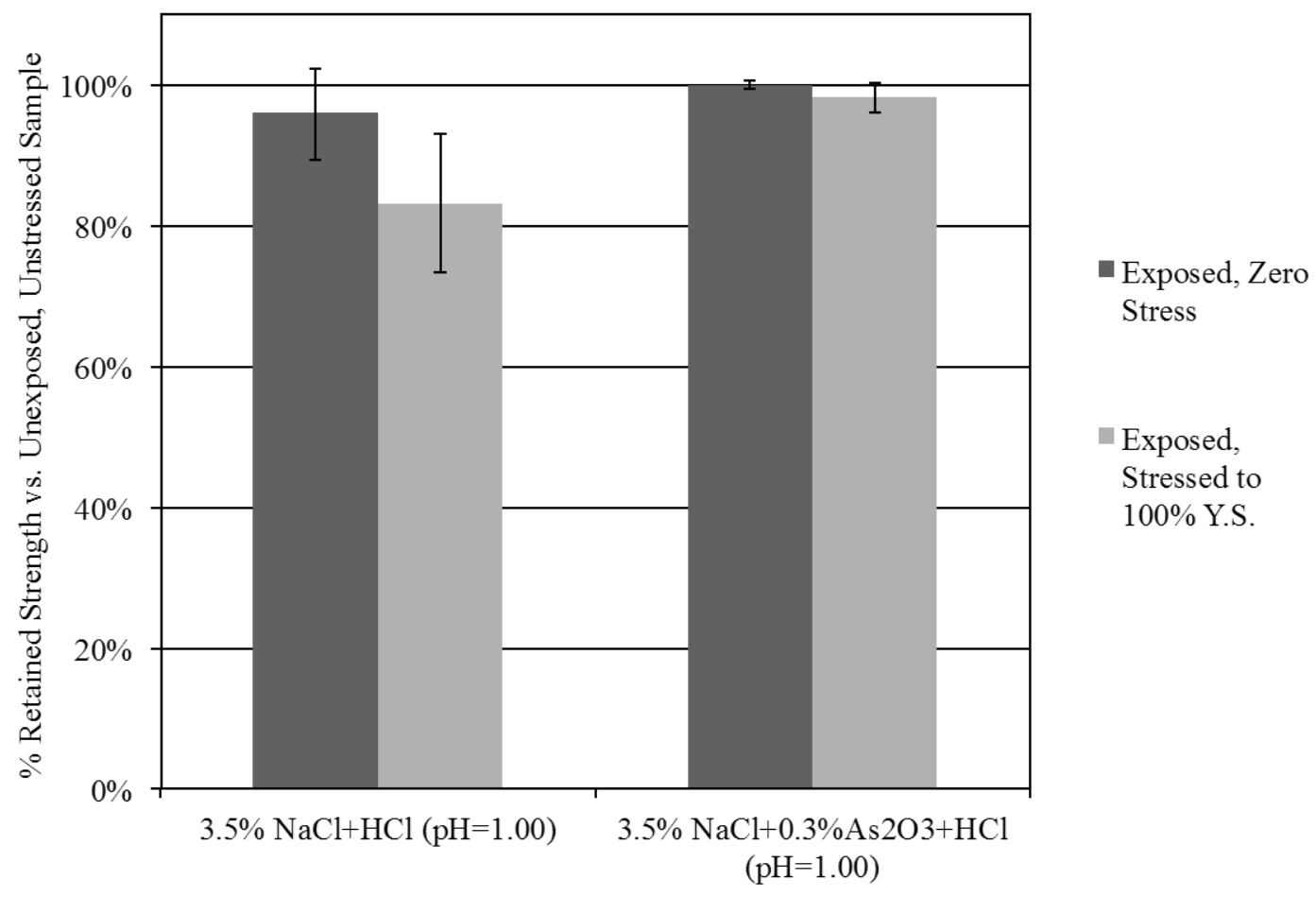

Figure 42: Effect of a hydrogen recombination poison on SCC susceptibility of AA6005A-T6. A minimal decrease in residual strength was observed with the presence of $\mathrm{As}^{3+}$ in the test environment. These results suggest that hydrogen embrittlement is not an operating mechanism of SCC in AA6005A 


\subsection{Quantitative measurement of SCC in AA6005A Extrusions}

Although the results from a qualitative assessment of SCC in AA6005A using breaking load tests indicated that the material was only mildly susceptible to SCC, additional experiments to quantify the crack growth behavior of AA6005A-T6 extrusions exposed to an aggressive aqueous environment were conducted

\subsubsection{Experimental Methods - Quantitative SCC of AA6005A}

Crack growth rate (SCC-CGR) studies were performed on fatigue pre-cracked compact tensile bars of AA6005A-T6. The value of $K_{I C}$ for the sample configuration used in this work was $30.68+/-0.78 M P a \sqrt{\mathrm{m}}$, as measured by conducting fracture toughness tests of the samples in an ambient environment. The thickness of the extruded profiles used in this study were $3.125 \mathrm{~mm}$, which was not sufficient to satisfy plane strain conditions up to fracture, so the data presented here is applicable only to materials of similar thickness. Figure 43 illustrates the sample geometry of the compact tensile (CT) specimens. The CT specimens were prepared using wire electrical discharge machining (EDM). The wire diameter was $0.254 \mathrm{~mm}$. Wire EDM allowed the specimens to be produced without introducing residual stresses that could be present with conventional machining techniques.

The total crack length, $a$, prior to fatigue pre-cracking was $14.605 \mathrm{~mm}$. This initial value of crack length corresponds to the distance between the loading points and the end of the wire EDM notch. The width of the notches before fatigue precracking was $\sim 0.254 \mathrm{~mm}$ (approximate width of notch formed by wire EDM). A 
cyclic tensile load with an amplitude of $2225 N$ and load ratio $R=0.1$ was applied to the CT specimens to induce fatigue pre-cracks. The lengths of the fatigue pre-cracks were approximately equal to the thickness of the samples.

All samples were loaded along the $\mathbf{3}$ direction, and crack growth along direction 1 was measured for the SCC-CGR experiments. Most SCC-CGR measurements were conducted in aerated $3.5 \% \mathrm{NaCl}$ with $p H=1.50$. Some experiments were performed in an aerated solution of $3.5 \% \mathrm{NaCl}$ at $p H=2.0$. In addition, the possible significance of a hydrogen embrittlement mechanism was examined by adding $0.3 \% \mathrm{As}_{2} \mathrm{O}_{3}$ to a $\mathrm{pH}=1.50$ test solution for a few experiments. 


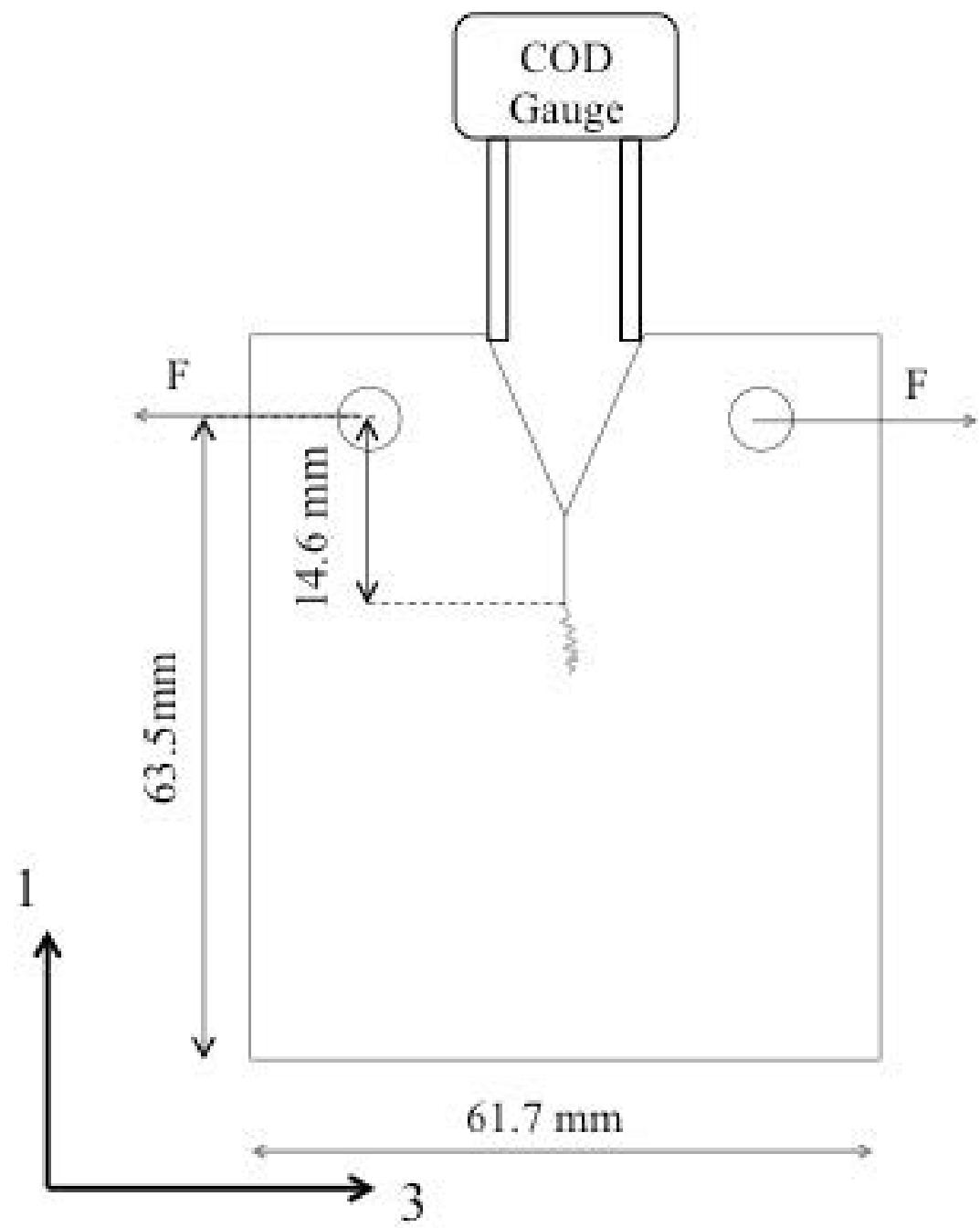

Figure 43: Sample geometry of CT specimens used for crack growth rate measurements. The COD gauge which was used to precisely measure the crack opening displacement, $d$, was mounted at the mouth of the CT specimen.

The samples were loaded using proof rings instrumented with strain gauges.

The strain gauges were mounted on the ID and OD of the proof rings at the 3 o'clock and 9 o'clock positions. Load $(F)$ vs. strain ( $\varepsilon$, as measured by strain gauges on proof rings) curves were constructed by applying a range of loads and recording the measured strain. The $F$ vs. $\varepsilon$ calibration was performed for each of the proof rings. 
Instrumenting the proof rings with strain gauges allowed the applied load, $F$, to be continually monitored throughout the duration of a SCC-CGR experiment using data acquisition equipment. The compact tensile specimens were instrumented with crack opening displacement (COD) gauges to precisely measure the crack opening displacement $(d)$ at the crack mouth.

Crack length $(a)$ was determined from the effective specimen modulus, $M_{E}$. The modulus is defined as:

\section{Equation 15}

$$
M_{E}=\frac{F}{d \times t}
$$

Where $t$ is the specimen thickness. As the crack length increased, the modulus of the sample decreased. The $M$ vs. $a$ relationship for the CT specimens used in this work was determined by performing modulus measurements on a sample of known thickness with crack lengths ranging from $14.605 \mathrm{~mm}$ to $23.85 \mathrm{~mm}$. The results of this calibration are shown in Figure 44. 


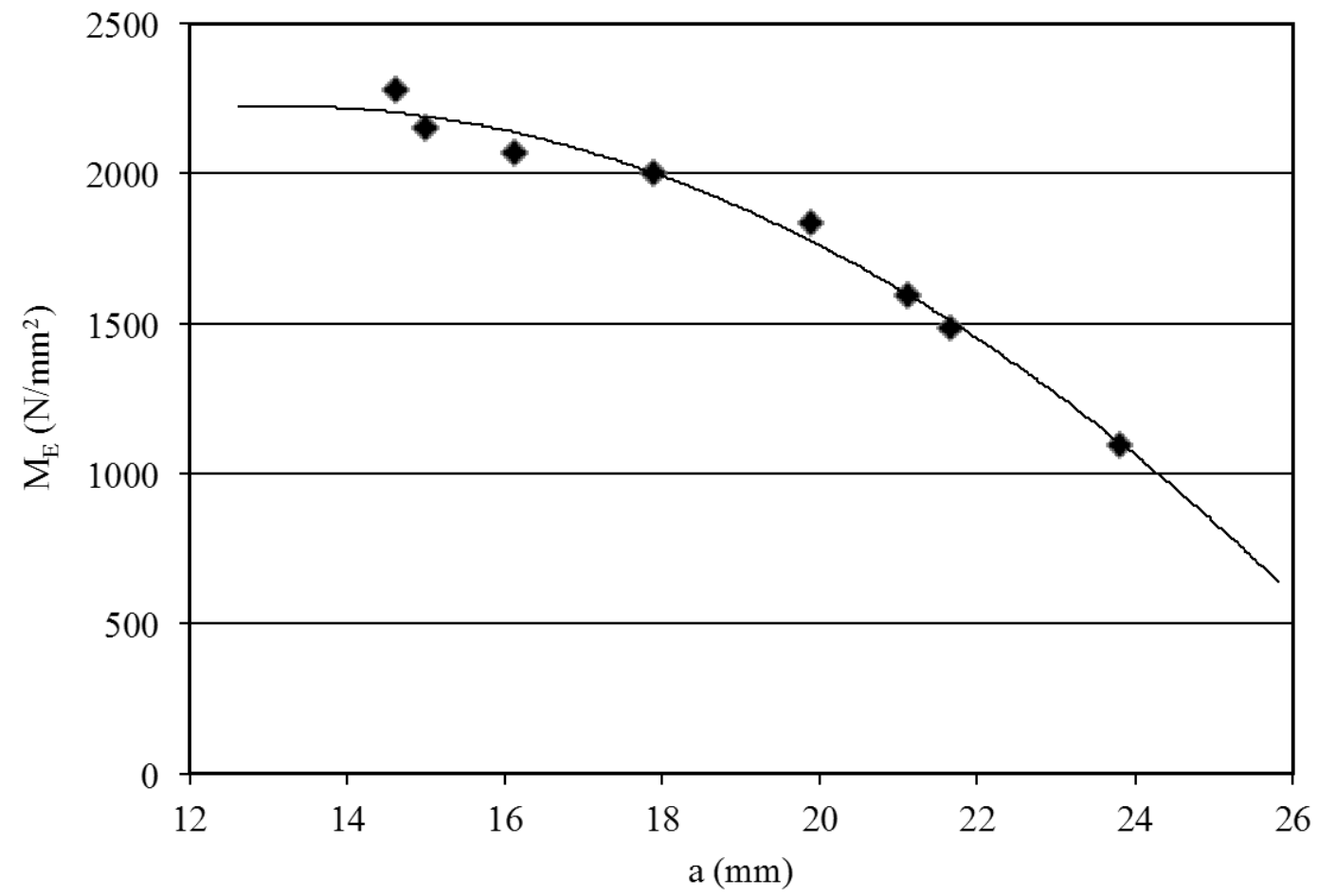

Figure 44: Modulus vs. crack length calibration of the sample configuration used in this work.

The values of $F$ and $d$ for the samples were recorded throughout the duration of the SCC-CGR experiments using data acquisition software. These values were used to calculate the modulus of each sample. The values of modulus were correlated to a crack length using the $M_{E}$ vs. $a$ calibration (Figure 44). The crack length and load values were used to calculate stress intensity using a strain energy release rate technique [72]. The details of the strain energy release rate calibration are given in Appendix A. A schematic of the experimental setup used to determine crack growth rates and stress intensities is illustrated in Figure 45 


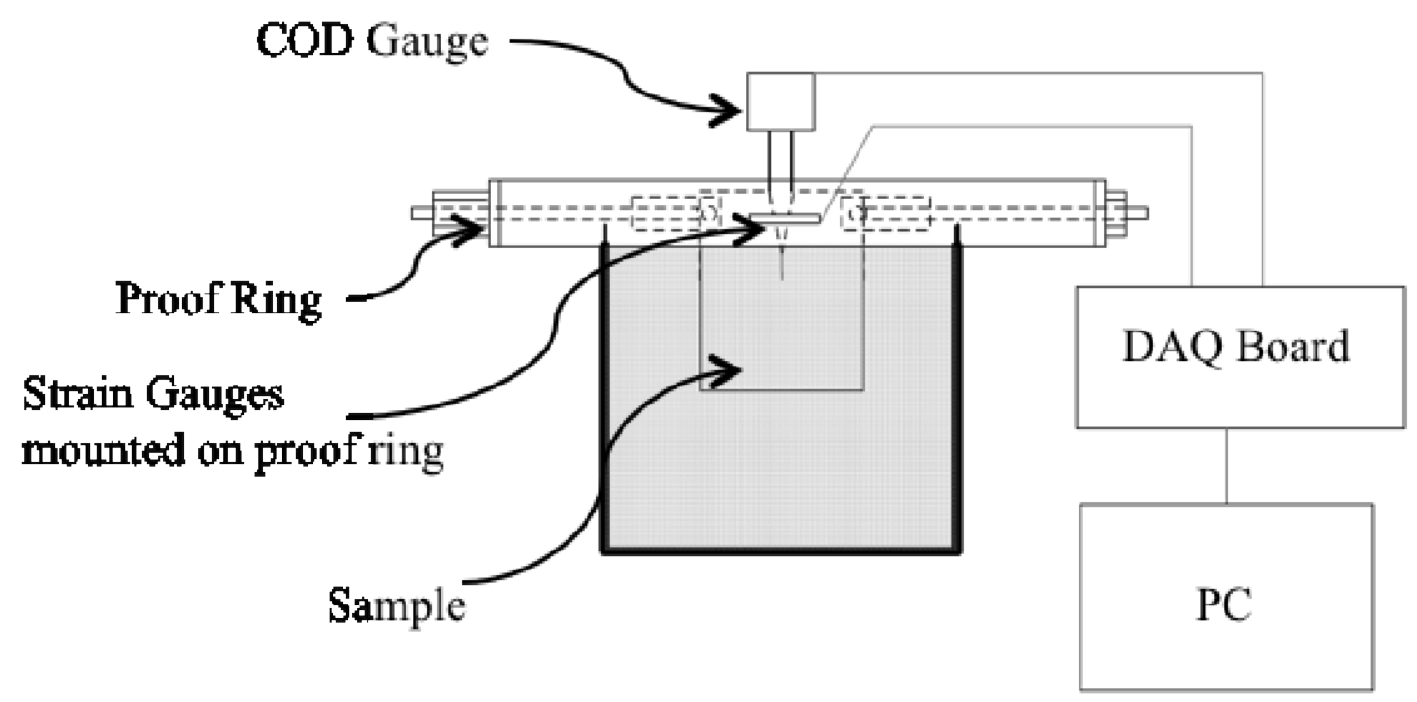

Figure 45: Schematic of setup that was used for SCC-CGR experiments. A static load was applied to a compact tensile sample using a proof ring. The load was monitored using strain gauges attached to the ring, and the crack opening displacement was monitored using a COD gauge. The signal was read by a data acquisition board, and complied on a PC. The recorded data was used to determine the change in modulus over time. The modulus is converted to a crack length using the strain energy release rate calibration technique.

The setup for conducting SCC-CGR studies was tested by performing a SCCCGR experiment on a compact tensile specimen of naturally aged AA7475 sheet exposed to $3.5 \% \mathrm{NaCl}$. The SCC behavior of wrought AA7475 is well documented, therefore making it a good model material on which to test the validity of the experimental technique described above. Results from a CGR experiment of naturally aged AA7475 are illustrated in Figure 46. The values of $K_{I S C C}$ and the stage II crack velocity were observed to be $\sim 17.9 \mathrm{MPa} \sqrt{\mathrm{m}}$ and $\sim 2 \times 10^{-4} \mathrm{~mm} / \mathrm{s}$, respectively. The observed value of $K_{I S C C}$ corresponds reasonably well to findings of Onoro who found that the value of $K_{I S C C}$ ranged from $\sim 8 M P a \sqrt{m}$ to $\sim 22 M P a \sqrt{m}$ in an alloy similar 
to AA7475 exposed to $3.5 \% \mathrm{NaCl}$ [65]. The observed value of stage II crack velocity is also similar to that observed by Onoro and Bovard who found the stage II crack velocity in similar alloys to range from $\sim 10^{-4} \mathrm{~mm} / \mathrm{s}$ to $\sim 10^{-3} \mathrm{~mm} / \mathrm{s}$ in alloys similar to AA7475 $[73,74]$ It was therefore concluded that the experimental setup permitted accurate measurement of SCC behavior in AA6005A extrusions.

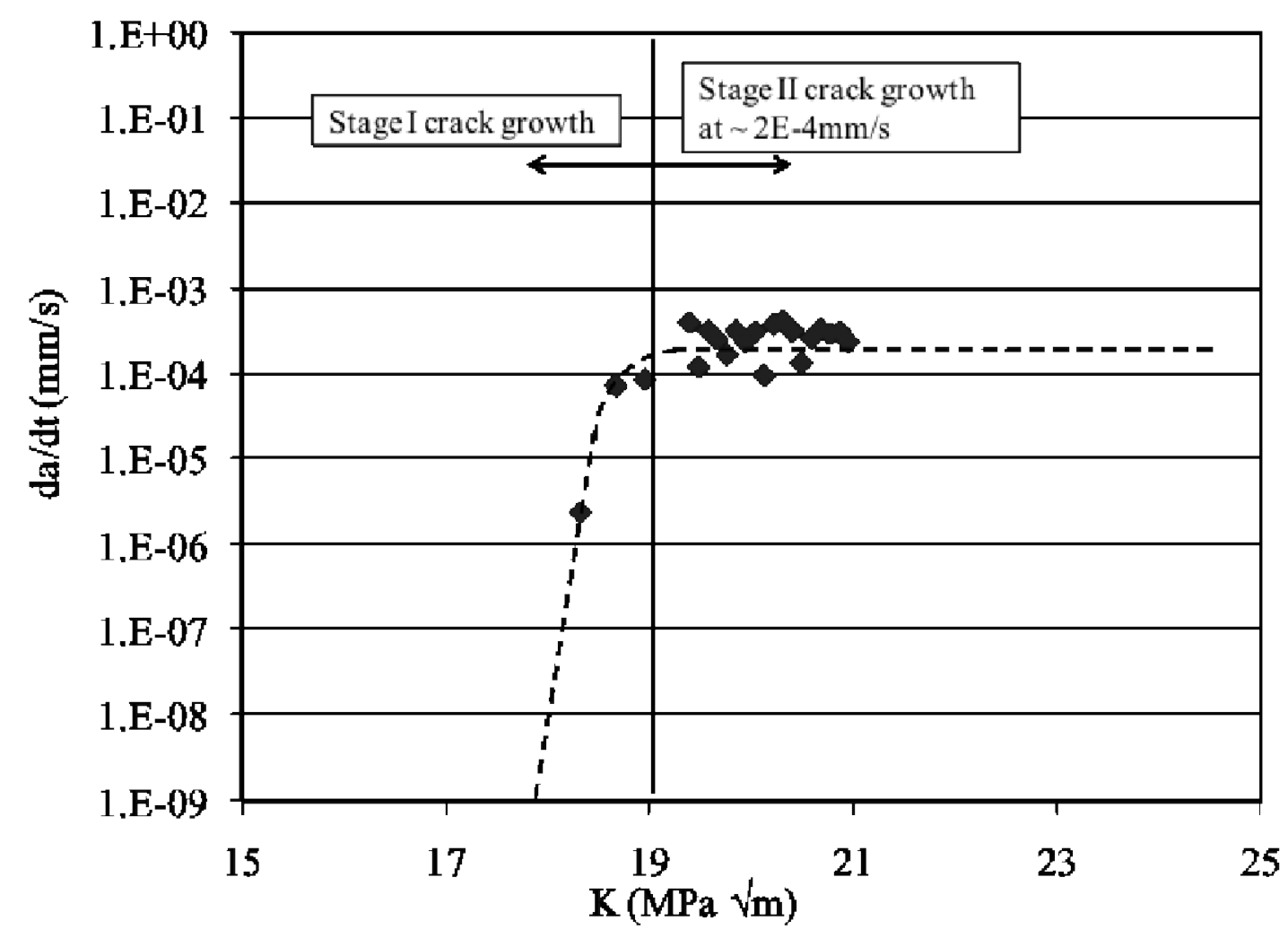

Figure 46: Results from CGR experiment of AA7475 sheet exposed to aerated 3.5\% $\mathrm{NaCl}$. The values of $K_{I S C C}$ and stage II crack growth rate agree well with published literature. These results suggest that the experimental setup used for the quantitative measurement of SCC in AA6005A extrusion is valid. 
Upon completing SCC-CGR experiments the specimens were fractographically characterized using optical microscopy and scanning electron microscopy (SEM). Optical microscopy was conducted to study the morphology of the cracks. Specific features investigated by optical microscopy included the crack branching, and the shape of the crack front after SCC. SEM was conducted to characterize the mode (brittle vs. ductile, intergranular vs. transgranular, etc.) of cracking along the fracture surface.

\subsubsection{Results - Quantitative SCC of AA6005A}

The stress corrosion crack growth rate (CGR) along direction 1 in AA6005AT6 extrusions was found to be nearly constant at $\sim 3 \times 10^{-5} \mathrm{~mm} / \mathrm{s}$ over a wide range of stress intensities when tested in an aerated solution containing $3.5 \% \mathrm{NaCl}$ at $\mathrm{pH}=$ 1.50 (Figure 47). When $K_{I}<8.5 M P a \sqrt{m}$, the observed crack velocity was approximately $3 \times 10^{-6} \mathrm{~mm} / \mathrm{s}$, and below this stress intensity the crack growth rate became too small to accurately measure.

Though it appears that the $d a / d t$ vs. $K$ behavior of AA6005A-T6 may correspond to typical SCC behavior (Figure 7) it should be noted that such a large range of Stage II crack growth is not typically observed in aluminum alloys. It is also noteworthy that the observed crack velocity of $\sim 3 \times 10^{-5} \mathrm{~mm} / \mathrm{s}$ at $10 \mathrm{MPa} \sqrt{\mathrm{m}}<K_{I}<$ $28 \mathrm{MPa} \sqrt{\mathrm{m}}$ is slow relative to many aluminum alloys [30, 31, 51, 55, 61-63, 74]. For comparison, typical stage II crack growth rates in 7xxx alloys exposed to neutral 
saltwater solutions with cracks growing along the extrusion direction are approximately $10^{-5}-10^{-3} \mathrm{~mm} / \mathrm{s}$.

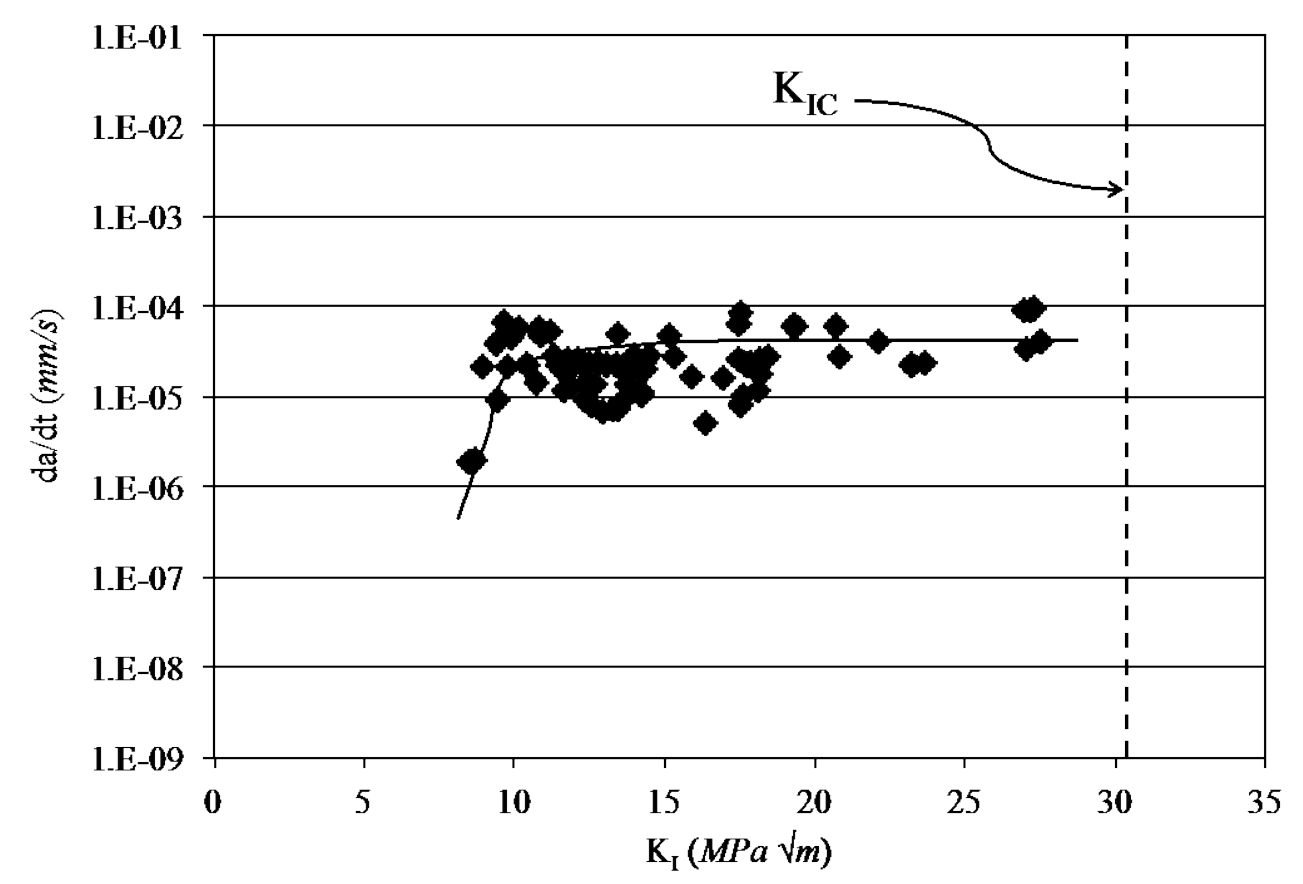

Figure 47: Crack growth rate of AA6005A-T6 extrusions exposed to aerated 3.5\% $\mathrm{NaCl}$ at $\mathrm{pH}=1.50$. The crack growth rate was relatively constant at $\sim 3$ $\times 10^{-5} \mathrm{~mm} / \mathrm{s}$ when $10 \mathrm{MPa} \sqrt{\mathrm{m}}<K_{I}<28 \mathrm{MPa} \sqrt{\mathrm{m}}$.

Fractography revealed evidence crack tunneling on the fracture surfaces.

Figure 48 illustrates a typical fracture surface observed on AA6005A-T6 after SCCCGR testing. The crack front advances most rapidly near the core of the material and least rapidly along the surface of the material during SCC testing. The significance of this observed crack tunneling is discussed in section 4.5.4. 


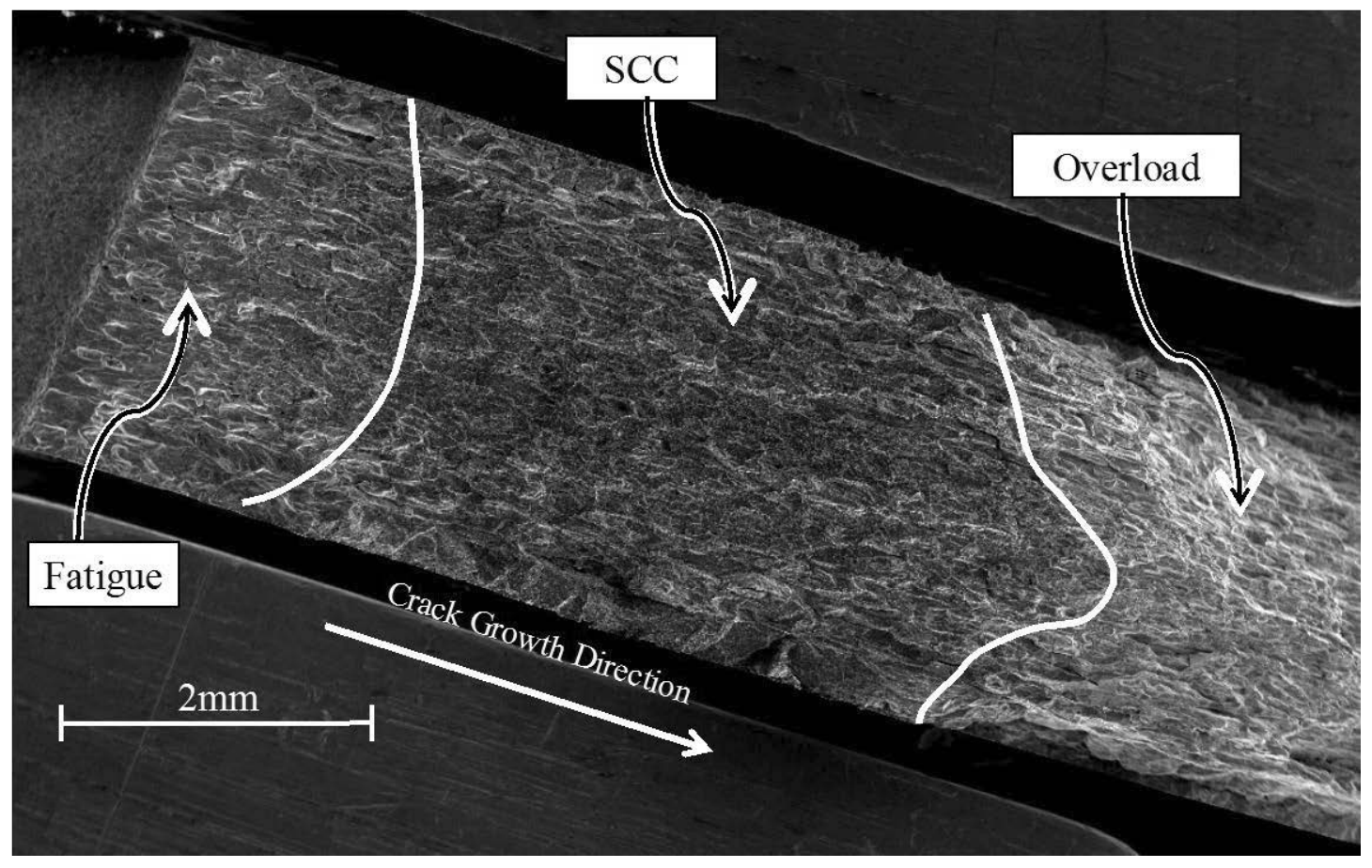

Figure 48: Typical SEM micgrograph of AA6005A-T6 fracture surface after conducting SCC testing in $3.5 \% \mathrm{NaCl}$ at $\mathrm{pH}=1.50$ for $40 \mathrm{hrs}$. Evidence of crack tunneling was observed.

Fractures surfaces of AA6005A-T6 CT specimens showed evidence of both brittle intergranular fracture and ductile fracture in regions of SCC (Figure 49). The mix of ductile and brittle fracture along the fracture surface suggests that SCC is occurring along small "channels" in the material, and the remainder of the material between these channels is failing by overload. The mix of brittle and ductile fracture along the fracture surface suggests that SCC growth is discontinuous along the crack front. In this case, SCC would occur only along discrete corrosion paths (regions of intergranular fracture), and regions between the intergranular SCC are fractured in a ductile manner when the fracture criterion is satisfied for the remaining ligament. 
Further analysis of the local stress state near the crack tips is discussed in section

\subsection{4.}

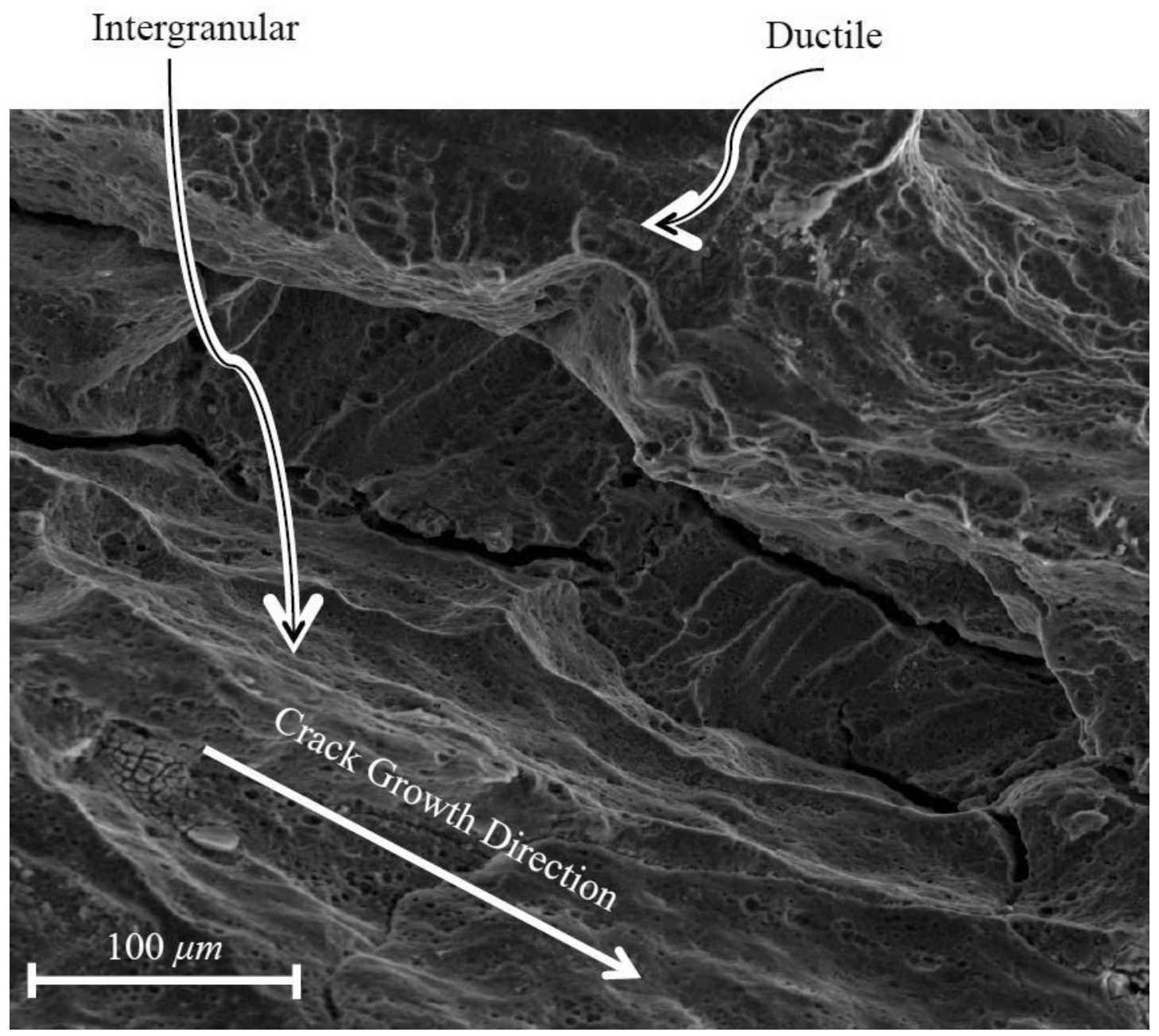

Figure 49: SEM micrograph of fracture surface within elongated grain structure of AA6005A-T6 CGR sample exposed to a solution of $3.5 \% \mathrm{NaCl}$ at $\mathrm{pH}=1.5$. Micrograph was taken near the tip of the stress corrosion crack at the midplane of the sample. Evidence of both brittle intergranular fracture and ductile fracture were observed in regions of SCC.

The morphology of the growing cracks in AA6005A-T6 was further examined by conducting optical microscopy along the 2 direction near the mid-plane of the material (Figure 50). A small spacer was placed near the end of the triangular wire 
EDM notch prior to unloading the CT samples used in this analysis. The purpose of the spacer was to retain the shape of the crack when the crack growth terminated without applying any additional stress that may distort the sample. The prepared samples were etched using Keller's reagent $\left(2 \mathrm{~mL} \mathrm{HF}+3 \mathrm{~mL} \mathrm{HCl}+5 \mathrm{~mL} \mathrm{HNO} \mathrm{H}_{3}+\right.$ $198 \mathrm{~mL} \mathrm{H}_{2} \mathrm{O}$ ) to reveal the grain structure.

The cracks appeared to advance exclusively along grain boundaries when examined using optical microscopy (Figure 50). The opposing fracture surfaces also appear to mate relatively well, indicating that very little bulk corrosion was occurring within the fracture surface. There was some evidence of crack branching near the crack tip. Crack branching is a feature commonly observed in SCC of $A l$ alloys, though it is often much more extensive than observed here [19]. Furthermore, some evidence of crack blunting was observed behind the crack tip. The mix of brittle and ductile fracture observed along the fracture surface (Figure 49) suggests the crack blunting is likely due to discontinuous crack growth that occurs after local plastic deformation near the crack tip. 


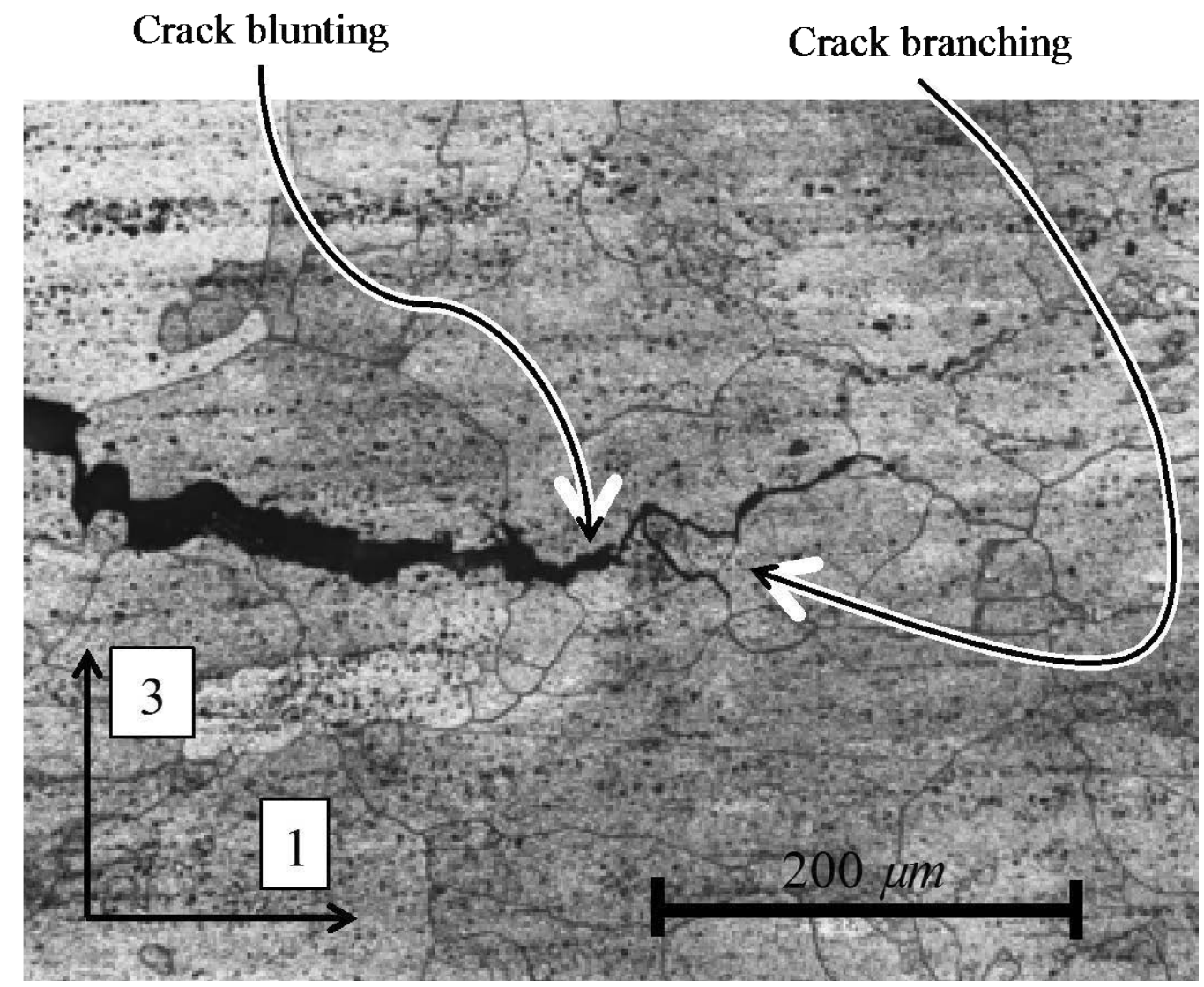

Figure 50: Morphology of advancing crack in AA6005A-T6 exposed to 3.5\% $\mathrm{NaCl}$ at $p H=1.5$. The total stress corrosion crack length in this sample was 4.24 mm. The cracks appeared to advance along the grain boundaries. There was evidence of crack blunting away from the crack tip, and crack branching near the crack tip.

Addition of $0.3 \% \mathrm{As}_{2} \mathrm{O}_{3}$ to the test solution did not have a significant influence on the observed crack growth rate of the alloy (Figure 51). The average crack velocity in CT specimens tested in a solution containing $0.3 \% \mathrm{As}_{2} \mathrm{O}_{3}$ was $\sim 3.3$ $\times 10^{-5} \mathrm{~mm} / \mathrm{s}$, whereas the average crack velocity in CT specimens tested in solutions without $0.3 \% \mathrm{As}_{2} \mathrm{O}_{3}$ was $\sim 3.0 \times 10^{-5} \mathrm{~mm} / \mathrm{s}$. These results are not consistent with what would be expected if SCC in AA6005A-T6 extrusions were primarily a hydrogen 
embrittlement phenomenon, as there was no enhancement in CGR with the addition of an HRP to the test solution.

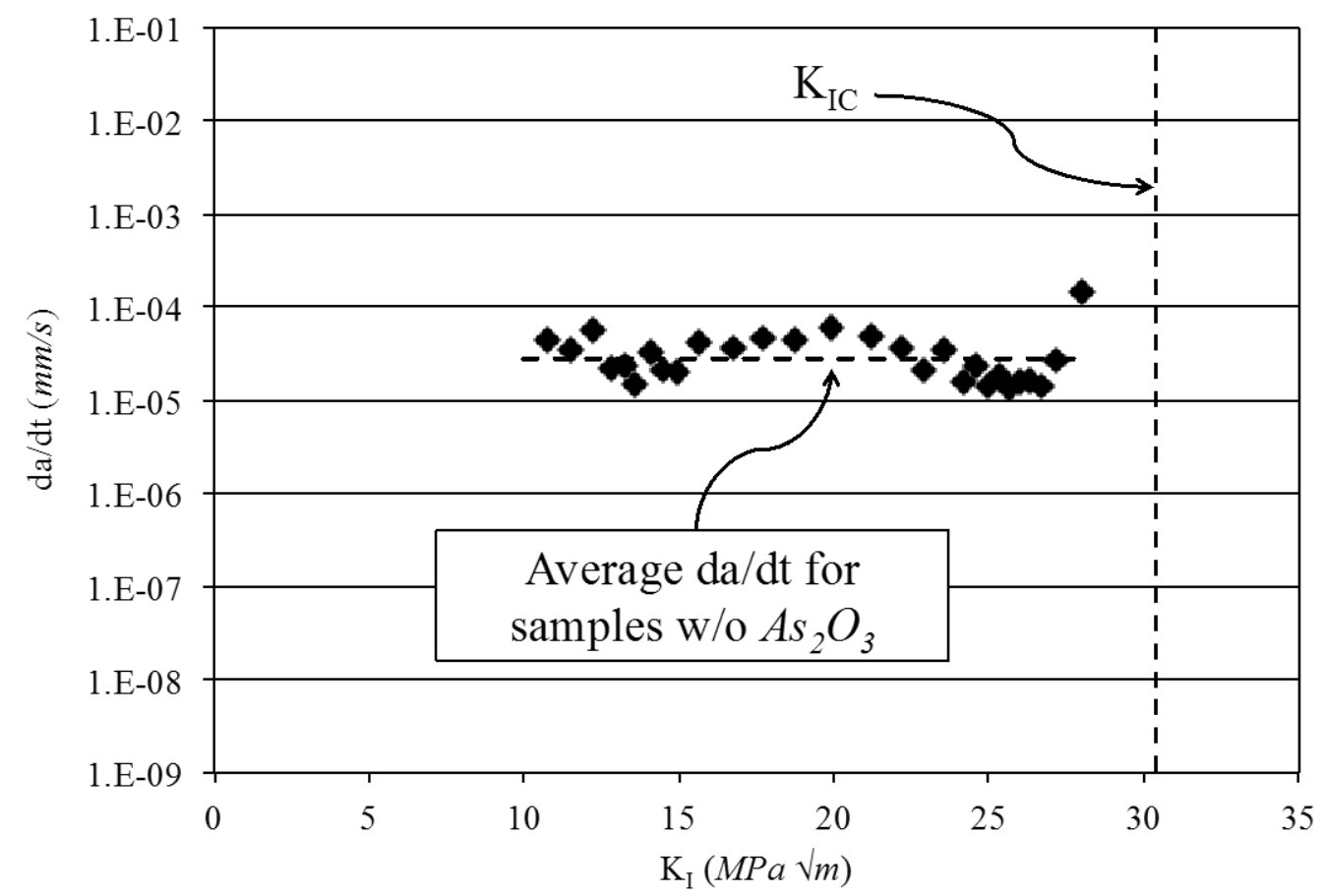

Figure 51: Effect of a hydrogen recombination poison $\left(\mathrm{As}_{2} \mathrm{O}_{3}\right)$ on the crack growth rate of $\mathrm{AA6005A-T6.} \mathrm{The} \mathrm{addition} \mathrm{of} \mathrm{As}_{2} \mathrm{O}_{3}$ did not enhance the observed crack growth rate, suggesting that hydrogen embrittlement is not an operating mechanism of SCC in AA6005A-T6.

Fractography of SCC-CGR specimens exposed to solutions containing $\mathrm{As}_{2} \mathrm{O}_{3}$ revealed mechanisms of crack growth that are similar to those of specimens exposed to solutions without $\mathrm{As}_{2} \mathrm{O}_{3}$. A mix of brittle and ductile fracture was observed along the fracture surface in samples tested in the solutions containing $\mathrm{As}_{2} \mathrm{O}_{3}$ (Figure 52). These results indicate that the addition of a hydrogen recombination poison to solution does not significantly alter the mechanisms of crack growth in AA6005A-T6, 
and that the mechanism of SCC in AA6005A-T6 exposed to acidified saltwater is not influenced by changes in the hydrogen discharge reaction. This, in turn, suggests that the SCC mechanism in this alloy could operate primarily by anodic dissolution. 


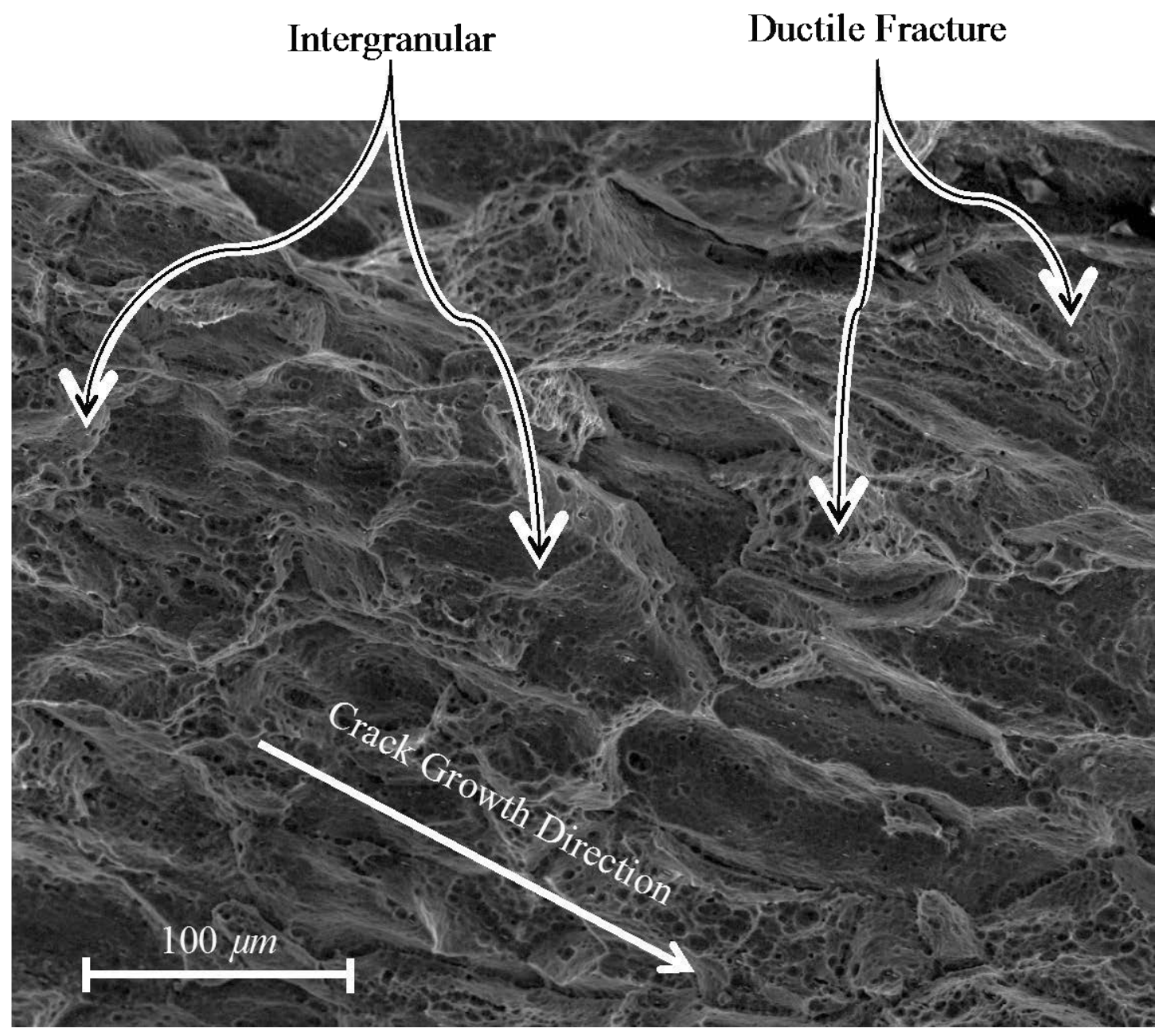

Figure 52: Fracture surface of AA6005A-T6 CGR specimen exposed to a solution of $3.5 \% \mathrm{NaCl}+0.3 \% \mathrm{As}_{2} \mathrm{O}_{3}$ at $\mathrm{pH}=1.5$. Micrograph was taken near the tip of the stress corrosion crack at the midplane of the sample. Evidence of brittle and ductile fracture along the fracture surface indicates that the mechanism of crack growth is similar to that of a sample exposed to a solution without $\mathrm{As}_{2} \mathrm{O}_{3}$.

\subsubsection{Discussion of Quantitative SCC Analysis}

Recall that diffusion of $\mathrm{Al}^{3+}$ away from the tip of an IGC path was found to be the primary factor limiting the velocity of IGC in the case of unstressed samples (Section 4.3.3). The possibility that the velocity of SCC in AA6005A-T6 is also 
limited by diffusion of $A l^{3+}$ away from the crack tip was examined by applying the diffusion-based model discussed in section 4.3.3. Figure 53 illustrates the experimentally observed relationship between the structure corrected corrosion velocity and the structure corrected corrosion path length for both loaded CT samples (SCC-CGR) and unstressed (IGC) samples. The structure corrected corrosion velocity (vertical axis) and corrosion path length (horizontal axis) were deduced using Equation 6. The structure-corrected corrosion path length in the dataset for the loaded CT samples $(\diamond)$ corresponds to the corrosion path length only in regions of SCC (does not include fatigue precrack). The structure corrected corrosion path length in the dataset for unstressed samples $(\diamond)$ corresponds to the total calculated path length between the external environment and the tip of an IGC path along direction 1.

Figure 53 demonstrates that there is minimal difference in corrosion velocity between the stressed and unstressed samples at small corrosion path lengths $(<0.25$ $\mathrm{mm}$ ). As the corrosion path length increases beyond about $0.25 \mathrm{~mm}$, the structure corrected IGC crack velocity for the unstressed samples decreases, while the SCC crack velocity for the loaded CT samples remains approximately constant at $\sim 3 \times 10^{-5}$ $\mathrm{mm} / \mathrm{s}$.

Since the observed SCC velocity was independent of crack length (Figure 53), the effective diffusion path length for $A l^{3+}$ away from the crack tip must be constant if diffusion of $\mathrm{Al}^{3+}$ is a limiting factor to crack growth in loaded CT samples of AA6005A-T6 according to Equation 14. It is unreasonable to assume that the shortest diffusion path length for $A l^{3+}$ would be along the direction $\mathbf{1}$, as the lengths of all 
cracks (including SCC and fatigue precrack) in the SCC-CGR experiments were larger than the specimen thickness. The length of the cracks along direction $\mathbf{1}$ are also continuously increasing during SCC-CGR experiments, thereby decreasing the driving force for $A l^{3+}$ diffusion along $\mathbf{1}$ as the cracks become longer (which is inconsistent with observed $v$ vs. $L$ behavior, Figure 53). Therefore if diffusion of $A l^{3+}$ away from the crack tip were the limiting factor in determining the velocity of SCC in the case of loaded CT samples, it can be assumed that $A l^{3+}$ must migrate along the 2 direction.

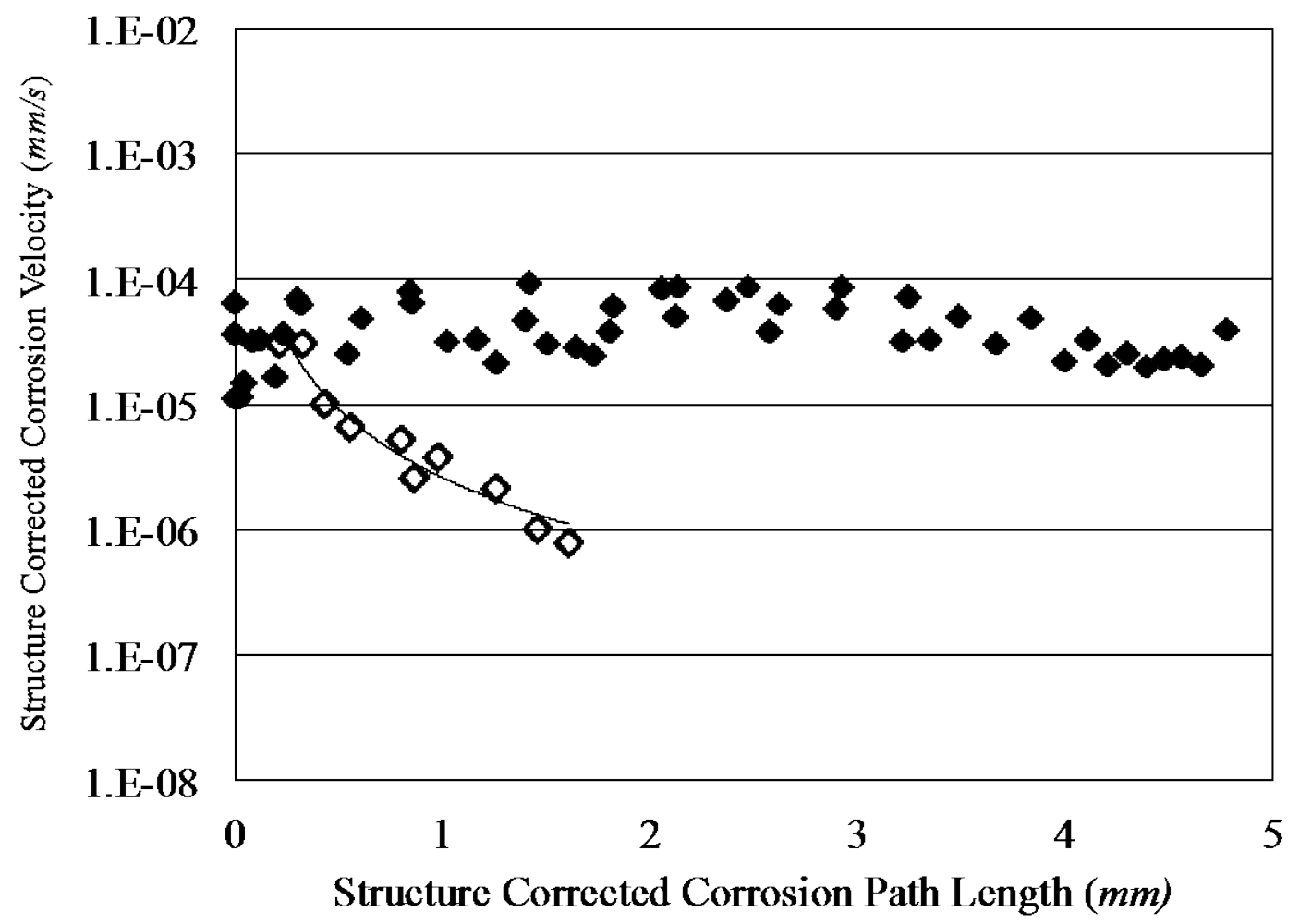

Figure 53: Structure-corrected corrosion velocity with increasing corrosion depth along direction 1 for stressed ( ) and unstressed $(\Delta)$ samples. A significant decrease in velocity was observed with increasing corrosion depth in the absence of an applied stress. 
The effective corrosion (and diffusion) path length, $L$, along the $\mathbf{2}$ direction would be approximately equal to the distance between a point on the crack front and the external environment (Figure 54). The crack front was assumed to be a straight line oriented perpendicular to the crack growth direction. This crack front geometry used does not accurately model the crack tunneling observed in the SCC-CGR samples, but should allow reasonable estimation of the diffusion path length for $\mathrm{Al}^{3+}$ along direction 2. In this case, SCC velocity should not depend on crack length since the effective diffusion path length would not vary with crack length. 


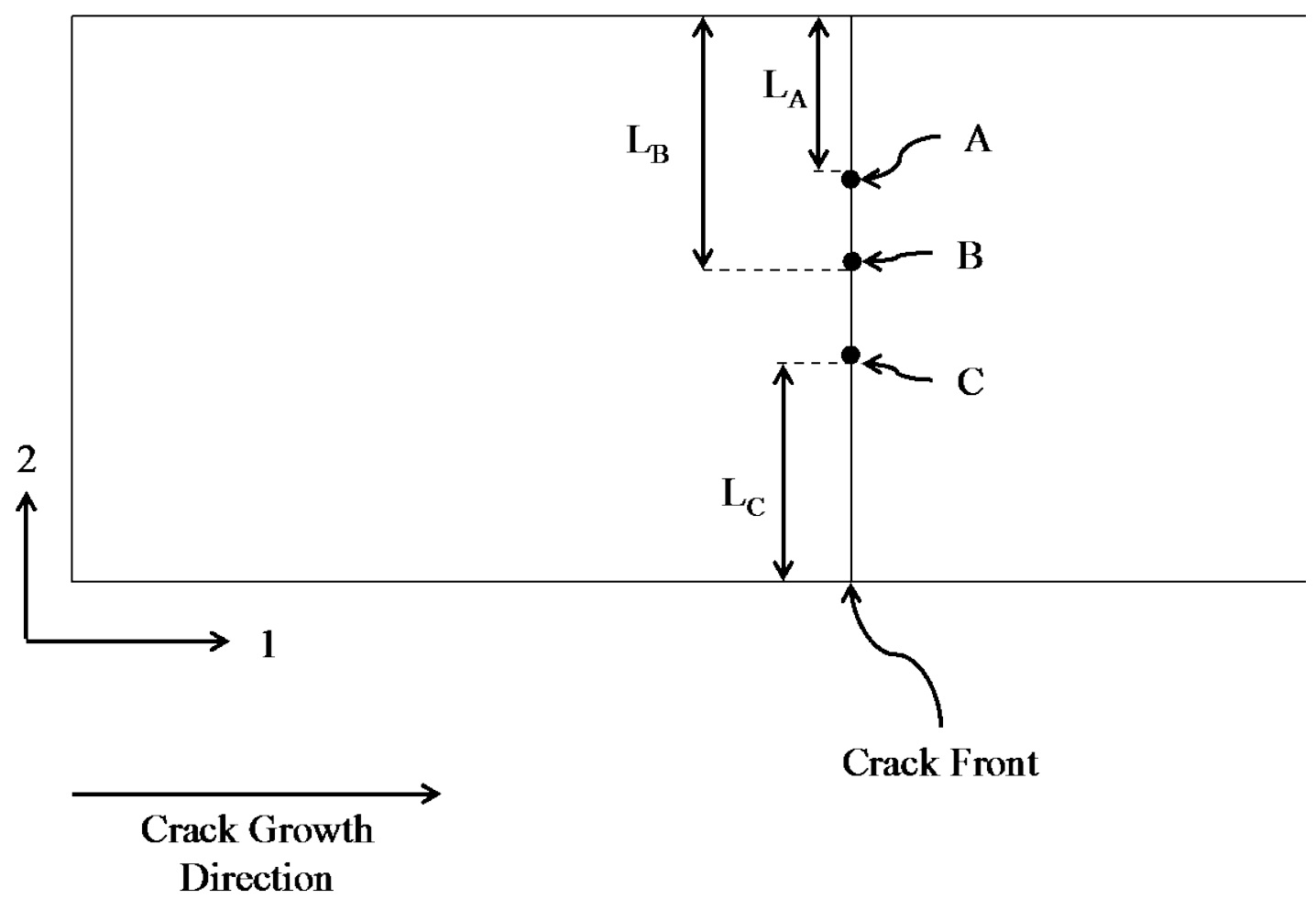

Figure 54: Effective corrosion (and diffusion) path lengths along direction 2 for stressed SCC-CGR specimens with crack lengths greater than the 1/2 thickness of the sample. When the crack length exceeds the $1 / 2$ thickness of the sample, the shortest corrosion path length between the crack front and the external environment would correspond to the distance between that point and the external environment along the 2 direction. The effective corrosion path length for point $A$ in this figure is $L_{A}$. Similarly, the corrosion path lengths for points $B$ and $C$ are $L_{B}$ and $L_{C}$, respectively.

Equation 14 was applied to calculate the crack front velocity at the centerline of a CT sample, and to generate a profile of SCC velocity along the crack front. These calculations were made assuming $A l^{3+}$ diffuses exclusively along direction $\mathbf{2}$, and the observed $p H$ vs. diffusion path length behavior (Figure 34) is valid for both unstressed and stressed samples. With these assumptions, the velocity at the centerline of a CT specimen was calculated to $3.89 \times 10^{-7} \mathrm{~mm} / \mathrm{s}$, which is $\sim 100 \mathrm{X}$ 
smaller than the observed constant SCC velocity $\left(\sim 3 \times 10^{-5} \mathrm{~mm} / \mathrm{s}\right)$. This result suggests that diffusion of $A l^{3+}$ away from the crack tip is not the primary limiting factor in determining the velocity of SCC in AA6005A-T6. Furthermore, if diffusion of $A l^{3+}$ away from the crack tips along direction $\mathbf{2}$ were the limiting factor in determining SCC velocity of AA6005A-T6, the crack velocity would be least along the centerline of the material and greatest along the edges of the material, as shown in Figure 55. The predicted velocity profile at the crack front is not consistent with the observed crack tunneling during SCC-CGR experiments (Figure 48). Therefore, in contrast to IGC in unstressed samples of AA6005A-T6, it does not appear that the SC crack velocity in the case of loaded CT samples is limited by diffusion of $A l^{3+}$ along the 2 direction. 


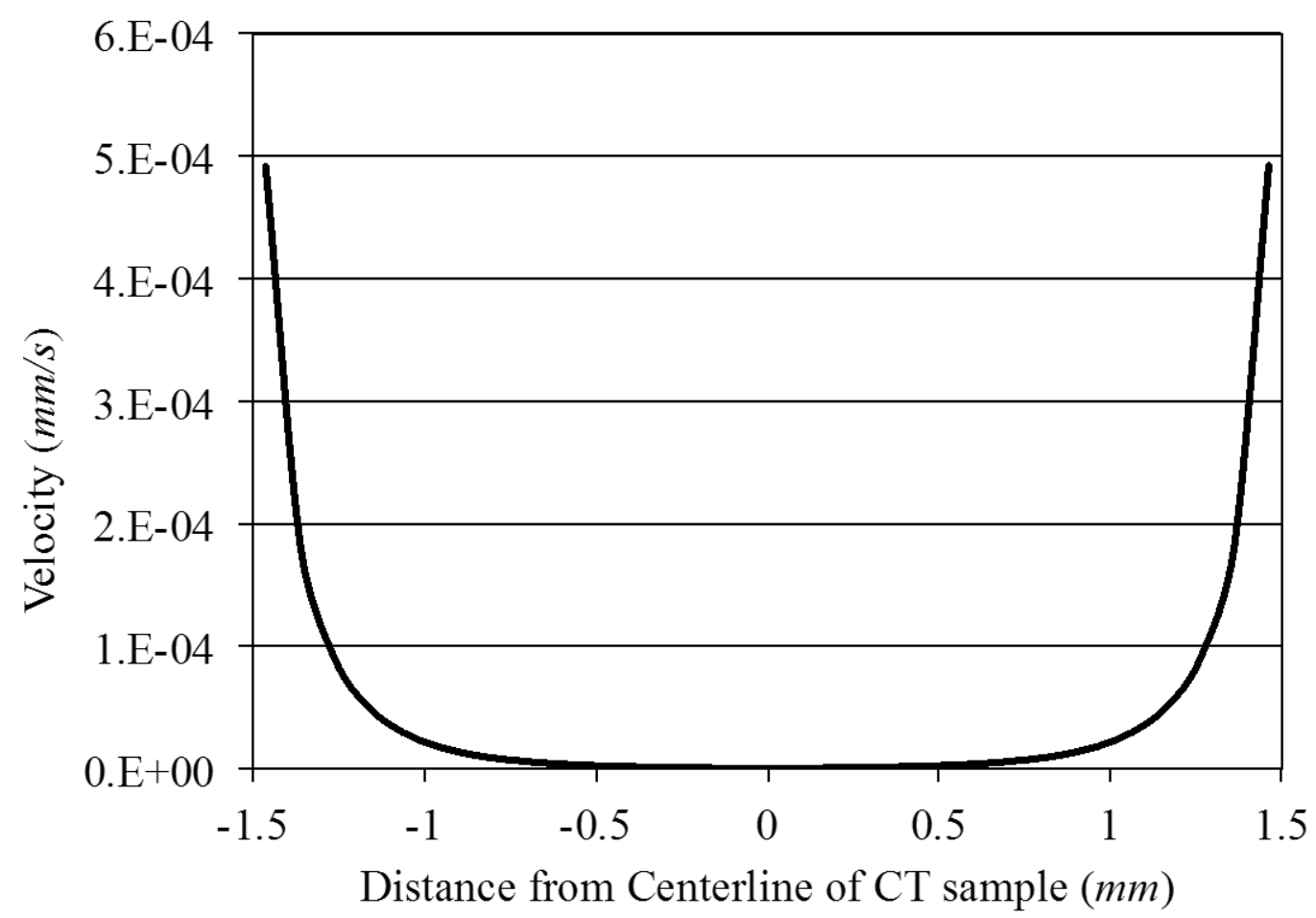

Figure 55: Calculated velocity profile along the crack front of a CT sample of AA6005A-T6 exposed to $3.5 \% \mathrm{NaCl}$ at $\mathrm{pH}=1.5$. The velocities at various points along the crack front were calculated by applying Equation 14 .

Recall that the addition of a hydrogen recombination poison to the test solution did not have a significant influence the SCC susceptibility of AA6005A-T6. This result suggests that hydrogen embrittlement is not the dominant mechanism of SCC in AA6005A-T6. Additional analysis of the local stress state and electrochemical reactions at an SC crack tip was performed to further investigate the possibility that anodic dissolution may be the primary mechanism of SCC in AA6005A-T6. 


\subsubsection{Effects of Stress Triaxiality and Local Electrochemical Reactions on Stress Corrosion Cracking of AA6005A-T6}

Crack tunneling was observed along the fractures surfaces of loaded samples exposed to $3.5 \% \mathrm{NaCl}$ at $\mathrm{pH}=1.5$ (Figure 48). In linear elastic fracture mechanics (LEFM), the phenomenon of crack tunneling is generally attributed to a local in maximum stress triaxiality just ahead of the crack tip near the center plane of the material. Factors leading to triaxiality of the stress state at the specimen mid-plane near the crack tip are illustrated schematically in Figure 56. Because normal tractions are absent on the 1-3 surface, that surface is always in a state of plane stress $\left(\sigma_{2}=0\right)$. When the plastic zone near the crack tip is large (Figure 56B), through-thickness thinning of the specimen minimizes $\sigma_{2}$ at the specimen mid-plane thereby minimizing triaxiality of the stress state. When the plastic zone is small (Figure 56A), throughthickness elastic constraint around the crack tip leads to values of $\sigma_{2}$ that approach the value of $\sigma_{3}$. Similar reasoning leads to the conclusion that elastic constraint gives values of $\sigma_{1}$ that are approximately equal to $\sigma_{3}$. When $\sigma_{1}=\sigma_{2}=\sigma_{3}$, a state of perfect stress triaxiality is achieved $[72,75]$. The significance of perfect triaxiality is that while the principle stresses $\left(\sigma_{1}, \sigma_{2}\right.$, and $\left.\sigma_{3}\right)$ may be significant, the shear stresses will vanish. Low shear stresses minimize plastic deformation, and the tensile stresses promote brittle facture over plastic deformation of the material. 

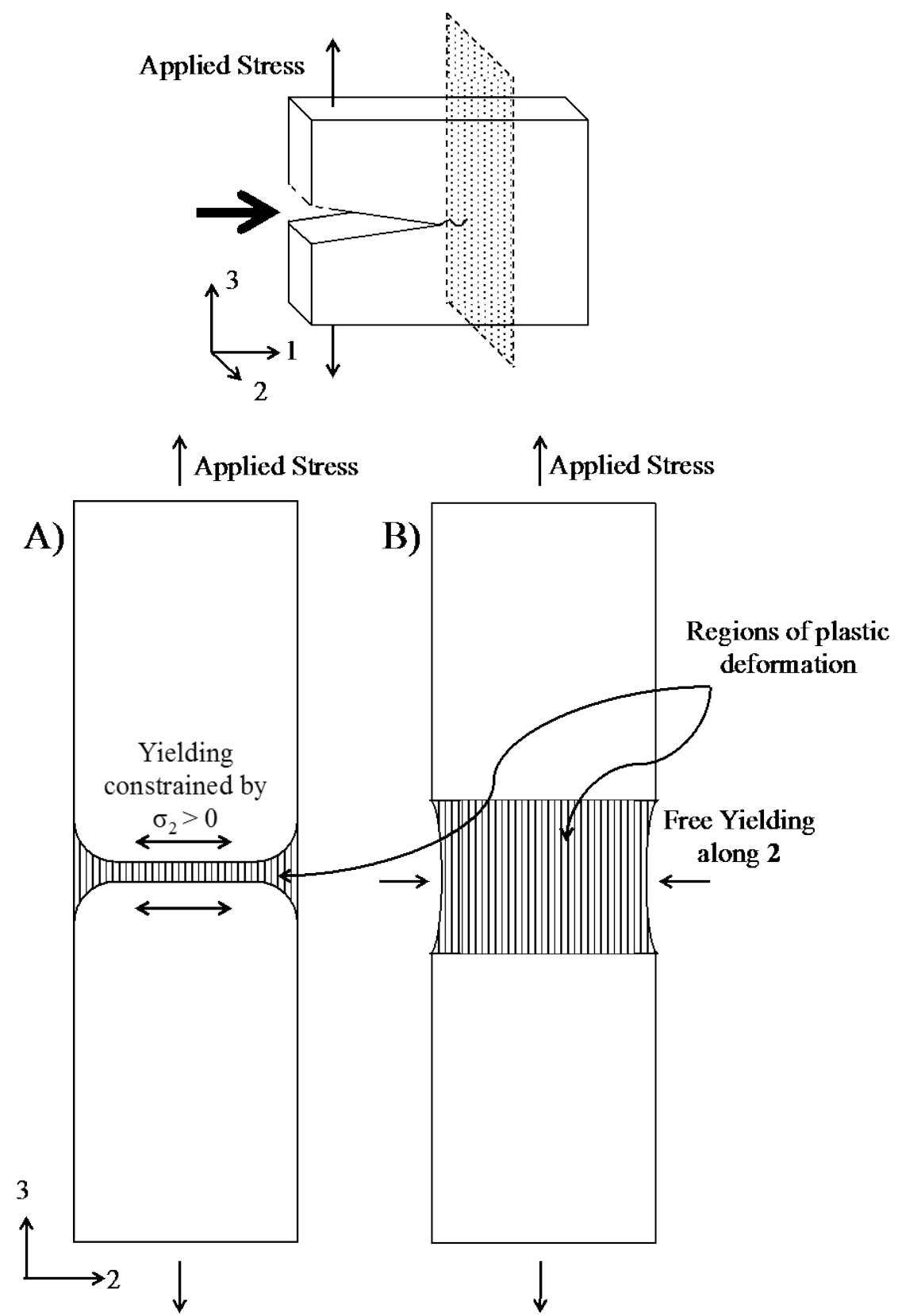

Figure 56: Effect of plastic zone size on the stress state experienced in a material. Figures $A$ and $B$ are looking at a plane ahead of the crack front along direction 1, as indicated by the dark arrow in the perspective view at the top. A) Surface deformation is limited by a large constraining stress oriented perpendicular to the loading axis when the plastic zone is small. B) Lateral deformation is observed if the plastic zone is sufficiently large. 
Figure 57 shows the morphology of a crack growing along the direction $\mathbf{1}$ in a compact tensile sample of AA6005A-T6 after SCC-CGR testing in a solution of $3.5 \%$ $\mathrm{NaCl}$ at $p H=1.5$. The specimen illustrated in Figures 57A-D was prepared by making serial sections of 3-2 planes at different depths along direction 1. Figure 57A shows the morphology of a growing SC crack in the 3-2 plane $1 \mathrm{~mm}$ beyond the end of the fatigue precrack. Similarly, Figures 57B-D show the morphology of a growing SC cracking the 3-2 plane 2, 3, and $4 \mathrm{~mm}$ beyond the end of the fatigue precrack. The total length of the SC crack for the sample shown in Figure 54 was approximately $4.08 \mathrm{~mm}$, so Figure 57D represents the morphology of the SC crack at a plane near the crack tip. Note that there is negligible plastic deformation along direction $\mathbf{2}$ at the surface of the specimen at all crack lengths. The lack of deformation along the free surfaces of the sample suggests that there is a region within the sample thickness that does not experience significant shear stresses, and would therefore be experiencing a significant degree of stress triaxiality. 

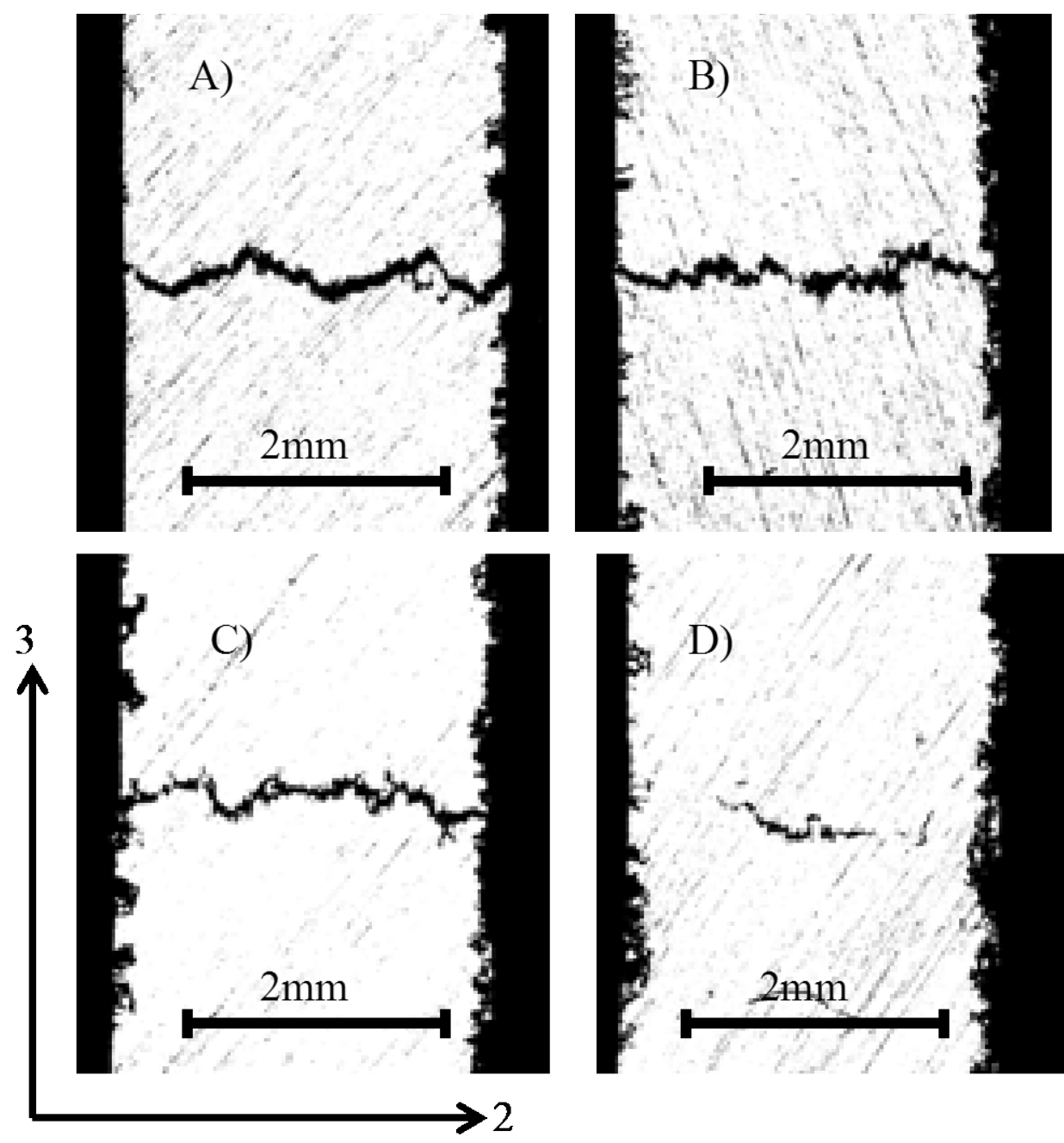

Figure 57: Crack morphology at various depths in a compact tensile bar of AA6005A-T6 exposed to $3.5 \% \mathrm{NaCl}$ at $\mathrm{pH}=1.5$. The crack is growing along direction 1. A) $1 \mathrm{~mm}$ beyond fatigue precrack. B) $2 \mathrm{~mm}$ beyond fatigue precrack. C) $3 \mathrm{~mm}$ beyond fatigue precrack. D) $4 \mathrm{~mm}$ beyond fatigue precrack. No evidence of deformation along the 2 direction was observed at any point beyond the fatigue Precrack.

Assume the crack tip plastic zone is sufficiently small to satisfy plane strain conditions near the center of the material, the maximum stresses experienced near the crack tip of AA6005A-T6 SCC-CGR specimens can be estimated using the plastic constraint factor (PCF). The PCF is defined as [75]: 


\section{Equation 16}

$$
P C F=\frac{\sigma_{\max }}{\sigma_{y}}
$$

Where $\sigma_{\max }$ is the maximum tensile stress near a crack tip, and $\sigma_{y}$ is the bulk yield stress of the material. In the case of plane strain loading, the value of $P C F$ approaches a value of 3 along the plane of the advancing crack [75]. Details of the calculations used to determine the PCF are given in Appendix B. The bulk yield stress of AA6005A-T6 is approximately $250 \mathrm{MPa}$, thus the maximum stress near the crack tip would be approximately $750 \mathrm{MPa}$. This probably overestimates the value of maximum stress at the crack tip, as the applied loads for the SCC-CGR experiments in this work correspond to a crack tip stress which is much lower than the bulk yield stress of the alloy according to the relationship $K_{I}=\sigma(a)^{1 / 2}$. However, this value gives a reasonable approximation of the stress state near the crack tip.

The effect of stress on the chemical potential near a crack tip can be estimated using the Nabarro-Herring $(\mathrm{NH})$ model $[76,77]$. The $\mathrm{NH}$ model is used to determine the change in free energy associated with a vacancy migrating from an unstressed (crack flank) to a stressed (crack tip) region (Figure 58). Though originally formulated to describe the driving force for high-temperature diffusional creep, the $\mathrm{NH}$ model has been applied to understand the driving force for dissolution in the case of SCC [78-82]. The NH model states:

\section{Equation 17}

$$
\Delta \mu=\frac{\sigma_{a p p}}{M V_{A l}}
$$


Where $\Delta \mu$ is the change in chemical potential associated with vacancy migration to a crack tip, $M V_{A l}$ is the molar volume of $A l$, and $\sigma_{a p p}$ is the local stress. If we assume the applied stress near the midplane of the $\mathrm{CT}$ specimens approaches $750 \mathrm{MPa}$, and take the molar volume of $\mathrm{Al}$ as $0.1 \mathrm{~mol} / \mathrm{cm}^{3}, \Delta \mu$ is found to be $7,500 \mathrm{~J} / \mathrm{mol}$.

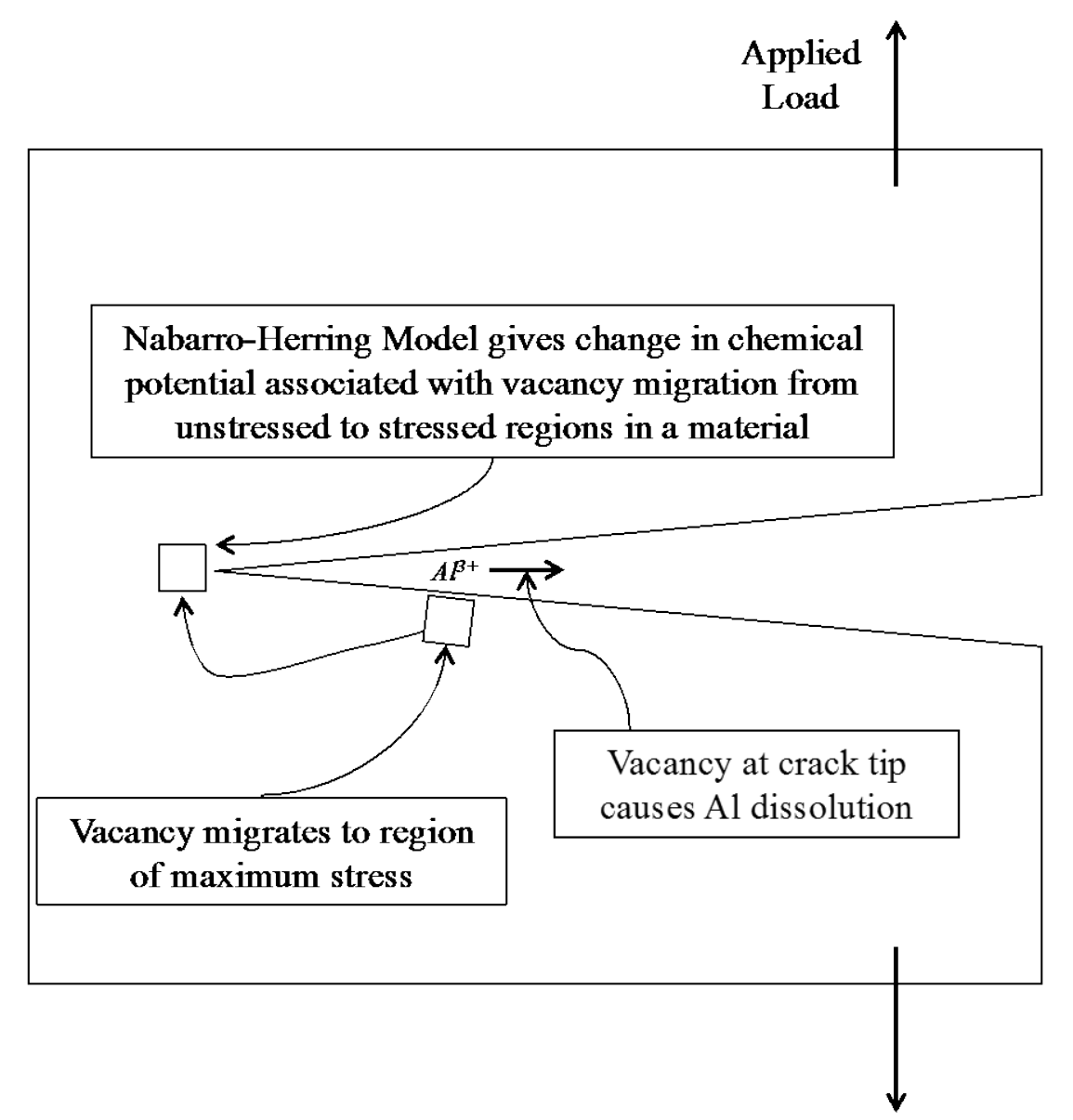

Figure 58: Schematic representation of the Nabarro-Herring model. Vacancies migrating from an unstressed region to a region of maximum stress results dissolution of the parent material and a decrease in chemical potential near the crack tip.

The change in electrochemical potential at the crack tip due to an applied load was related to the change in chemical potential using Faraday's law [83]: 


\section{Equation 18}

$$
\Delta V=\Delta \mu /{ }_{-n F}
$$

Where $\Delta V$ is the change in electrochemical potential, $n$ corresponds to the number of electrons involved in the dissolution reaction ( 3 for $A l$ ), and $F$ is Faraday's constant (96,500 C/mol). Applying equations $17-18$, the total change in electrochemical potential at the crack tip due to an applied load was estimated to be approximately $-0.026 V$. This change in electrochemical potential would shift the half-cell corrosion potential of the anodic reaction $\left(A l=A l^{3+}+3 e^{-}\right)$to a lower value, and should have no effect on the half-cell corrosion potential of the cathodic reaction.

Recall from section 4.3.3 that the $\mathrm{pH}$ at the tip of a corrosion path varied with corrosion path length. When the $p H$ of the bulk solution was held at 1.5 , the $p H$ at the tip of a corrosion path was found to reach an equilibrium value of $\sim 2.5$ as the corrosion path length approached $2.3 \mathrm{~mm}$. Assuming the effective corrosion path length is $1.58 \mathrm{~mm}$ (1/2 thickness of CT sample) for loaded CT samples of AA6005AT6, the $p H$ at the tip of the corrosion path is approximately 2.0 when the $p H$ of the bulk solution is 1.5 (Figure 34 ).

In the case of loaded CT samples of A6005A-T6 exposed to $3.5 \% \mathrm{NaCl}$ at $\mathrm{pH}$ $=1.5$, the surface area of stressed material exposed to a solution with $p H=2.0$ would be very small relative to the surface area of material exposed to solution with $p H$ $=1.50$ (Figure 59). Since electrons can move freely between the crack tip (where they are created by the anodic reaction) to the external surfaces of the sample (where they are consumed by the cathodic reaction), it is reasonable to assume that the anodic and 
cathodic reactions governing crack growth are occurring at different locations on the CT samples. With this assumption, the cathodic reaction on the unstressed surfaces would follow the polarization curve for AA6005A-T6 exposed to a solution with $p H$ $=1.50$, and the anodic reaction at the crack tip would follow the polarization curve for stressed AA6005A-T6 exposed to a solution with $p H=2.0$. It is also reasonable to assume that the overall corrosion reaction would be occurring at a potential near the corrosion potential for a sample exposed to a solution with bulk $p H=1.5$ since the majority of the sample is unstressed and exposed to the $p H=1.5$ solution. 


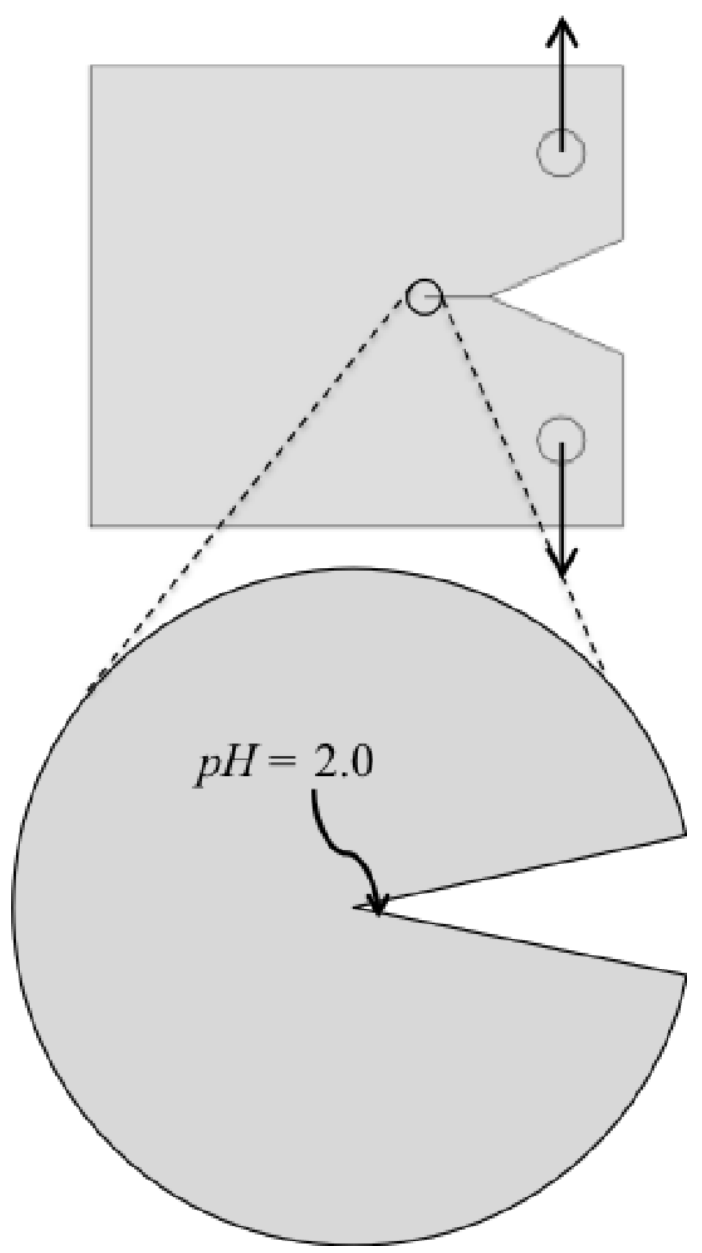

Figure 59: Only a small fraction of a loaded compact tensile bar will be exposed to solution at $\mathrm{pH}=2.0$ when the $\mathrm{pH}$ of the bulk solution is held at 1.50 .

First consider the electrochemical reactions occurring at a stress corrosion crack tip in a loaded CT sample of AA6005A-T6 that is exposed to a solution of $3.5 \%$ $\mathrm{NaCl}$ at $p H=1.5$ (Figure 60). In this case, the highly stressed crack tip will be exposed to a solution with $p H=2.0$, while the majority of the sample (which is in an unstressed state) will be exposed to a solution with $p H=1.5$. The anodic reaction occurring in the crack tip solution would be shifted downward by $\sim 0.026 V$ vs. SCE 
due to the local stress state at the crack tip, and the cathodic reaction would follow the polarization curve for an unloaded sample exposed to a $p H=1.5$ solution. Since most of the sample is exposed to a $p H=1.5$ solution, it is reasonable to assume that the anodic reaction at the crack tip will be occurring at the value of $E_{C o r r}$ when a sample exposed to the $p H=1.5$ solution $(-0.71 V v s . S C E)$. With these assumptions, the anodic corrosion current density at the crack tip in the case of a loaded CT sample exposed to a solution of $3.5 \% \mathrm{NaCl}$ at $\mathrm{pH}=1.5, i_{1.5}$, should be approximately $4.6 \times 10^{-4}$ $\mathrm{A} / \mathrm{cm}^{2}$.

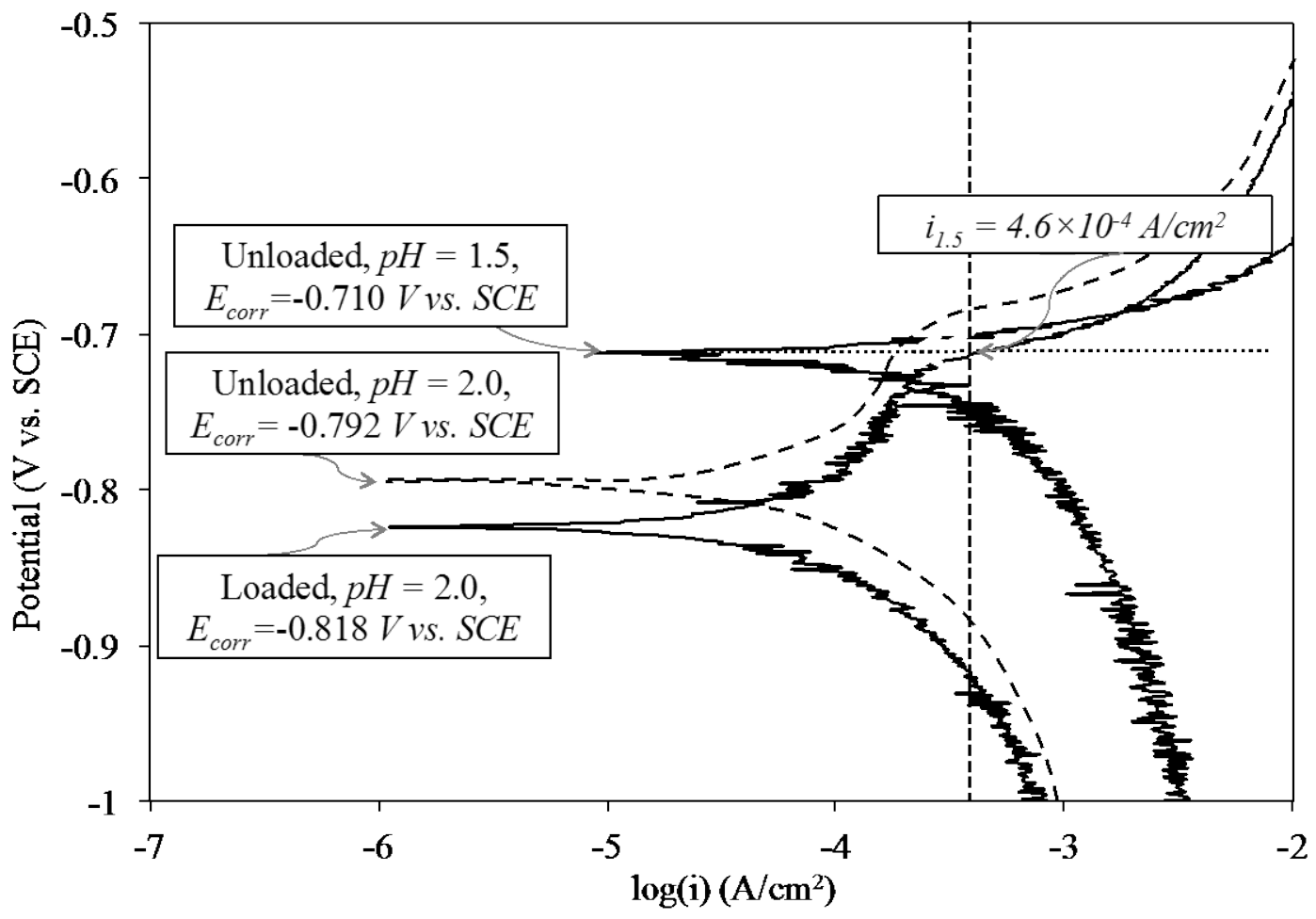

Figure 60: Predicted polarization behavior at a stress corrosion crack tip for AA6005A-T6 exposed to $3.5 \% \mathrm{NaCl}$ at $\mathrm{pH}=1.50$. 
Now consider the electrochemical reactions occurring at a stress corrosion crack tip in a loaded CT sample of AA6005A-T6 that is exposed to a solution of 3.5\% $\mathrm{NaCl}$ at $\mathrm{pH}=2.0$ (Figure 61). In this case, the cathodic reaction at the free surface should be occurring at approximately $p H=2.0$, and the anodic reaction at the crack tip should be occurring at approximately $p H=3.0$, according to the observed $L v s$. $p H$ relationship (Figure 34). The anodic reaction occurring in the crack tip solution would be shifted downward by $\sim 0.026 V v s$. SCE due to the local stress state at the crack tip, and the cathodic reaction would follow the polarization curve for an unstressed sample exposed to a $p H=2.0$ solution. Since the majority of the sample is in an unstressed state, the anodic reaction at the crack tip should be occurring at a potential approximately equal to the value of $E_{\text {Corr }}$ for the bulk sample (-0.792 Vvs. $S C E$ ). In this case, the corrosion current density at the crack tip, $i_{2.0}$, would be approximately $3.9 \times 10^{-5} \mathrm{~A} / \mathrm{cm}^{2}$. This predicted value of corrosion current density for a loaded CT sample of AA6005A-T6 exposed to a $p H=2.0$ solution is $11.8 \mathrm{X}$ lower than the predicted corrosion current density for a sample exposed to a $p H=1.5$ solution. Results from these analyses are summarized in Table 8 . 


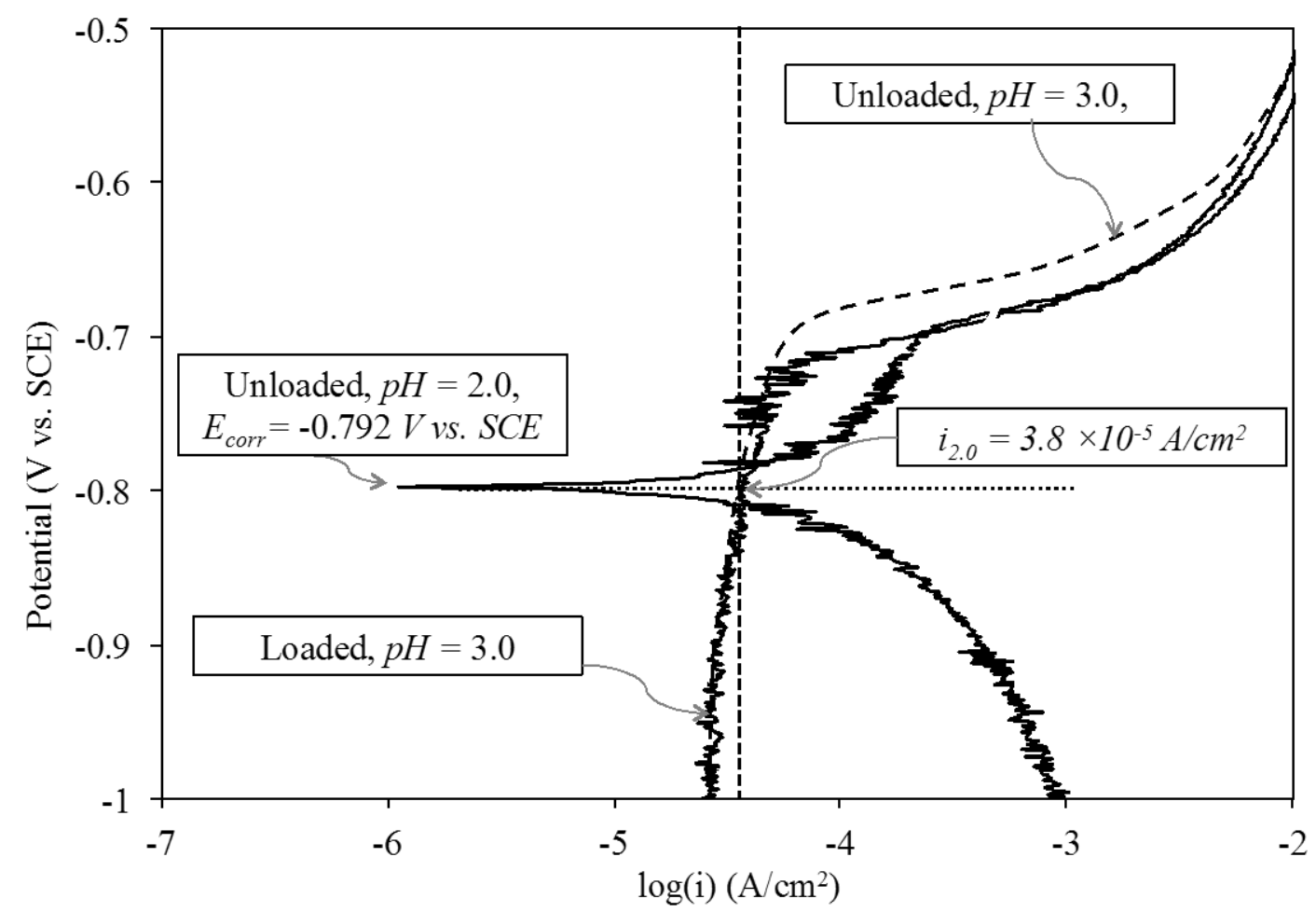

Figure 61: Predicted polarization behavior at a stress corrosion crack tip for AA6005A-T6 exposed to $3.5 \% \mathrm{NaCl}$ at $\mathrm{pH}=2.0$.

Table 8: Summary of electrochemical analysis for CT specimens exposed to $\mathrm{pH}=1.5$, and $\mathrm{pH}=2.0$ aerated saltwater solutions with and without an externally applied load.

\begin{tabular}{|c|c|c|}
\hline Exposure Conditions & $\begin{array}{c}\text { Current Density at } \\
\text { Crack Tip, } \boldsymbol{i}\end{array}$ & $\begin{array}{c}\text { Change in Current } \\
\text { Density at Crack tip }\end{array}$ \\
\hline$p H=2.0$, Applied Load & $3.9 \times 10^{-5} \mathrm{~A} / \mathrm{cm}^{2}$ & - \\
\hline$p H=1.5$, Applied Load & $4.6 \times 10^{-4} \mathrm{~A} / \mathrm{cm}^{2}$ & $+11.8 \mathrm{X}$ \\
\hline
\end{tabular}

Additional SCC-CGR experiments were conducted on CT samples of AA6005A-T6 exposed to $3.5 \% \mathrm{NaCl}$ at $\mathrm{pH}=2.0$ to examine the combined effects of an applied stress and a $p H$ gradient between the crack tip and the bulk solution on the SC crack velocity of the alloy. All experimental methods were identical to those described in section 4.5.1. Since corrosion current density is a direct measure of 
dissolution rate, the crack observed velocity in should decrease by approximately $11.8 \mathrm{X}$ when the $p H$ of the bulk solution is increased from 1.5 to 2.0 if stress-enhanced anodic dissolution is the primary mechanism of SCC in AA6005A-T6.

Figure 62 illustrates observed SC crack velocity at varied crack lengths for samples of AA6005A-T6 tested in solutions of 3.5\% $\mathrm{NaCl}$ at $\mathrm{pH}=1.5(\diamond)$ and $p H=2.0(\diamond)$. The shaded grey region in Figure 62 represents the range of observed crack velocities for samples tested in a $p H=2.0$ solution. Similar to samples tested in a $p H=1.5$ solution, the crack velocity for samples tested in a $p H=2.0$ solution did not appear to depend on crack length or applied stress intensity. However, the nominal crack velocity of samples tested in solutions at $p H=2.0$ was approximately 10.5X smaller than that of samples tested at $p H=1.5$. This observed decrease in SCC velocity at the higher solution $p H$ is consistent with the predicted decrease in SCC velocity per the analyses discussed above. Therefore it appears that SCC velocity in AA6005A-T6 exposed to low- $p H$ saltwater solutions is ultimately controlled by stress-enhanced dissolution at the crack tip. 


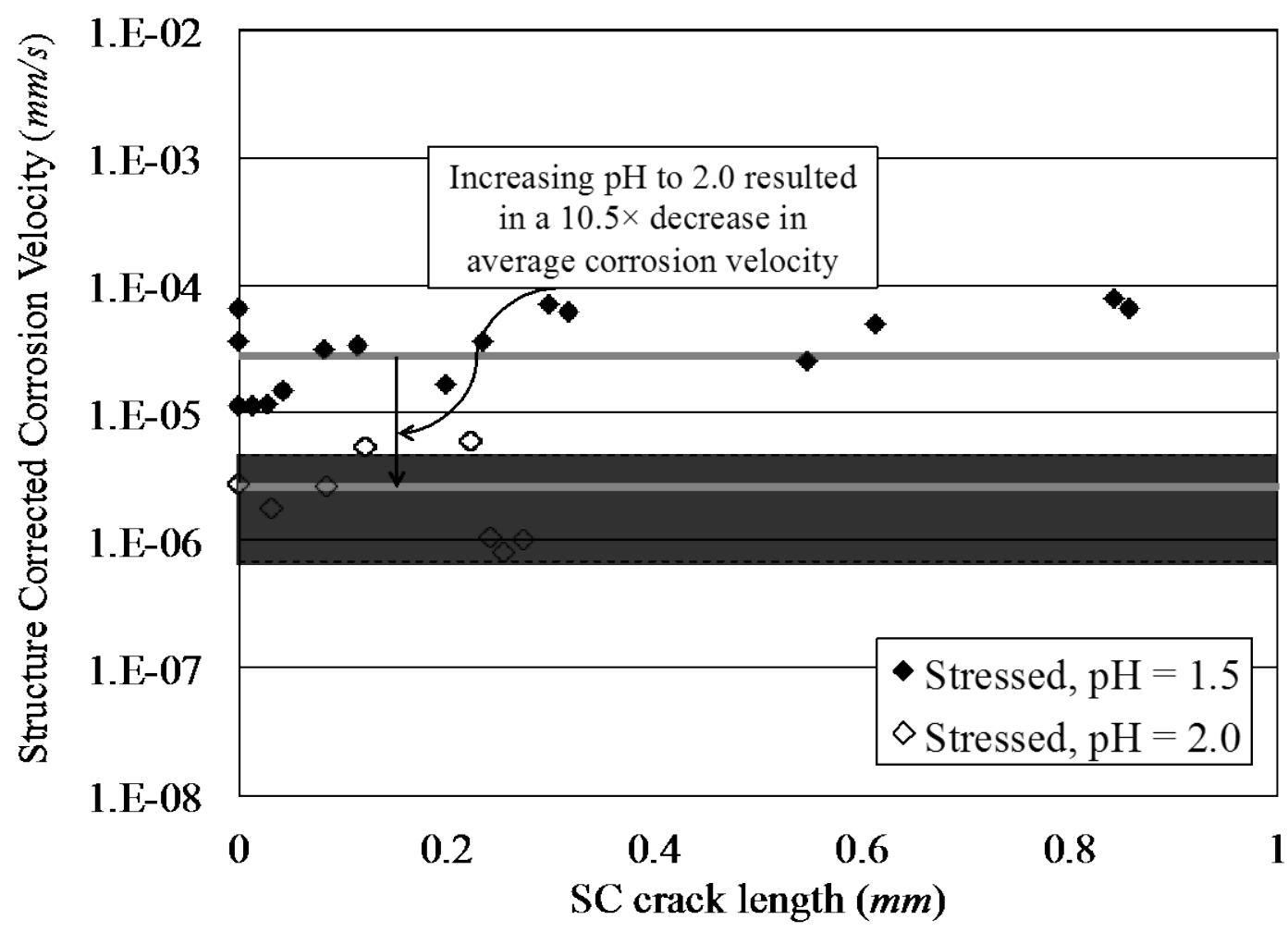

Figure 62: Structure corrected corrosion velocity for loaded CT samples of AA6005A-T6 exposed to $3.5 \% \mathrm{NaCl}$ at $\mathrm{pH}=1.5$ or $\mathrm{pH}=2.0$. The observed $10.5 \mathrm{X}$ decrease in corrosion velocity associated with increasing the $\mathrm{pH}$ of the electrolyte from 1.5 to 2.0 is consistent with the predicted decrease in crack tip current density. 


\section{Summary}

The original intent for the research presented in this this dissertation was to determine if IGC or SCC should be primary design considerations when building solar power frames of AA6005A extrusions in industrial marine environments. The effects of heat treatment and environment on the IGC susceptibility of the alloy were examined by conducting immersion tests in low- $\mathrm{pH}$ solutions of $3.5 \% \mathrm{NaCl}$. The elongated grain structure located in the core of the extrusions was of primary interest when conducting immersion tests, as factors influencing IGC susceptibility in the equiaxed grain structure near the extrusion surfaces have already been well documented. The effects of heat treatment and environment on SCC susceptibility of AA6005A were examined by performing breaking load tests (BLT) and crack growth rate (SCC-CGR) measurements on the alloy.

Potentiostatic polarization measurements were performed on samples of AA6005A in various environments to understand the effects of heat treatment and environment on the electrochemical reactions governing the IGC and SCC behaviors of AA6005A extrusions. Post-extrusion heat treatment was found to have a negligible effect on the bulk polarization behavior of the material, indicating that the anodic and cathodic reactions involved in the overall corrosion reaction for AA6005A do not depend on the heat treatment (Figure 17). The corrosion potential of AA6005A varied with $\mathrm{pH}$ when tested in a solution containing 3.5\% $\mathrm{NaCl}$ (Figure 14). The large downward shift in corrosion potential when the $p H$ of the solution was increased to 
3.0 was attributed to the passive $\mathrm{Al}_{2} \mathrm{O}_{3}$ film being stable at higher values of solution $p H(\geq 3.0)$. The corrosion current density (corrosion rate) also depended on solution $p H$ (Figure 16). A near linear decrease in current density was observed when the solution $p H$ was raised from 1.2 to 2.0 . The corrosion current density remained relatively constant at $5.3 \times 10^{-5} \mathrm{~A} / \mathrm{cm}^{2}$ when $2.0<p H<5.50$. The results from the polarization measurements served as a baseline for understanding the IGC and SCC behaviors of AA6005A extrusions.

The mode of corrosion attack in the elongated grain structure varied with heat treatment (Figure 19). IGC was observed when the alloy was in naturally aged (T4) or peak aged (T6) tempers. Local pitting became the primary mode of attack when the alloy was in an overaged (OA) condition. Similar to the equiaxed grain structure, the mode of corrosion attack in the elongated grain structure depended primarily on the morphology of $\mathrm{Cu}$-containing grain boundary precipitates (Figures 20-22). When the $\mathrm{Cu}$-rich precipitates formed a continuous (or semi-continuous) film along the grain boundaries, the alloy was susceptible to IGC.

Anisotropic IGC was observed in the elongated grain structure of AA6005A with IGC penetration being most rapid along the extrusion direction (1) and least rapid along the through-thickness direction (2) (Figures 24-26). A simple threedimensional model was developed which related the tortuosity of an IGC path along a specific direction to geometric anisotropy of the elongated grain structure (Figure 28). This model was tested by comparing the "structure-corrected" corrosion path lengths along each direction for samples exposed to a low- $p H$ saltwater solution for specific 
intervals of time. The calculated structure corrected corrosion path length agreed reasonably well along all three orthogonal directions at the specific exposure times, thus it was determined that the anisotropic IGC in the elongated grain structure was primarily due to geometric elongation of the core grain structure.

The velocity of IGC penetration in the case of unstressed samples decreased with increasing corrosion path length (Figure 30). Characterization of the local environment at the tip of a simulated corrosion path was performed to help understand the reasons for this observed decay in IGC velocity. The $p H$ at the tip of a simulated corrosion path reached an equilibrium value of $\sim 2.5$ as the corrosion path length approached $2.3 \mathrm{~mm}$ when the $\mathrm{pH}$ of the bulk solution was held at 1.5 (Figure 34). The $p H$ in the simulated corrosion path was postulated to depend on equilibrium between $\mathrm{Al}^{3+}$ created by the anodic reaction at the tip of the corrosion path and some solid precipitate species (such as $\mathrm{Al}_{2} \mathrm{O}_{3}$ or $\mathrm{Al}(\mathrm{OH})_{3}$ ) existing in the corrosion path. A model was developed that predicted IGC velocity based on one dimensional diffusion of $A l^{3+}$ from the tip of the corrosion path to the bulk environment assuming $\left[A l^{3+}\right]$ at the tip of a corrosion path is controlled exclusively by equilibrium between $A l^{3+}$ and some solid precipitate species in the corrosion path. This model seemed to accurately predict the decay in IGC velocity AA6005A-T6 with increasing corrosion path length (Figure 36), suggesting that the velocity of IGC in this alloy is ultimately limited by diffusion of $\mathrm{Al}^{3+}$ away from the tip of the IGC path.

The effects of heat treatment and environment on SCC susceptibility in AA6005A extrusions were qualitatively examined by performing breaking load tests 
(BLT). The alloy was immune to SCC when in a T4 temper and exposed to a solution of $3.5 \% \mathrm{NaCl}$ at $p H=1.0$. The alloy was only mildly susceptible to SCC when in a T6 or OA temper and exposed to the same environment (Figure 40). Adding a hydrogen recombination poison (HRP, which magnifies the effects of hydrogen embrittlement) to the test solution actually improved the residual strength of samples tested in a T6 temper (Figure 42), suggesting hydrogen embrittlement is not the primary mechanism of SCC in the alloy.

Crack growth rate (CGR) measurements were performed on compact tensile (CT) samples of AA6005A-T6 to more quantitatively examine SCC in the alloy. The CGR rate was observed to nearly constant $\left(\sim 3 \times 10^{-5} \mathrm{~mm} / \mathrm{s}\right)$ over a wide range of stress intensities $\left(9.5 \mathrm{MPa} \sqrt{ } \mathrm{m}<K_{I}<28 \mathrm{MPa} \sqrt{ } \mathrm{m}\right)$ and SC crack lengths $\left(0 \mathrm{~mm}<a_{S C C}<\right.$ $4.9 \mathrm{~mm}$ ) when exposed to an aerated solution of $3.5 \% \mathrm{NaCl}$ at $\mathrm{pH}=1.5$ (Figure 47). Adding an HRP to the test solution did not significantly change the SCC velocity, nor did it change the appearance of the fracture surface, further indicating that hydrogen embrittlement is not the primary mechanism of SCC in AA6005A (Figures 51-52).

The diffusion-based model, which was used to describe the decay in IGC velocity with increasing corrosion path length, was employed to determine if SCC velocity in AA6005A-T6 is controlled by diffusion of $A l^{3+}$ away from the crack tip. The diffusion-based model predicted the SCC velocity at the mid-plane of a CT sample would be $\sim 4 \times 10^{-7} \mathrm{~mm} / \mathrm{s}(\sim 100 \mathrm{X}$ less than the observed SCC velocity), and that SCC velocity would be greatest along the edges of the sample (which was not consistent with the observed crack tunneling). Since the diffusion-based model did 
not adequately explain the steady-state SCC velocity, it was concluded that SCC of AA6005A-T6 is not limited by diffusion of $A l^{3+}$ from the crack tip.

The stress state and electrochemical reactions near a crack tip in CT samples of AA6005A-T6 were evaluated to understand the mechanisms of SC crack growth in the alloy. Metallographic examination along the 3-2 plane of AA6005A-T6 CT samples that had been loaded along the 3 direction and exposed to the test solution revealed that there was negligible deformation along the 2 direction of the sample. This result indicated that there might be regions near the mid-plane of the CT specimens that experience a good degree of stress triaxiality. With this knowledge, the maximum stress state at the crack tip was estimated by applying the plastic constraint factor, and was determined to be approximately $750 \mathrm{MPa}$. The NabarroHerring model was then applied to determine the change in chemical potential at the crack tip due to an applied load. A small $(-0.026 \mathrm{~V})$ shift in the anodic half-cell electrochemical potential due to an applied load was predicted using Faraday's law.

The effect of a $p H$ gradient between the crack tip and the bulk aqueous environment was also considered in understanding the electrochemical reactions at the crack tip in the case of loaded CT samples of A6005A-T6 exposed to aerated saltwater at $p H=1.5$. When the bulk solution was at $p H=1.5$, the $p H$ along the centerline of the CT samples was predicted to be 2.0 based on the observed relationship between corrosion path length and $p H$ (Figure 34). In this case, the surface area of material exposed to a solution with $p H=2.0$ was small relative to the surface area of material exposed to solution with $p H=1.5$. It was thus assumed that 
the cathodic reaction governing the corrosion reaction at the crack tip would correspond to that of the bulk material, which is exposed to $p H=1.50$ solution, and that the anodic reaction at the crack tip would follow the anodic polarization behavior of AA6005A-T6 exposed to a solution with bulk $p H=2.0$. Since the majority of the specimen would be exposed to a solution with $p H=1.5$, it was assumed that both the anodic (occurring at the crack tip) and cathodic (occurring on the free surfaces of the sample) would be occurring at a potential approximately equal to the measured value of $E_{C o r r}$ for a sample exposed to a solution at $p H=1.5(0.71 V$ vs. $S C E)$.

The local electrochemical reactions at the crack tip in loaded CT samples exposed to a solution at $p H=1.5$ were evaluated by examining the polarization curves for samples exposed to solutions of $3.5 \% \mathrm{NaCl}$ at $\mathrm{pH}=1.5$ and $\mathrm{pH}=2.0$ (Figure 61). Assuming that the anodic reaction at the crack tip followed the polarization behavior of a sample exposed to an aerated saltwater solution at $p H=$ 2.0, and occurred at $\sim 0.71 V v s . S C E$, the resulting corrosion current density at the crack tip with an applied stress was estimated to be $4.6 \times 10^{-4} \mathrm{~A} / \mathrm{cm}^{2}$ when the samples were exposed to a solution with a bulk $p H$ of 1.5 . Increasing the bulk solution $p H$ to 2.0 created a situation where the both the anodic reaction would occur at $p H \approx 3.0$, and the cathodic reaction would occur at $p H=2.0$. The corrosion current density in the case of stressed samples exposed to an aerated saltwater solution with bulk $p H=$ 2.0 was estimated to be $3.9 \times 10^{-5} \mathrm{~A} / \mathrm{cm}^{2}$, which corresponded to an $11.8 \mathrm{X}$ decrease in crack tip corrosion current density. Further SCC-CGR experiments were conducted in a solution of $3.5 \% \mathrm{NaCl}$ at $\mathrm{pH}=2.0$, and the resulting $\mathrm{SCC}$ velocity was found to be 
$\sim 2.6 \times 10^{-5} \mathrm{~mm} / \mathrm{s}$ - approximately $10.5 \mathrm{X}$ smaller than the observed SCC velocity when the samples were tested in a solution at $p H=1.5$. This result was in reasonable agreement with the predicted decrease in SCC velocity mentioned above. This result suggested that SCC velocity in AA6005A-T6 is most likely controlled by the combined effects of an applied load and a $p H$ gradient between the crack tip solution and bulk solution when the alloy is exposed to a low- $p H(<2.0)$ saltwater solution. This result also further validated the argument that SCC in AA6005A-T6 is most consistent with an anodic dissolution mechanism. 


\section{Conclusions}

The IGC and SCC behavior of AA6005A was studied by examining two unique hypotheses. These hypotheses were designed evaluate the relationship between IGC and SCC susceptibility in AA6005A, and to clarify the underlying mechanisms of SCC in AA6005A. As with many research programs, many questions

arose while examining the two primary hypotheses. Though many of the unanswered questions were beyond the scope of this dissertation, several were examined further giving some valuable insight into the corrosion behavior of AA6005A. These conclusions are summarized below in the section entitled "Additional Conclusions".

\subsection{Conclusions}

\subsubsection{Conclusions based on Primary Hypotheses}

Hypothesis 1: Metallurgical and environmental conditions, which promote IGC susceptibility in AA6005A extrusions, will also promote SCC susceptibility. Result: IGC testing of AA6005A extrusions revealed that the elongated grain structure was susceptible to IGC when in a naturally aged (T4) temper or peak aged (T6) temper when exposed to an aerated solution of $3.5 \% \mathrm{NaCl} p H<2.0$. Pitting was observed when the material was in an overaged (OA) temper in these environments (Figure 19). Results from qualitative SCC testing of AA6005A extrusions demonstrated that the material was immune to SCC when in a T4 condition at $p H<2.0$, and only mildly susceptible to SCC when in a T6 or OA 
condition when $p H<2.0$ (Figure 40). These results suggest that Hypothesis 1 is false.

Hypothesis 2: The mechanism of SCC in AA6005A extrusions is enhanced anodic dissolution at the crack tip due to applied stress.

Result: Adding a hydrogen recombination poison to test solutions improved the residual strength of AA6005A-T6 in qualitative SCC testing (Figure 42). In contrast, the addition of a hydrogen recombination poison had a negligible effect on the SC crack velocity in quantitative SCC testing (Figure 51). Though these results are conflicting, it can be concluded that changes in the hydrogen discharge reactions to promote hydrogen embrittlement do not degrade the SCC resistance of the material. Therefore it does not appear that hydrogen embrittlement is a primary mechanism of SCC in AA6005A-T6 exposed to acidified saltwater solutions. The observed crack velocity in quantitative SCC testing was consistent with enhanced dissolution due to the combined effects of an applied stress and a $p H$ gradient within the cracks (Figures 60-62). These results suggest an anodic dissolution mechanism best explains SCC in AA6005A, confirming Hypothesis 2.

\subsubsection{Additional Conclusions}

- The corrosion potential in AA6005A tested in 3.5\% $\mathrm{NaCl}$ varies with the $\mathrm{pH}$ of the solution (Figure 14). A large increase in corrosion potential when $p H<$ 3.00 is due to the rapid dissolution of the passive layer at low $p H$. Though the passive layer is thermodynamically unstable when $p H<4.00$, it dissolves 
very slowly when $p H>2.00$, and the experimentally measured polarization behavior corresponds to polarization of the passive layer

- The mode of corrosion attack in the elongated grain structure of AA6005A extrusions varies with heat treatment, and depends primarily on the morphology of $\mathrm{Cu}$-containing grain boundary precipitates, which are noble relative to $A l$ (Figures 20-22). Though the EDS analysis was qualitative, the composition of the grain boundary precipitates in the elongated grain structure seems to be consistent with $Q$-phase precipitates, as observed by the Nisancioglu group [4-8]. The $\mathrm{Cu}$-rich structure formed a continuous film along grain boundaries when the material was in a $\mathrm{T} 4$ or $\mathrm{T} 6$ condition, and IGC was observed to be the dominant mode of attack in these heat treatments. The $\mathrm{Cu}$-rich phase coarsened to form discrete particles within an OA age treatment. Pitting was the dominant mode of attack in samples tested in an OA temper.

- IGC in the elongated grain structure of AA6005A-T4 and AA6005A-T6 extrusions is highly anisotropic, with IGC propagating most rapidly along the extrusion direction, and least rapidly along the through-thickness direction (Figures 24-26). A simple three-dimensional model, which related grain dimensions along different directions to the tortuosity of an IGC path was developed (Figure 28), and it was determined that the observed anisotropy of 
IGC in the elongated grain structure of AA6005A-T4 and AA6005A-T6 extrusions was simply due to elongation of the grains.

- The rate of IGC penetration in the case of unloaded samples of AA6005A-T6 is limited by the diffusion of $A l^{3+}$ away from the tip of the IGC path. At small corrosion path lengths, there is a large gradient in $\left[\mathrm{Al}^{3+}\right]$ between the tip of the IGC path and the external environment resulting in rapid diffusion of $A l^{3+}$ away from the tip of the IGC path, and rapid IGC penetration. As the corrosion path length increases, the gradient in $\left[A l^{3+}\right]$ becomes less, slowing diffusion of $\mathrm{Al}^{3+}$ away from the tip of the IGC path, therefore slowing the rate of IGC penetration (Figure 36).

- Qualitative SCC testing of AA6005A extrusions demonstrated that the material is only mildly susceptible to SCC when in a T6 or OA condition, and exposed to an aggressive $(1.00<p H<2.00)$ acidified saltwater environment. The material was immune to SCC when in a T4 condition (Figure 40). $\underline{\text { These }}$ results indicate that AA6005A extrusions will likely be able to withstand the proposed design loads and lifespan for a CSPP frame (See section 1).

- $\quad$ Results from SCC-CGR revealed that crack growth rates in AA6005A-T6 exposed to aerated saltwater at $p H=1.5$ were constant at approximately $3 \times 10^{-}$ ${ }^{5} \mathrm{~mm} / \mathrm{s}$ over a wide range of stress intensities $\left(9 \mathrm{MPa} \sqrt{\mathrm{m}}<K_{I}<28 \mathrm{MPa} \sqrt{\mathrm{m}}\right)$ 
and crack lengths (Figure 47). Increasing the $p H$ of the solution to 2.0 caused a 10.5X decrease in crack velocity (Figure 61). The observed decrease in SCC-CGR velocity is consistent with what would be expected if the crack velocity was determined by the combined effects stress and a $\mathrm{pH}$ gradient between the crack tip solution and the bulk solution (Figure 60). When the bulk solution is at $p H=1.5$, the $p H$ near the crack tip would be approximately 2.0. In this case, the cathodic reaction would be occurring at $p H=1.5$, and the anodic reaction governing the crack growth would occur at $p H=2.0$, which yields a predicted current density at the crack tip of $4.6 \times 10^{-4} \mathrm{~A} / \mathrm{cm}^{2}$. By increasing the bulk solution $p H$ to 2.0 , the anodic reaction controlling SCC velocity would be occurring at approximately $p H=3.0$, while the cathodic reaction would be occurring at $p H=2.0$. In this case, the current density at the crack tip was estimated to be $\sim 3.9 \times 10^{-5} \mathrm{~A} / \mathrm{cm}^{2}$. The $11.8 \mathrm{X}$ decrease in predicted current density associated with increasing the bulk solution $p H$ from 1.5 to 2.0 is consistent with what was observed in the SCC-CGR experiments. 


\section{References}

1. Birol, Y., DSC Analysis of the precipitation reaction in AA6005 alloy. Journal of Thermal Analysis and Calorimetry, 2008. 93(3): p. 977-981.

2. $\quad$ Eskin, D.G., V. Massardier, and P. Merle, A study of high-temperature precipitation in Al-Mg-Si alloys with an excess of silicon. Journal of Materials Science, 1999. 34(4): p. 811-820.

3. Jacobs, M.H., The structure of the metastable precipitates formed during ageing of an Al-Mg-Si alloy. Philosophical Magazine, 1972. 26(1): p. 1-13.

4. Larsen, M.H. et al., Intergranular Corrosion of Copper-Containing AA6xxx AlMgSi Aluminum Alloys. Journal of the Electrochemical Society, 2008. 155(11): p. C550-C556.

5. Svenningsen, G., et al., Effect of high temperature heat treatment on intergranular corrosion of AlMgSi(Cu) model alloy. Corrosion Science, 2006. 48(1): p. 258-272.

6. Svenningsen, G., et al., Effect of thermomechanical history on intergranular corrosion of extruded AlMgSi(Cu) model alloy. Corrosion Science, 2006. 48(12): p. 3969-3987.

7. Svenningsen, G., et al., Effect of artificial aging on intergranular corrosion of extruded AlMgSi alloy with small Cu content. Corrosion Science, 2006. 48(6): p. 1528-1543.

8. Svenningsen, G., et al., Effect of low copper content and heat treatment on intergranular corrosion of model AlMgSi alloys. Corrosion Science, 2006. 48(1): p. 226-242.

9. Minoda, T. and H. Yoshida, Effect of grain boundary characteristics on intergranular corrosion resistance of 6061 aluminum alloy extrusion. Metallurgical and Materials Transactions A, 2002. 33(9): p. 2891-2898.

10. Birol, Y., Impact of partial recrystallization on the performance of $6005 \mathrm{~A}$ tube extrusions. Engineering Failure Analysis, 2010. 17(5): p. 1110-1116.

11. Bryant, A.J., Development of directional microstructures in mechanical processing of aluminium alloys. Metals Technology, 1975. 2(1): p. 21-32.

12. Knight, S.P., et al., Understanding the Directional Dependence of Intergranular Corrosion in Aluminium Alloys. Materials Science Forum. 654656: p. 946-949.

13. Liu, X., et al., Effect of applied tensile stress on intergranular corrosion of AA2024-T3. Corrosion Science, 2004. 46(2): p. 405-425.

14. Puiggali, M., et al., Effect of microstructure on stress corrosion cracking of an Al-Zn-Mg-Cu alloy. Corrosion Science. 40(4-5): p. 805-819.

15. Zhang, W., et al., Statistical model for intergranular corrosion growth kinetics. Corrosion Science, 2003. 45(2): p. 353-370.

16. Zhao, S., et al., Generalized model for IGC growth in aluminum alloys. Journal of Statistical Planning and Inference, 2007. 137(7): p. 2405-2412. 
17. Zhang, W. and G.S. Frankel, Transitions between pitting and intergranular corrosion in AA2024. Electrochimica Acta, 2003. 48(9): p. 1193-1210.

18. Stansbury, E.E., Buchanan, R.A., Fundamentals of Electrochemical Corrosion. 2000, Materials Park, OH: ASM International.

19. Jones, D.A., Principles and Prevention of Corrosion. 2 ed. 1996, Upper Saddle River, NJ: Prentice Hall. 572.

20. Gao, L.J. and B.E. Conway, Poisoning effects of arsenic species on $H$ adsorption and kinetic behaviour of the $\mathrm{H} 2$ evolution reaction at $\mathrm{Pt}$ in $\mathrm{KOH}$ solution. Journal of Electroanalytical Chemistry, 1995. 395(1-2): p. 261-271.

21. Qian, S.Y., B.E. Conway, and G. Jerkiewicz, Kinetic rationalization of catalyst poison effects on cathodic H sorption into metals: relation of enhancement and inhibition to H coverage. Journal of the Chemical Society, Faraday Transactions, 1998. 94(19): p. 2945-2954.

22. Hunter, M.S., Fowle, P., Naturally and Thermally Formed Oxide Films on Aluminum. Journal of the Electrochemical Society, 1956. 103.

23. Foley, R.T., Localized Corrosion of Aluminum Alloys - A Review. Corrosion, 1986. 42: p. 277-288.

24. Kaesche, H. Pitting Corrosion of Aluminum and Intergranular Corrosion of Aluminum Alloys. in Localized Corrosion NACE 3. 1974.

25. Vargel, C., Corrosion of Aluminum. Vol. 1. 2004: Elsevier.

26. Vargel, C., in Corrosion of Aluminum. 2004, Elsevier. p. 122.

27. Jones, R.H., Stress Corrosion Cracking, in ASM Handbook. 2003, ASM International. p. 346-366.

28. Ghali, E., in Corrosion Resistance of Aluminum and Magnesium Alloys. 2010, Wiley. p. 289.

29. Vargel, C., in Corrosion of Aluminum. 2004, Elsevier. p. 128.

30. Burleigh, T.D., The Postulated Mechanisms for Stress Corrosion Cracking of Aluminum Alloys - A Review of the Literature 1980-1989. Corrosion Science, 1990. 47(2).

31. Speidel, M.O., Stress Corrosion Cracking of Aluminum Alloys. Metallurgical Transactions A, 1975. 6A.

32. Bond, G.M., I.M. Robertson, and H.K. Birnbaum, Effects of hydrogen on deformation and fracture processes in high-purity aluminium. Acta Metallurgica, 1988. 36(8): p. 2193-2197.

33. Vehoff, H. and H.K. Klameth, Hydrogen embrittlement and trapping at crack tips in Ni-single crystals. Acta Metallurgica, 1985. 33(6): p. 955-962.

34. Vehoff, H. and W. Rothe, Gaseous hydrogen embrittlement in FeSi-and Nisingle crystals. Acta Metallurgica, 1983. 31(11): p. 1781-1793.

35. Vogt, H. and M.O. Speidel, Stress corrosion cracking of two aluminium alloys: A comparison between experimental observations and data based on modelling. Corrosion Science, 1998. 40(2-3): p. 251-270.

36. Olden, V., et al., Cohesive zone modeling of hydrogen-induced stress cracking in 25\% Cr duplex stainless steel. Scripta Materialia, 2007. 57(7): p. 615-618. 
37. Shim, I.O. and J.G. Byrne, A study of hydrogen embrittlement in 4340 steel I: Mechanical aspects. Materials Science and Engineering: A, 1990. 123(2): p. 169-180.

38. A.J. Sedriks, J.A.S.G., D.L. Novak, Corrosion Processes and Solution Chemistry within Stress Corrosion Cracks in Aluminum Alloys, in NACE-3. 1974. p. 569-575.

39. Davis, J.A. $p H$ and Potential Measurements During Stress Corrosion of Aluminum Alloys. in AGARD. 1972. London: AGARD.

40. Davis, J.A., Use of Microelectrode for Study of Stress Corrosion of Aluminum Alloys, in NACE-3. 1974. p. 168-172.

41. Sedriks, A.J., J.A.S. Green, and D.L. Novak, Comparison of the corrosion and stress-corrosion behavior of a ternary Al-Zn-Mg alloy. Metallurgical Transactions, 1970. 1(7): p. 1815-1819.

42. R. Piccinini, M.M., A.J.E. Pourbaix, R.F. Hochman, A Study of Simluated Stress Corrosion Crack Tip Electrochemical Reactions, in NACE-3. 1974. p. 179-183.

43. Edeleanu, C.E., U.R., The causes of the localized character of corrosion on aluminum. Transactions of the Faraday Society, 1951. 47: p. 1121-1135.

44. Sedriks, A., J.A. Green, and D. Novak, On the Chemistry of the Solution at the Tips of Stress-Corrosion Cracks in Aluminum Alloys. 1970, DTIC Document.

45. Cooper, K.R., Young, L.M., Gangloff, R.P., Kelly, R.G., The Electrode Potential Dependence of Environment-Assisted Cracking of AA7050. Materials Science Forum, 2000. 311-337: p. 1625-1634.

46. Liu, X., et al., In-situ observation of intergranular stress corrosion cracking in AA2024-T3 under constant load conditions. Corrosion Science, 2007. 49(1): p. 139-148.

47. Dymek, S. and M. Dollar, TEM investigation of age-hardenable Al 2519 alloy subjected to stress corrosion cracking tests. Materials Chemistry and Physics, 2003. 81(2-3): p. 286-288.

48. DeWeese, S.K., Ningileri, S.T., Das, S.K., Green, J.A.S., Stress-CorrosionCracking in Aluminum Beverage Can Ends-Issues, Obersvations, and Potential Solutions. Developments in Aluminum, 2008: p. 50-57.

49. Onoro, J., Ranninger, C., Stress-Corrosion-Cracking Behavior of HeatTreated Al-Zn-Mg-Cu Alloy With Temperature. Materials Science, 1999. 35(4): p. 509-514.

50. Scamans, G.M., N.J.H. Holroyd, and C.D.S. Tuck, The role of magnesium segregation in the intergranular stress corrosion cracking of aluminium alloys. Corrosion Science, 1987. 27(4): p. 329-347.

51. Searles, J.L., Gouma, P.I., Buchheit, R.G., Stress Corrosion Cracking of Sensitized AA5083 (Al-4.5Mg-1,0Mn). Metallurgical and Materials Transactions A, 2001. 32A.

52. Guillaumin, V. and G. Mankowski, Localized corrosion of 6056 T6 aluminium alloy in chloride media. Corrosion Science, 2000. 42(1): p. $105-$ 125. 
53. Kciuk, M., The structure, mechanical properties and corrosion resistance of aluminum $\mathrm{AlMg}_{1} \mathrm{Si}_{1}$ alloy. Journal of Achievments in Materials and Manufacturing Engineering, 2006. 16(1-2): p. 51-56.

54. Liu, Y., et al., Precipitation in an AA6111 aluminium alloy and cosmetic corrosion. Acta Materialia, 2007. 55(1): p. 353-360.

55. Gruhl, W., Stress-Corrosion Cracking of High Strength Aluminum Alloys. Zeitschrift Fur Metallkunde, 1984. 75(11): p. 819-826.

56. Izu, M., Shiokawa, M., Sato, E., Effect of stress and applied current density on stress corrosion cracking of 2017 aluminum alloy. Journal of Japan Institute of Light Metals, 1984. 34(11): p. 643-649.

57. Izumoto, E. and R. Nishimura, Environment-induced cracking of Al-Cu alloy (AA2017P-T3) under constant load in distilled water and sodium chloride solutions. Corrosion Science. 53(3): p. 886-893.

58. Kramer, L., et al., Stress-corrosion cracking susceptibility of various product forms of aluminum alloy 2519. Journal of Materials Engineering and Performance, 2002. 11(6): p. 645-650.

59. Albrecht, J., A.W. Thompson, and I.M. Bernstein, The role of microstructure in hydrogen-assisted fracture of 7075 aluminum. Metallurgical Transactions A, 1979. 10(11): p. 1759-1766.

60. Hardwick, D.A., A.W. Thompson, and I.M. Bernstein, The effect of copper content and microstructure on the hydrogen embrittlement of AI-6Zn-2Mg alloys. Metallurgical Transactions A, 1983. 14(12): p. 2517-2526.

61. Young, G., Jr. and J. Scully, The effects of test temperature, temper, and alloyed copper on the hydrogen-controlled crack growth rate of an Al-Zn-Mg(Cu) alloy. Metallurgical and Materials Transactions A, 2002. 33(4): p. 11671181.

62. Knight, S.P., et al., Correlations between intergranular stress corrosion cracking, grain-boundary microchemistry, and grain-boundary electrochemistry for Al-Zn-Mg-Cu alloys. Corrosion Science. 52(12): p. 40734080.

63. Pickens, J.R., J.R. Gordon, and J.A.S. Green, THE EFFECT OF LOADING MODE ON THE STRESS-CORROSION CRACKING OF ALUMINUMALLOY 5083. Metallurgical Transactions a-Physical Metallurgy and Materials Science, 1983. 14(5): p. 925-930.

64. Furu, T., Vatne, Hans Erick., Texture Evolution during Extrusion of Flat AA6060 and AA6082 Aluminum Profiles. Materials Science Forum, 1998. 273-275: p. 403-410.

65. Cooper, K.R. and R.G. Kelly, Crack tip chemistry and electrochemistry of environmental cracks in AA 7050. Corrosion Science, 2007. 49(6): p. 26362662.

66. Boudreau, B.P., Diagnetic Models and Their Imlemntation. Vol. 1. 1997: Springer. 428.

67. Li, Y.H., Gregory, Sandra, Diffusion of Ions in Sea Water and Deep-Sea Sediments. Geochimica et Cosmochimica Acta, 1974. 38: p. 703-714. 
68. Pourbaix, M., in Atlas of Electrochemical Equilibria. 1966, Pergamon Press Ltd. p. 168-175.

69. ASTM, ASTM G139 - 05(2011) Standard Test Method for Determining Stress-Corrosion Cracking Resistance of Heat-Treatable Aluminum Alloy Products Using Breaking Load Method. 2011.

70. Berkeley, D.W., H.E.M. Sallam, and H. Nayeb-hashemi, The effect of pH on the mechanism of corrosion and stress corrosion and degradation of mechanical properties of AA6061 and Nextel 440 fiber-reinforced AA6061 composite. Corrosion Science, 1998. 40(2-3): p. 141-153.

71. Braun, R., Environmentally assisted cracking of aluminium alloys. Materialwissenschaft und Werkstofftechnik, 2007. 38(9): p. 674-689.

72. Hertzberg, R.W., Deformation and Fracture Mechanics of Engineering Materials. Vol. 3. 1989: Hamilton Printing Company.

73. Oñoro, J., The stress corrosion cracking behaviour of heat-treated Al-Zn$\mathrm{Mg}-\mathrm{Cu}$ alloy in modified salt spray fog testing. Materials and Corrosion. 61(2): p. 125-129.

74. Bovard, F., Environmentally Induced Cracking of an Al-Zn-Mg-Cu Alloy, in Materials Science and Engineering. 2005, University of Pittsburgh: Pittsburgh, PA. p. 118.

75. Broek, D., Elementary Engineering Fracture Mechanics. 1983, Martinus Nijhoff Publishers: The Netherlands. p. 96-111.

76. Herring, G., Diffusional Viscosity of a Polycrystalline Solid Journal of Applied Physics, 1950. 21: p. 437.

77. Nabarro, F.R.N., Report of a Conference on the Strength of Solids. Physical Society, London, 1948: p. 75.

78. Galvele, J., A stress corrosion cracking mechanism based on surface mobility. Corrosion Science, 1987. 27(1): p. 1-33.

79. Galvele, J.R., Surface mobility mechanism of stress-corrosion cracking. Corrosion Science, 1993. 35(1-4): p. 419-434.

80. Galvele, J.R., Recent developments in the surface-mobility stress-corrosioncracking mechanism. Electrochimica Acta, 2000. 45(21): p. 3537-3541.

81. Newman, R.C., Developments in the Slip-Dissolution Model of Stress Corrosion Cracking. Corrosion, 1994. 50(9): p. 682-686.

82. Sieradzki, K. and F.J. Friedersdorf, Notes on the surface mobility mechanism of stress-corrosion cracking. Corrosion Science, 1994. 36(4): p. 669-675.

83. Revie, R.W., Uhlig, Herbert H., Corrosion and Corrosion Control: An Introduction to Corrosion Science and Engineering. Vol. 1. 2008, Hoboken, New Jersey: John Wiley \& Sons. 


\section{Appendix A}

The values of mode I stress intensity factor $\left(K_{I}\right)$ in this dissertation were determined by conducting strain energy release rate analysis. The steps involved in calculating $K_{I}$ followed the methods of Hertzberg [72], and are summarized in this appendix.

First consider a loaded CT sample with total crack length $a$. The relationship between modulus (M, Force/displacement) and crack length was determined by constructing a calibration curve (Figure 44). The compliance, $C$, of a loaded CT sample with varying crack length was then related to the to the modulus using Equation A 1

Equation A 1

$$
C_{E}(a)=\frac{1}{M_{E}(a)}
$$

Under constant load conditions (as used in this work), the strain energy release rate $(G)$ with increasing crack length is related to the compliance of a CT sample by Equation A 2.

\section{Equation A 2}

$$
G=\frac{1}{2} F^{2} \frac{\partial C}{\partial a}
$$

Where $F$ is the applied force. At a critical load, $F_{C}$ (load necessary to advance the crack), the critical strain energy release rate, $G_{C}$ is: 
Equation A 3

$$
G_{C}=\frac{1}{2} F_{C}^{2} \frac{\partial C}{\partial a}
$$

The critical strain energy release rate is related to mode I stress intensity and Young's modulus ( $E$ ) by Equation A 4 under plane stress conditions.

Equation A 4

$$
K_{I}=\sqrt{E G_{C}}
$$




\section{Appendix B}

The value of maximum tensile stress near a crack front in a loaded CT sample of AA6005A-T6 was estimated by applying the plastic constraint factor $(P C F)$, and following the methods of Broek [75]. The $P C F$ is defined as:

\section{Equation B 1}

$$
P C F=\sigma_{\max } / \sigma_{y}
$$

Where $\sigma_{\max }$ is the maximum tensile stress near a crack tip, and $\sigma_{y}$ is the bulk yield stress of the material. The value of $\sigma_{y}$ can be calculated by applying the Von Mises yield criterion (Equation B 2).

Equation B 2

$$
2 \sigma_{y}^{2}=\left(\sigma_{1}-\sigma_{2}\right)^{2}+\left(\sigma_{2}-\sigma_{3}\right)^{2}+\left(\sigma_{3}-\sigma_{1}\right)^{2}
$$

Where $\sigma_{1}, \sigma_{2}$, and $\sigma_{3}$ are the principal stresses along directions 1,2 , and 3, respectively. The principal stresses at various points within a stress field near the crack tip can be taken as:

\section{Equation B 3}

$$
\sigma_{1}=\left(\frac{K}{\sqrt{2 \pi r}}\right) \cos \left(\frac{\theta}{2}\right)\left(1+\sin \left(\frac{\theta}{2}\right)\right)
$$

Equation B 4

$$
\sigma_{2}=\left(\frac{K}{\sqrt{2 \pi r}}\right) \cos \left(\frac{\theta}{2}\right)\left(1-\sin \left(\frac{\theta}{2}\right)\right)
$$

Equation B 5

$$
\sigma_{3}=2 v\left(\frac{K}{\sqrt{2 \pi r}}\right) \cos \left(\frac{\theta}{2}\right)
$$


Where $K$ is the applied stress intensity, $r$ is the distance from the crack tip, $\theta$ is an angular coordinate, and $v$ is Poisson's ratio. If we take $\sigma_{2}=n \sigma_{l}$ and $\sigma_{3}=m \sigma_{l}$, the Von Mises yield criterion can be rearranged as:

\section{Equation B 6}

$$
\frac{\sigma_{1}}{\sigma_{y}}=\left(1-n-m+n^{2}+m^{2}-n m\right)^{-1 / 2}
$$

From Equations B3-B5, the values of $m$ and $n$ can become:

Equation B 7

$$
n=\frac{1-\sin \left(\frac{\theta}{2}\right)}{1+\sin \left(\frac{\theta}{2}\right)}
$$

Equation B 8

$$
m=\frac{2 v}{1+\sin \left(\frac{\theta}{2}\right)}
$$

In the plane $\theta=0$ (the plane of an advancing crack), it can be determined that $m=2 v$, and $n=1$. Assuming the value of $v$ is approximately $1 / 3$, the value of PCF in the case of plain strain loading becomes approximately 3 . This means that the maximum tensile stress near a crack is approximately $3 \sigma_{y}$. 